\title{
The boundary regularity of non-linear parabolic systems II
}

\author{
Verena Bögelein ${ }^{\mathrm{a}}$, Frank Duzaar ${ }^{\mathrm{a}, *}$, Giuseppe Mingione ${ }^{\mathrm{b}}$ \\ a Department Mathematik, Universität Erlangen-Nürnberg, Bismarckstrasse 1 1/2, 91054 Erlangen, Germany \\ b Dipartimento Mathematica, Università di Parma, Parco delle Scienze 53/a, Campus, 43100 Parma, Italy
}

Received 5 March 2009; accepted 29 April 2009

Available online 29 September 2009

\begin{abstract}
This is the second part of a work aimed at establishing that for solutions to Cauchy-Dirichlet problems involving general non-linear systems of parabolic type, almost every parabolic boundary point is a Hölder continuity point for the spatial gradient of solutions. Here we establish higher fractional differentiability of solutions up to the boundary. Based on the necessary and sufficient condition for regular boundary points from the first part of Bögelein et al. (in this issue) [7] we achieve dimension estimates for the boundary singular set and eventually the almost everywhere regularity of solutions at the boundary.
\end{abstract}

๑) 2009 L'Association Publications de l'Institut Henri Poincaré. Published by Elsevier B.V. All rights reserved.

\section{Introduction and results}

In this paper we continue the study, initiated in [7], of the partial boundary regularity of solutions to the CauchyDirichlet problems for general non-linear parabolic systems with linear growth. In the first part we gave - see Theorem 1.2 below - a regularity criterion allowing to establish that a boundary point is regular, that is, the spatial gradient of the solutions is Hölder continuous in a relative neighborhood of such a point. Such a result is an essential preliminary step towards the boundary regularity, in that it gives a necessary and sufficient condition for boundary regularity, but at the same time turns out to be insufficient to prove the existence of even one regular boundary point when not combined to further qualitative properties of solutions. In this paper we indeed prove certain weak differentiability boundary properties allowing to conclude that the criterion in question is satisfied almost everywhere at the boundary completing the proof of the basic result asserting that in the case of Cauchy-Dirichlet problems involving parabolic systems with linear growth, almost every boundary point, with respect to the usual surface measure of the parabolic boundary, is regular. It is perhaps worth mentioning that before this result even the existence of one regular boundary point for solutions was an open issue for the general systems hereby considered. To measure the progress yielded by this result we recall that the existence of boundary irregular points is already known in the elliptic case [21], even for smooth boundary data; see [32] for counterexamples in the parabolic case. Full boundary regularity is only known for parabolic systems having a special structure, like the parabolic $p$-Laplacean system with zero boundary data [10,11]. Global partial regularity of the solution - not the gradient - of quasilinear systems was shown in [3].

\footnotetext{
* Corresponding author.

E-mail addresses: boegelein@mi.uni-erlangen.de (V. Bögelein), duzaar@mi.uni-erlangen.de (F. Duzaar), giuseppe.mingione@unipr.it (G. Mingione).
} 
Specifically, we shall consider Cauchy-Dirichlet problems involving non-linear parabolic systems of the following type:

$$
\begin{cases}u_{t}-\operatorname{div} a(x, t, D u)=0 & \text { in } \Omega_{T}, \\ u=g & \text { on } \partial_{\mathcal{P}} \Omega_{T},\end{cases}
$$

defined in the cylindrical domain $\Omega_{T}=\Omega \times(0, T)$ where $\Omega \subset \mathbb{R}^{n}, n \geqslant 2$, is a bounded domain in $\mathbb{R}^{n}$ and $T>0$, and under natural linear growth and ellipticity assumptions on the vector field $a: \Omega_{T} \times \mathbb{R}^{N n} \rightarrow \mathbb{R}^{N n}$, to be specified in a few lines. The boundary values are assumed - in the sense of traces - on the parabolic boundary which is defined by

$$
\partial_{\mathcal{P}} \Omega_{T}=\partial \Omega_{T} \backslash(\Omega \times\{T\}) .
$$

According to such assumptions the notion of a weak solution of (1.1) is the following:

Definition 1.1. A map $u \in L^{2}\left(0, T ; W^{1,2}\left(\Omega, \mathbb{R}^{N}\right)\right)$ is called a (weak) solution to (1.1) if and only if

$$
\int_{\Omega_{T}} u \cdot \varphi_{t}-\langle a(x, t, D u), D \varphi\rangle d z=0
$$

holds for every test-function $\varphi \in C_{0}^{\infty}\left(\Omega_{T}, \mathbb{R}^{N}\right)$, and the following boundary conditions hold:

$$
u(\cdot, t)-g(\cdot, t) \in W_{0}^{1,2}\left(\Omega ; \mathbb{R}^{N}\right) \text { for a.e. } t \in(0, T),
$$

and

$$
\lim _{h \downarrow 0} \frac{1}{h} \int_{0}^{h} \int_{\Omega}|u(x, t)-g(x, 0)|^{2} d x d t=0 .
$$

Here we assume that the vector field $a: \Omega_{T} \times \mathbb{R}^{N n} \rightarrow \mathbb{R}^{N n}$ fulfills the following growth and ellipticity assumptions: the mappings $(z, u, w) \mapsto a(z, u, w)$ and $(z, u, w) \mapsto \partial_{w} a(z, u, w)$ are continuous in $\Omega_{T} \times \mathbb{R}^{N} \times \mathbb{R}^{N n}$ and satisfy

$$
\left\{\begin{array}{l}
|a(z, w)|+(1+|w|)\left|\partial_{w} a(z, w)\right| \leqslant L(1+|w|), \\
\left\langle\partial_{w} a(z, w) \tilde{w},\left.\tilde{w}|\geqslant v| \tilde{w}\right|^{2},\right. \\
\left|a(x, t, w)-a\left(x_{0}, t, w\right)\right| \leqslant L \tilde{\theta}\left(\left|x-x_{0}\right|\right)(1+|w|), \\
\left|a(x, t, w)-a\left(x, t_{0}, w\right)\right| \leqslant L \tilde{\theta}\left(\sqrt{\left|t-t_{0}\right|}\right)(1+|w|),
\end{array}\right.
$$

for every choice of $z, z_{0} \in \Omega_{T}, x, x_{0} \in \Omega, t, t_{0} \in(0, T)$ and $w, \tilde{w} \in \mathbb{R}^{N n}$, where

$$
\tilde{\theta}(s) \leqslant \min \left\{1, s^{\beta}\right\}, \quad s>0 .
$$

The structure constants will satisfy $0<v \leqslant 1 \leqslant L<\infty$. Concerning the regularity of the lateral boundary and the Dirichlet boundary values i.e. of $\partial \Omega$ and $g$, since the point of the paper is to prove the existence of boundary regular points we may assume that the data $\partial \Omega, g$ are smooth; on the other hand we may assume an essentially optimal regularity for them. Specifically we shall assume that

$$
\partial \Omega \text { is } C^{1, \beta}, \quad D g \in C^{\beta, 0}\left(\bar{\Omega} \times[0, T) ; \mathbb{R}^{N n}\right), \quad \partial_{t} g \in L^{2,2-2 \beta}\left(\Omega_{T} ; \mathbb{R}^{N n}\right) .
$$

For the definition of parabolic Morrey spaces of the type $L^{2,2-2 \beta}$ used here we refer to [7, Definition 2.1]. In order to study the boundary regularity of solutions we recall the definition of the set of regular boundary points

$$
\operatorname{Reg}_{\mathcal{P}} u \equiv\left\{z_{0} \in \partial_{\mathcal{P}} \Omega_{T}: D u \in C^{0}\left(\overline{U \cap \Omega_{T}} ; \mathbb{R}^{N n}\right) \text { for some neighborhood } U \text { of } z_{0}\right\} .
$$

Then, the following result has been proved in [7, Theorem 1.2]:

Theorem 1.2. Let $u \in L^{2}\left(0, T ; W^{1,2}\left(\Omega ; \mathbb{R}^{N}\right)\right)$ be a weak solution of the non-linear parabolic system $(1.1)$ in $\Omega_{T}$ under the assumptions (1.2)-(1.4). Then, there holds

$$
\partial_{\mathcal{P}} \Omega_{T} \backslash \operatorname{Reg}_{\mathcal{P}} u \subset \Sigma^{1} \cup \Sigma^{2},
$$

where 


$$
\Sigma^{1}=\left\{z_{0} \in \partial_{\mathcal{P}} \Omega_{T}: \liminf _{\varrho \downarrow 0} f_{\Omega_{T} \cap Q_{\varrho}\left(z_{0}\right)}\left|D(u-g)-(D(u-g))_{\Omega_{T} \cap Q_{\varrho}\left(z_{0}\right)}\right|^{2} d z>0\right\}
$$

and

$$
\Sigma^{2}=\left\{z_{0} \in \partial_{\mathcal{P}} \Omega_{T}: \limsup _{\varrho \downarrow 0}\left|(D(u-g))_{\Omega_{T} \cap Q_{\varrho}\left(z_{0}\right)}\right|=\infty\right\} .
$$

Furthermore, if $z_{0} \in \operatorname{Reg}_{\mathcal{P}} u$ then $D u \in C^{\beta, \frac{\beta}{2}}\left(\overline{U \cap \Omega_{T}} ; \mathbb{R}^{N n}\right)$ for some neighborhood $U$ of $z_{0}$.

For related interior parabolic and elliptic results - obtained by a similar method - we refer to $[4,16-19,13,30]$. The previous result is the starting point for the almost everywhere boundary regularity result described at the beginning of this Introduction. Indeed, to prove that a boundary point $z_{0} \in \partial_{\mathcal{P}} \Omega_{T}$ is regular it suffices to prove that the following conditions hold:

$$
\liminf _{\varrho \downarrow 0} f_{\Omega_{T} \cap Q_{\varrho}\left(z_{0}\right)}\left|D(u-g)-(D(u-g))_{\Omega_{T} \cap Q_{\varrho}\left(z_{0}\right)}\right|^{2} d z=0
$$

and

$$
\underset{\varrho \downarrow 0}{\limsup }\left|(D(u-g))_{\Omega_{T} \cap Q_{\varrho}\left(z_{0}\right)}\right|<\infty .
$$

The strategy of the paper consists of proving an up to the boundary fractional differentiability result for $D u$ which in turn implies that conditions (1.5) and (1.6) are satisfied at almost every point $z_{0} \in \partial_{\mathcal{P}} \Omega_{T}$, where "almost everywhere" refers to the standard boundary surface measure on $\partial_{\mathcal{P}} \Omega_{T}$. We remark that a similar strategy was developed for the elliptic case to get first singular sets estimates in the interior [28,29], and then at the boundary [15], and [16,18] for the interior parabolic case; we refer to [25-27] for results concerning the stationary variational case. As usual, when dealing with parabolic initial boundary value problems we shall distinguish between the lateral boundary situation, i.e. points lying on the lateral boundary $\partial_{\text {lat }} \Omega_{T}=\partial \Omega \times(0, T)$ and the initial boundary situation including such points lying near the initial boundary $\Omega_{0}=\Omega \times\{0\}$. The natural quantity to measure the size of the singular sets

$$
\operatorname{Sing}_{\text {lat }} u=\partial_{\text {lat }} \Omega_{T} \backslash \operatorname{Reg}_{\mathcal{P}} u \text { and } \quad \operatorname{Sing}_{\text {ini }} u=\Omega_{0} \backslash \operatorname{Reg}_{\mathcal{P}} u
$$

is the parabolic Hausdorff-dimension, i.e. the Hausdorff-dimension $\operatorname{dim}_{\mathcal{P}}$ related to the parabolic Hausdorff-measures which are constructed with respect to the parabolic metric by the usual construction of Carathéodory (see (2.1) below for the definition). Taking into account that $\operatorname{dim}_{\mathcal{P}}\left(\partial_{\text {lat }} \Omega_{T}\right)=n+1$, respectively $\operatorname{dim}_{\mathcal{P}}\left(\Omega_{0}\right)=n$ we are looking for conditions ensuring a bound of the form

$$
\operatorname{dim}_{\mathcal{P}}\left(\operatorname{Sing}_{\text {lat }} u\right)<n+1, \quad \text { respectively } \operatorname{dim}_{\mathcal{P}}\left(\operatorname{Sing}_{\text {ini }} u\right)<n .
$$

Note that we do not need to take into account edge points, i.e. those lying on $\partial \Omega \times\{0\}$ since we already have $\operatorname{dim}_{\mathcal{P}}(\partial \Omega \times\{0\})=n-1$.

Our first main result is concerned with the existence of regular lateral boundary points and is

Theorem 1.3 (Lateral boundary existence). Let $u \in L^{2}\left(0, T ; W^{1,2}\left(\Omega ; \mathbb{R}^{N}\right)\right)$ be a weak solution of the non-linear parabolic system (1.1) in $\Omega_{T}$ under the assumptions (1.2), (1.3), (1.4) $)_{1}$ and (1.4) $)_{3}$. Moreover, assume that Dg $\in$ $C^{\beta, \frac{\beta}{2}}\left(\Omega_{T} ; \mathbb{R}^{N n}\right)$ and $g_{t} \in \mathcal{N}^{0, \xi ; 2}\left(\Omega_{T} ; \mathbb{R}^{N}\right)$ for some $\xi \in(0,1)$. If

$$
\beta>\frac{1}{2}
$$

then $\mathcal{H}_{\mathcal{P}}^{n+1}$-almost every lateral boundary point is a regular point of Du. Moreover, if additionally $g_{t} \in$ $\mathcal{N}^{0, \xi ; \sigma}\left(\Omega_{T} ; \mathbb{R}^{N}\right)$ for some $\xi \in(0,1)$ and $\sigma>2$, then there exists $\delta=\delta\left(n, L / v,\|g\|_{C^{1 ; \beta, \beta / 2}},\left\|g_{t}\right\|_{\mathcal{N}^{0, \xi ; \sigma}}, \partial \Omega\right)>0$ such that if

$$
\beta>\frac{1}{2}-\delta
$$

then $\mathcal{H}_{\mathcal{P}}^{n+1}$-almost every lateral boundary point is a regular point of $\mathrm{Du}$. 
For the definition of the Nikolskii space $\mathcal{N}^{0, \xi ; \sigma}\left(\Omega_{T} ; \mathbb{R}^{N n}\right)$ we refer to Definition 2.1. We emphasize here that the crucial point is not the a priori regularity assumed on the boundary datum $g$ - that for simplicity could be assumed smooth as well - but rather the almost everywhere regularity at the boundary; anyway we here tried to minimize the assumptions on $g$. The reinforcement of our assumption on the boundary data, in the sense that $D g$ is also Hölder continuous with respect to time, is needed in order to transform the Hölder continuity assumption (1.2) 4 with respect to time to a model situation with homogeneous boundary data (see Section 2.1). The $\delta$-improvement in (1.7) is achieved by the use of an up to the boundary version of Gehring's lemma. Since the dependencies of the up to the boundary higher integrability on the structure parameters can be given explicitly $[9,33]$, we have that $\delta \rightarrow 0$ when $L / v \rightarrow \infty$. The same applies with respect to the dependency of the constants on $\|g\|_{C^{1 ; \beta, \beta / 2}},\left\|g_{t}\right\|_{\mathcal{N}^{0, \xi ; \sigma}}$ or $\partial \Omega$; this means that $\delta \rightarrow 0$ when $g$, respectively $\partial \Omega$ degenerate in the $C^{1 ; \beta, \beta / 2}$ - respectively $C^{1, \beta}$-sense (see Section 2.1 ).

Remark 1.4. It is worth mentioning that the Morrey-condition imposed on the time derivative $g_{t}$ of $g$ in Theorem 1.3 is only needed to ensure that the characterization of regular points at the lateral boundary from Theorem 1.2 is in force. For the estimate of the singular set, i.e. the dimension reduction argument, it would be sufficient to assume that $g_{t} \in \mathcal{N}^{0, \xi ; 2}\left(\Omega_{T} ; \mathbb{R}^{N}\right)$, respectively $g_{t} \in \mathcal{N}^{0, \xi ; \sigma}\left(\Omega_{T} ; \mathbb{R}^{N}\right)$.

The proof of Theorem 1.3 is based on several steps, culminating in an up to the lateral boundary fractional differentiability estimate for the spatial gradient $D u$. In order to prove such a fractional differentiability we use an analog of the comparison technique introduced for the treatment of the boundary regularity problem in the time independent elliptic setting from [15], but, we have to remark, the adaptation of such a technique to the parabolic case is far from being straightforward, and involves highly non-trivial additional difficulties. A crucial idea in the argument is that space-time estimates for finite differences of $D u$ are turned, via a delicate comparison argument, into the same kind of estimates for more regular solutions $u_{h}$ of an associated regularized problem

$$
\partial_{t} u_{h}-\operatorname{div} a_{h}\left(x, t, D u_{h}\right)=\partial_{t} g(x, t) \quad \text { in } Q .
$$

Here the vector field $a_{h}$ is Lipschitz continuous with respect to the coefficients $(x, t)$, and $h>0$ is a parameter to be fixed and to which the size of the Lipschitz seminorm of the partial map $(x, t) \mapsto a_{h}(x, t, \cdot)$ is linked; in other words, the regularity of $a_{h}$ degenerates when $h$ converges to zero. The derivation of these estimates, i.e. the higher differentiability for $D u_{h}$, is definitely not straightforward. These results, especially in the peculiar form needed here, are not present in the literature, and their proof is rather delicate: when turning to the boundary estimates for the parabolic case one has to retrieve regularity information on "two missing directions", namely the normal - with respect to the boundary of the base space domain $\partial \Omega$ - and the time direction. The strategy is, in the rough description we are giving in the following lines, to treat first the tangential directions - here tangential means with respect to the tangential directions to $\partial \Omega$ - by standard difference quotient techniques. Unfortunately, these are insufficient for the use of the system itself in order to obtain estimates for the normal component of the derivative of the weak solution, as for instance happens in the elliptic case - see for instance [26, Section 4.3] or [15] - since now there is an additional missing direction - the time one - on whose behavior nothing is a priori known. This delicate step will be achieved exploiting certain reiteration/semigroup properties of finite difference operators, and this will lead to a delicate interplay between space and time difference quotients using both intrinsic properties of fractional Sobolev spaces and tools from Harmonic Analysis as a properly localized version of the Fefferman-Stein theorem on sharp maximal operators. The final outcome is the existence of the weak time-derivative of $u_{h}$ in $L^{2}$. Subsequently, we are in the position to exploit the parabolic system in order to obtain suitable estimates for the normal derivatives. The technical details can be found in Section 4. The main results are the up to the lateral boundary existence of second-order spatial derivatives $D^{2} u_{h}$ and of the time-derivative $\partial_{t} u_{h}$ in $L^{2}$ in Theorem 4.2 - and furthermore in $L^{\sigma}$ for some $\sigma>2$ in Proposition 4.15. After this preliminary step we can start the real regularization of our original solution $u$ to (1.1). The idea is to make a comparison between $D u$ and the gradient $D u_{h}$ of the regularized problems, where the parameter $h$ is chosen accordingly to the rate of the finite difference considered $D u(x+h, t)-D u(x, t)$ and to make such a finite difference inherit the decay properties of $D u_{h}(x+h, t)-D u_{h}(x, t)$. At this stage we have to exploit a delicate balance between the decay properties of $D u_{h}(x+h, t)-D u_{h}(x, t)$ due to $D^{2} u_{h} \in L^{2}$ and the fact that in general $\left\|D^{2} u_{h}\right\|_{L^{2}} \rightarrow \infty$ as $h \rightarrow \infty$; the final result is the fractional differentiability of the spatial gradient of the original solution $D u$. A similar argument is then applied to get the fractional time differentiability. 
Our second main result concerns the existence of regular initial boundary points. As mentioned before, the initial boundary $\Omega_{0}$ only has parabolic Hausdorff dimension equal to $n$. Therefore, contrary to the lateral boundary situation we now have to reduce the dimension of the singular set below $n$. This yields a positive result only when $\beta$ is close to 1 . To be precise, at the initial boundary we can show the following:

Theorem 1.5 (Initial time existence). Let $u \in L^{2}\left(0, T ; W^{1,2}\left(\Omega ; \mathbb{R}^{N}\right)\right)$ be a weak solution of the non-linear parabolic system (1.1) in $\Omega_{T}$ under the assumptions $(1.2)_{1}-(1.2)_{3},(1.3)$ and $(1.4)_{2}$. Then there exists $\delta=$ $\delta\left(n, L / v,\|g\|_{C^{1 ; \beta, 0}}\right)>0$ such that if

$$
\beta>1-\delta
$$

then $\mathcal{H}_{\mathcal{P}}^{n}$-almost every initial boundary point is a regular point of $\mathrm{Du}$.

The proof for the initial boundary situation is in a certain sense easier than the one for the lateral boundary. As we did there we again compare the solution $u$ to solutions $u_{h}$ of a regularized problem of the type (1.8). But now we can apply the difference quotient method with respect to all space directions in order to infer the existence of the second space derivative $D^{2} u_{h}$ in $L^{2}$. Then, the existence of the time derivative $\partial_{t} u_{h}$ in $L^{2}$ easily follows from the parabolic system. At this stage it is worth mentioning that this procedure does not involve a difference quotient method with respect to time. For this reason hypothesis $(1.2)_{4}$ is not needed in Theorem 1.5.

\section{Notation and preliminary material}

In this paper we will follow the definitions and the notation established in the first part [7]; we shall only repeat here the very basic notation concerning balls and cylinders. In general we shall write $x=\left(x_{1}, \ldots, x_{n}\right)$ for a point in $\mathbb{R}^{n}$ and $z=(x, t)=\left(x_{1}, \ldots, x_{n}, t\right)$ for a point in $\mathbb{R}^{n+1}$. By $B_{\varrho}\left(x_{0}\right) \equiv\left\{x \in \mathbb{R}^{n}:\left|x-x_{0}\right|<\varrho\right\}$, respectively $B_{\varrho}^{+}\left(x_{0}\right) \equiv$ $B_{\varrho}\left(x_{0}\right) \cap\left\{x \in \mathbb{R}^{n}: x_{n}>0\right\}$ we denote the open ball, respectively half-ball in $\mathbb{R}^{n}$ with center $x_{0} \in \mathbb{R}^{n}$ and radius $\varrho>0$. When considering $B_{\varrho}^{+}\left(x_{0}\right)$, unless otherwise specified, we shall always have $x_{0}$ with $\left(x_{0}\right)_{n}=0$. Moreover, we write $\Lambda_{\varrho^{2}}\left(t_{0}\right)=\left(t_{0}-\varrho^{2}, t_{0}+\varrho^{2}\right)$ for the open interval around $t_{0} \in \mathbb{R}$ of length $2 \varrho^{2}$ and $\Lambda_{\varrho^{2}}^{0}\left(t_{0}\right)=\Lambda_{\varrho^{2}}\left(t_{0}\right) \cap\{t \in \mathbb{R}: t>0\}$. As before, we always have $t_{0}=0$ when writing $\Lambda_{\varrho^{2}}^{0}\left(t_{0}\right)$, unless otherwise stated. The (half-)cylinders are denoted by $Q_{\varrho}\left(z_{0}\right) \equiv B_{\varrho}\left(x_{0}\right) \times \Lambda_{\varrho^{2}}\left(t_{0}\right)$ and $Q_{\varrho}^{+}\left(z_{0}\right) \equiv B_{\varrho}^{+}\left(x_{0}\right) \times \Lambda_{\varrho^{2}}\left(t_{0}\right)$ and $Q_{\varrho}^{0}\left(z_{0}\right) \equiv B_{\varrho}\left(x_{0}\right) \times \Lambda_{\varrho^{2}}^{0}\left(t_{0}\right)$, where $z_{0}=$ $\left(x_{0}, t_{0}\right) \in \mathbb{R}^{n+1}, \varrho>0$. Moreover, we write $\Gamma_{\varrho}\left(z_{0}\right) \equiv Q_{\varrho}\left(z_{0}\right) \cap\left\{\left(x_{1}, \ldots, x_{n}, t\right) \in \mathbb{R}^{n+1}: x_{n}=0\right\}$ for the lateral part of the boundary of $Q_{\varrho}^{+}\left(z_{0}\right)$ and for the initial boundary of $Q_{\varrho}^{0}\left(z_{0}\right)$ we write $D_{\varrho}\left(z_{0}\right)=Q_{\varrho}\left(z_{0}\right) \cap\left\{(x, t) \in \mathbb{R}^{n+1}: t=0\right\}$. If $z_{0}=0$, a typical situation occurring when treating the regularity of lateral boundary points after "flattening the boundary", we abbreviate $B_{\varrho}=B_{\varrho}(0), \Lambda_{\varrho^{2}}=\Lambda_{\varrho^{2}}(0), Q_{\varrho}=Q_{\varrho}(0), \Gamma_{\varrho}=\Gamma_{\varrho}(0)$ and $D_{\varrho}=D_{\varrho}(0)$.

For an integrable map $v: A \rightarrow \mathbb{R}^{k}, k \in \mathbb{N}$, we write

$$
(v)_{A} \equiv \int_{A} v d z=\frac{1}{|A|} \int_{A} v d z
$$

for its mean value on $A$, provided $|A|>0$. If $A=Q_{\varrho}\left(z_{0}\right)$ then we write $(v)_{z_{0}, \varrho}$ for the mean value of $v$ on the parabolic cylinder $Q_{\varrho}\left(z_{0}\right)$ and $(v)_{z_{0}, \varrho}^{+}$for the mean value on the parabolic half-cylinder $Q_{\varrho}^{+}\left(z_{0}\right)$ and $(v)_{z_{0}, \varrho}^{0}$ for the mean value on $Q_{\varrho}^{0}\left(z_{0}\right)$. Finally, we write $\partial_{\text {lat }} \Omega_{T}=\partial \Omega \times(0, T)$ for the lateral boundary of $\Omega_{T}$ and $\Omega_{0}=\Omega \times\{0\}$ for its initial boundary.

Definition 2.1. With $q \geqslant 1, \vartheta \in(0,1)$ and $Q=\Omega \times\left(t_{1}, t_{2}\right) \subset \mathbb{R}^{n+1}$ being a parabolic cylinder, a measurable map $v: Q \rightarrow \mathbb{R}^{k}, k \geqslant 1$, belongs to the (parabolic) Nikolskii space $\mathcal{N}^{0, \vartheta ; q}\left(Q ; \mathbb{R}^{k}\right)$ if and only if with $Q_{\eta}=$ $\Omega \times\left(t_{1}+\eta, t_{2}-\eta\right)$ it holds that

$$
\|v\|_{\mathcal{N}^{0, \vartheta ; q}\left(Q ; \mathbb{R}^{k}\right)}^{q}:=\|v\|_{L^{q}\left(Q ; \mathbb{R}^{k}\right)}^{q}+\sup _{\eta>0,0<|h| \leqslant \eta} \int_{Q_{\eta}} \frac{|v(x, t+h)-v(x, t)|^{q}}{|h|^{\vartheta q}} d(x, t)<\infty .
$$


Finally, the parabolic Hausdorff-dimension related to the parabolic metric is defined by

$$
\operatorname{dim}_{\mathcal{P}}(F) \equiv \inf \left\{s>0: \mathcal{H}_{\mathcal{P}}^{s}(F)=0\right\}=\sup \left\{s>0: \mathcal{H}_{\mathcal{P}}^{s}(F)=\infty\right\},
$$

where $F \subset \mathbb{R}^{n+1}$ and

$$
\mathcal{H}_{\mathcal{P}}^{s}(F) \equiv \liminf _{\varrho \downarrow 0}\left\{\sum_{i=1}^{\infty} \varrho_{i}^{s}: F \subset \bigcup_{i=1}^{\infty} Q_{\varrho_{i}}\left(z_{i}\right), 0 \leqslant \varrho_{i}<\varrho\right\}
$$

denotes the parabolic $s$-dimensional Hausdorff-measure, $s \in[0, n+2]$, of $F$.

\subsection{Transformation to the model situation}

Since our results are of local nature we are allowed to consider the lateral and the initial boundary situation separately, i.e. to prove regularity for a point $z_{0}=\left(x_{0}, 0\right) \in \Omega_{0}$ lying on the initial boundary then it is enough to consider parabolic cylinders $Q_{\varrho}^{0}\left(z_{0}\right)$ with $B_{\varrho}\left(x_{0}\right) \Subset \Omega$ and the same for points lying on the lateral boundary. When considering the lateral boundary we will prove our results in a model situation on the half-cylinder $Q_{1}^{+}$and for boundary values $u \equiv 0$ on the lateral boundary $\Gamma_{1}$. Therefore, we will always refer to a Cauchy-Dirichlet problem of the following type:

$$
\begin{cases}u_{t}-\operatorname{div} a(z, u, D u)=g_{t} & \text { in } Q_{1}^{+}, \\ u=0 & \text { on } \Gamma_{1},\end{cases}
$$

where $\partial_{t} g \in L^{2,2-2 \beta}\left(Q_{1}^{+} ; \mathbb{R}^{n}\right)$. For the precise transformation leading to this model situation we refer to [7, Section 2.1]. In the initial boundary situation the proceeding is simpler. Here, we shall transform the problem to the model situation where the initial values are equal to zero, i.e. we consider

$$
\begin{cases}u_{t}-\operatorname{div} a(z, u, D u)=0 & \text { in } \Omega_{T}, \\ u(\cdot, 0)=0 & \text { on } \Omega\end{cases}
$$

which is achieved by a transformation $v(x, t)=u(x, t)-g(x, 0)$. Note that this is not exactly the same model situation that we had chosen in [7]. Indeed, there we considered the transformation $v(x, t)=u(x, t)-g(x, t)$ leading to a nonhomogeneous model problem; the reason was that we wanted to have the same model problem in every possible case, in order to join them at the edge: as a matter of fact Theorem 1.2 work for the edge points too; in the present situation we do not need to consider edge points since the edge has already properly low parabolic Hausdorff dimension. As mentioned in [7, Section 2.1], the final characterization of the singular set is the same for both transformations.

Let us now comment on the regularity assumed for $D g$. In the setting of Theorem 1.3 - i.e. when dealing with the lateral boundary - where the vector-field $a$ is assumed to be Hölder continuous with respect to $x$ and $t$ we need to assume a certain continuity of $D g$ with respect to $t$, i.e. $D g \in C^{\beta, \frac{\beta}{2}}\left(\Omega_{T} ; \mathbb{R}^{N n}\right)$ in order to have the transformed vector field to be Hölder continuous with respect to $x$ and $t$. On the other hand, in the setting of Theorem $1.5-$ i.e. when dealing with the initial boundary - we can renounce on the Hölder continuity assumption (1.2) 4 of the vector field $a$ with respect to time. Therefore, also the Hölder continuity of $D g$ with respect to time is not needed such that the weaker assumption $D g \in C^{\beta, 0}\left(\Omega_{T} ; \mathbb{R}^{N n}\right)$ is enough.

Finally, we want to comment on the change of the structure constants when passing to the model situation. The new growth constant $\tilde{L}$ then is of the form $L \cdot c\left(p,\|g\|_{C^{1 ; \beta, \beta / 2}}, \partial \Omega\right)$ in Theorem 1.3, respectively $L \cdot c\left(p,\|g\|_{C^{1 ; \beta}}, \partial \Omega\right)$ in Theorem 1.5, while the new ellipticity constant $\tilde{v}$ is of the form $L / c\left(p,\|g\|_{C^{1 ; \beta, \beta / 2}}, \partial \Omega\right)$ in Theorem 1.3 , respectively $L \cdot c\left(p,\|g\|_{C^{1 ; \beta}}, \partial \Omega\right)$ in Theorem 1.5 , where the constant $c(\cdots)$ is strictly larger then 0 . Therefore, in the estimates for the original problem (1.1) the constants will depend on $L / v \cdot c\left(p,\|g\|_{C^{1 ; \beta, \beta / 2}}, \partial \Omega\right)^{2}$, respectively $L / v \cdot c\left(p,\|g\|_{C^{1 ; \beta}}, \partial \Omega\right)^{2}$.

\subsection{Steklov averages}

Let us recall from [7] the definition of the so-called Steklov-means. Given a function $f \in L^{1}\left(\Omega \times\left(t_{1}, t_{2}\right)\right)$ and $0<|h| \leqslant \frac{1}{2}\left(t_{2}-t_{1}\right)$, we define its Steklov-mean by 


$$
[f]_{h}(x, t) \equiv \begin{cases}\frac{1}{|h|} \int_{t}^{t+h} f(x, s) d s, & t \in\left[t_{1}+|h|, t_{2}-|h|\right], \\ 0, & t \in\left(t_{1}, t_{1}+|h|\right) \cup\left(t_{2}-|h|, t_{2}\right) .\end{cases}
$$

The previous definition should be used when dealing with symmetric parabolic cylinders which are far from the initial boundary. When dealing with the initial boundary problem we shall adopt the following one, valid in the case $0<h \leqslant t_{2}-t_{1}$ :

$$
[f]_{h}(x, t) \equiv \begin{cases}\frac{1}{h} \int_{t}^{t+h} f(x, s) d s, & t \in\left(t_{1}, t_{2}-h\right], \\ 0, & t \in\left(t_{2}-h, t_{2}\right) .\end{cases}
$$

\subsection{Preliminary lemmas}

Contrary to the interior parabolic case, in the lateral boundary situation we have an automatic Poincare inequality for those functions

$$
u \in L^{p}\left(\Lambda_{\varrho^{2}}\left(t_{0}\right) ; W^{1, p}\left(B_{\varrho}\left(x_{0}\right)^{+} ; \mathbb{R}^{k}\right)\right)
$$

satisfying $u \equiv 0$ on the lateral boundary $\Gamma_{\varrho}\left(z_{0}\right)$. This inequality can be obtained applying the standard Poincaré inequality to the functions $u(\cdot, t) \in W^{1, p}\left(B_{\varrho}^{+}\left(x_{0}\right) ; \mathbb{R}^{k}\right)$ for a.e. $t \in \Lambda_{\varrho^{2}}\left(t_{0}\right)$ and then integrating with respect to $t$.

Lemma 2.2. Let $z_{0}=\left(x_{0}, t_{0}\right) \in \mathbb{R}^{n+1}$ with $x_{0} \in \mathbb{R}^{n-1} \times\{0\}$. Then for any function $u \in L^{p}\left(\Lambda_{\varrho^{2}}\left(t_{0}\right)\right.$; $\left.W^{1, p}\left(B_{\varrho}\left(x_{0}\right)^{+} ; \mathbb{R}^{k}\right)\right), k \geqslant 1$, satisfying $u \equiv 0$ on $\Gamma_{\varrho}\left(z_{0}\right)$ there holds

$$
f_{Q_{\varrho}^{+}\left(z_{0}\right)}|u|^{p} d z \leqslant \frac{\varrho^{p}}{p} f_{Q_{\varrho}^{+}\left(z_{0}\right)}\left|D_{n} u\right|^{p} d z
$$

The next lemma is a boundary version of the Sobolev-embedding theorem.

Lemma 2.3. Let $v \in W^{1, \frac{2 n}{n+2}}\left(B_{\varrho}^{+}, \mathbb{R}^{k}\right)$ with $0<\varrho \leqslant 1, k \in \mathbb{N}$, satisfying $v=0$ on $\partial B_{\varrho} \cap\left\{x \in \mathbb{R}^{n}: x_{n}>0\right\}$. Then $v \in L^{2}\left(B_{\varrho}^{+}, \mathbb{R}^{k}\right)$ and there holds

$$
f_{B_{\varrho}^{+}}|v|^{2} d x \leqslant c(n) \varrho^{2}\left(f_{B_{\varrho}^{+}}|D v|^{\frac{2 n}{n+2}} d x\right)^{\frac{n+2}{n}} .
$$

Proof. First, we extend $v$ from $B_{\varrho}^{+}$to $B_{\varrho}$ by an even reflection, i.e. we define

$$
\tilde{v}\left(x^{\prime}, x_{n}\right) \equiv \begin{cases}v\left(x^{\prime}, x_{n}\right) & \text { if } x_{n} \geqslant 0 \\ v\left(x^{\prime},-x_{n}\right) & \text { if } x_{n}<0\end{cases}
$$

Then, from the reflection principle we know that $\tilde{v} \in W^{1, \frac{n}{n+2}}\left(B_{\varrho} ; \mathbb{R}^{k}\right)$. Therefore we can apply the Sobolev-Poincaré inequality to $\tilde{v}$ on $B_{\varrho}$ to infer that

$$
\int_{B_{\varrho}}|\tilde{v}| d x \leqslant c(n)\left(\int_{B_{\varrho}^{+}}|D \tilde{v}|^{\frac{n}{n+2}} d x\right)^{\frac{n+2}{n}} .
$$

Since $\tilde{v}\left(x^{\prime}, x_{n}\right)=v\left(x^{\prime},-x_{n}\right)$ on $B_{\varrho} \backslash B_{\varrho}^{+}$, this estimate yields the desired boundary version of the Sobolev-Poincaré inequality.

Next, we recall some basic facts about finite differences. Let $f: \mathbb{R}^{n+1} \supset \Omega \times\left(t_{1}, t_{2}\right) \rightarrow \mathbb{R}^{k}, k \in \mathbb{N}$. We define the finite differences in space direction $\tau_{h}^{\alpha}(f)$ by

$$
\left(\tau_{h}^{\alpha} f\right)(x, t) \equiv f\left(x+h e_{\alpha}, t\right)-f(x, t),
$$


whenever $x \in \Omega$ and $x+h e_{\alpha} \in \Omega$ and $t \in\left(t_{1}, t_{2}\right)$, where $|h|>0,1 \leqslant \alpha \leqslant n$, and $\left\{e_{\alpha}\right\}_{1 \leqslant \alpha \leqslant n}$ is the standard basis of $\mathbb{R}^{n}$. Similarly the finite difference in time direction $\tau_{h}(f)$ is defined by

$$
\left(\tau_{h} f\right)(x, t) \equiv f(x, t+h)-f(x, t),
$$

whenever $x \in \Omega, t, t+h \in\left(t_{1}, t_{2}\right)$ and $|h|>0$. For finite differences in space direction we have the following standard estimate, which is in turn a consequence of basic facts from the theory of Sobolev functions: Let $f, D_{\alpha} f \in L^{p}\left(B_{R+|h|}^{+}\left(x_{0}\right) \times\left(t_{1}, t_{2}\right)\right)$ where $\alpha \in\{1, \ldots, n\},|h|>0$, and $h>0$ when dealing with the case $\alpha=n$. Then we have

$$
\int_{t_{1}}^{t_{2}} \int_{B_{R}^{+}\left(x_{0}\right)}\left|\tau_{h}^{\alpha} f\right|^{p} d x d t \leqslant|h|^{p} \int_{t_{1}}^{t_{2}} \int_{B_{R+|h|}^{+}\left(x_{0}\right)}\left|D_{\alpha} f\right|^{p} d x d t
$$

Moreover, we can bound second differences in terms of first differences as follows

$$
\left|\tau_{-h} \tau_{h} f(t)\right|=|2 f(t)-f(t+h)-f(t-h)| \leqslant\left|\tau_{h} f(t)\right|+\left|\tau_{-h} f(t)\right| .
$$

The following lemma can be obtained by a slight modification of the proof of [12, Theorem 1.1], and its proof can be obtained from the proof of this last result. The result basically deals with a semigroup property of finite difference operators, see also [34].

Lemma 2.4. Let $B$ be a Banach space, $T>0,0<h_{0}<T / 2, f \in L^{\sigma}\left(-T, T+2 h_{0} ; B\right), \sigma \geqslant 1$ and $\alpha>0$. Suppose that there exists $M>0$ such that

$$
\left\|\tau_{h}\left(\tau_{h} f\right)\right\|_{L^{\sigma}(-T, T ; B)} \leqslant M|h|^{\alpha} \quad \text { whenever } 0<h \leqslant h_{0} .
$$

Then

$$
\left\|\tau_{h} f\right\|_{L^{\sigma}(-T, T ; B)} \leqslant c\left(h_{0}^{\alpha-\beta} M+h_{0}^{-\beta}\|f\|_{L^{\sigma}\left(-T, T+2 h_{0} ; B\right)}\right)|h|^{\beta}
$$

holds whenever $0<h \leqslant h_{0} / 4$, with $c=c(\alpha, \beta)$ and $\beta=\min \{1, \alpha\}$ for $\alpha \neq 1$, and for any $\beta \in(0,1)$ for $\alpha=1$. The same holds by taking $h$ non-positive i.e. assuming $0<-h \leqslant h_{0}$, and replacing norms in $L^{\sigma}\left(-T, T+2 h_{0} ; B\right)$ with norms in $L^{\sigma}\left(-T-2 h_{0}, T ; B\right)$. Finally, the same result holds for functions defined in $L^{\sigma}(0, T ; B)$, replacing everywhere $-T$ by 0 .

\subsection{Fractional Sobolev spaces}

The proof of our dimension reduction result is based on the idea to establish additional fractional differentiability properties of the spatial derivative $D u$ of our weak solution $u$ and then to exploit this by using a certain fractional Poincaré inequality in order to show that the criterion ensuring regularity is fulfilled on a large set. For convenience of the reader we recall the definition of fractional Sobolev spaces which are suited for the treatment of parabolic problems. Let $1 \leqslant p<\infty, k \in \mathbb{N}$, and $\alpha, \gamma \in(0,1)$. Then, we say that a function $v \in L^{p}\left(\Omega_{T} ; \mathbb{R}^{k}\right)$ belongs to the parabolic fractional Sobolev space $W^{\alpha, \gamma ; p}\left(\Omega_{T} ; \mathbb{R}^{k}\right)$, if

$$
[v]_{\alpha, \gamma ; p ; \Omega_{T}}^{p}:=\int_{0}^{T} \int_{\Omega} \int_{\Omega} \frac{|v(x, t)-v(y, t)|^{p}}{|x-y|^{n+\alpha p}} d x d y d t+\int_{\Omega} \int_{0}^{T} \int_{0}^{T} \frac{|v(x, t)-v(x, \tau)|^{p}}{|t-\tau|^{1+\gamma p}} d t d \tau d x<\infty .
$$

The local variant, i.e. the space $W_{\mathrm{loc}}^{\alpha, \gamma ; p}\left(\Omega_{T} ; \mathbb{R}^{k}\right)$ is defined as usual. This means that $v \in W_{\mathrm{loc}}^{\alpha, \gamma ; p}\left(\Omega_{T} ; \mathbb{R}^{k}\right)$, if $v \in$ $W^{\alpha, \gamma ; p}\left(\tilde{Q} ; \mathbb{R}^{k}\right)$ for all sub-cylinders $\tilde{Q} \Subset \Omega_{T}$.

We will need the following parabolic boundary version of the well-known relation between fractional Sobolev spaces and Nikolskii spaces. The proof can be adapted from the standard proof in the interior case, presented for instance in [25, Lemma 2.5]. 
Lemma 2.5. Let $v \in L^{p}\left(Q_{2 R}^{+} ; \mathbb{R}^{k}\right), 1 \leqslant p<\infty, k \in \mathbb{N}, R>0$. Then the following assertions hold:

(i) Suppose that

$$
\int_{B_{R}^{+}} \int_{-R^{2}}^{R^{2}}\left|\tau_{h} v\right|^{p} d t d x \leqslant c_{1}\|v\|_{L^{p}\left(Q_{2 R}^{+}\right)}^{p}|h|^{p \gamma}, \quad \gamma \in(0,1),
$$

for every $h \in \mathbb{R}$ such that $0<|h| \leqslant \min \left\{R^{2}, A_{1}\right\}$ where $A_{1}, c_{1}>0$ are positive constants. Then for every $\tilde{\gamma} \in$ $(0, \gamma)$ there exists $\tilde{c}_{1}=\tilde{c}_{1}\left(n, p, \gamma, \tilde{\gamma}, A_{1}, c_{1}, R^{2}\right)$, such that

$$
\iint_{B_{R}^{+}} \int_{-R^{2}}^{R^{2}} \int_{-R^{2}}^{R^{2}} \frac{|v(x, t)-v(x, \tau)|^{p}}{|t-\tau|^{1+\tilde{\gamma} p}} d \tau d t d x \leqslant \tilde{c}_{1} .
$$

(ii) Suppose that

$$
\int_{-R^{2}}^{R^{2}} \int_{B_{R}^{+}}\left|\tau_{h}^{\alpha} v\right|^{p} d x d t \leqslant c_{2}\|v\|_{L^{p}\left(Q_{2 R}^{+}\right)}^{p}|h|^{p \gamma} \quad \text { for some } \gamma \in(0,1),
$$

whenever $h \in \mathbb{R}$ such that $0<|h| \leqslant \frac{R}{A_{2}}$ and $\alpha \in\{1, \ldots, n\}$ where $A_{2} \geqslant 1$ and $c_{2}>0$. In the case $\alpha=n$ we impose the preceding estimate for $h>0$ only. Then, for any $\tilde{\gamma} \in(0, \gamma)$ there exists $\tilde{c}_{2}=\tilde{c}_{2}\left(n, p, R, \gamma, \tilde{\gamma}, A_{2}, c_{2}\right)$, such that

$$
\int_{-R^{2}}^{R^{2}} \int_{B_{R / 2}^{+}} \int_{B_{R / 2}^{+}} \frac{|v(x, t)-v(y, t)|^{p}}{|x-y|^{n+\tilde{\gamma} p}} d x d y d t \leqslant \tilde{c}_{2} .
$$

To conclude estimates for the Hausdorff-dimension of the singular set we will use the boundary version of [16, Proposition 3.3].

Lemma 2.6. Let $u \in W^{\beta, \beta / 2 ; 2}\left(Q_{R}^{+} ; \mathbb{R}^{k}\right)$ with $\beta \in(0,1)$ and let

$$
\begin{aligned}
& A:=\left\{z_{0} \in \Gamma_{R}: \liminf _{\varrho \downarrow 0} \underset{Q_{\varrho}^{+}\left(z_{0}\right)}{f}\left|u-(u)_{z_{0}, \varrho}\right|^{2} d z>0\right\}, \\
& B:=\left\{z_{0} \in \Gamma_{R}: \limsup _{\varrho \downarrow 0}\left|(u)_{z_{0}, \varrho}^{+}\right|=\infty\right\} .
\end{aligned}
$$

Then

$$
\operatorname{dim}_{\mathcal{P}}(A) \leqslant n+2-2 \beta \text { and } \operatorname{dim}_{\mathcal{P}}(B) \leqslant n+2-2 \beta
$$

\section{Higher integrability up to the boundary}

In this section we are concerned with the higher integrability properties up to the lateral boundary of weak solutions to certain non-linear parabolic systems with $p$-growth. Such a higher integrability is needed in order to obtain the $\delta$ improvement in (1.7) in Theorem 1.3 when establishing an improvement of the estimate for the dimension of the singular set at the lateral boundary. The main outcome with this respect is the following up to the lateral boundary higher integrability result. For related higher integrability results see [1,31]. 
Lemma 3.1. Suppose that $f \in L^{\sigma_{1}}\left(Q_{R}^{+} ; \mathbb{R}^{N}\right)$ and $b \in L^{\sigma_{1}}\left(Q_{R}^{+} ; \mathbb{R}^{N n}\right)$ for some $\sigma_{1}>2, R>0$ and that $v \in$ $L^{2}\left(\Lambda_{R^{2}} ; W^{1,2}\left(B_{R}^{+} ; \mathbb{R}^{N}\right)\right)$ is a weak solution of the following non-linear inhomogeneous parabolic system

$$
v_{t}-\operatorname{div} a(x, t, D v)=\operatorname{div} b(x, t)+f(x, t) \quad \text { in } Q_{R}^{+},
$$

satisfying $v=0$ on the lateral boundary $\Gamma_{R}$ where the vector field $a: Q_{R}^{+} \times \mathbb{R}^{N n} \rightarrow \mathbb{R}^{N n}$ fulfills the following ellipticity and growth conditions:

$$
\langle a(x, t, w) \cdot w\rangle \geqslant v|w|^{2}, \quad|a(x, t, w)| \leqslant L|w|,
$$

for all $(x, t) \in Q_{R}^{+}$and $w \in \mathbb{R}^{N n}$, with $0<v \leqslant 1 \leqslant L$. Then there exists $\sigma_{2}=\sigma_{2}(n, L / v) \in\left(2, \sigma_{1}\right]$ such that Dv $\in$ $L^{\sigma_{2}}\left(Q_{R / 2}^{+} ; \mathbb{R}^{N n}\right)$. Moreover, for any $\sigma \in\left(2, \sigma_{2}\right]$ and $0<\varrho \leqslant R$ and for a constant $c=c(n, N, L / \nu)$, we have

$$
f_{Q_{\varrho / 2}^{+}}|D v|^{\sigma} d z \leqslant c\left(f_{Q_{\varrho}^{+}}|D v|^{2} d z\right)^{\frac{\sigma}{2}}+c f_{Q_{\varrho}^{+}}|b|^{\sigma}+\varrho^{\sigma}|f|^{\sigma} d z .
$$

Proof. First, we will show a reverse-Hölder-type inequality on parabolic cylinders, respectively half-cylinders. We distinguish the following different cases.

Case $z_{0}=\left(x_{0}, t_{0}\right) \in \Gamma_{\boldsymbol{R}}, Q_{2}\left(z_{0}\right) \Subset Q_{\boldsymbol{R}}$. We choose cut-off functions $\eta \in C_{0}^{\infty}\left(B_{\varrho}\left(x_{0}\right)\right)$ with $\eta=1$ on $B_{\varrho / 2}\left(x_{0}\right)$, $0 \leqslant \eta \leqslant 1$ and $|D \eta| \leqslant c / \varrho$ and $\zeta \in C_{0}^{1}\left(\Lambda_{\varrho^{2}}\left(t_{0}\right)\right)$ with $0 \leqslant \zeta \leqslant 1, \zeta=1$ on $\Lambda_{(\varrho / 2)^{2}}\left(t_{0}\right)$ and $\left|\zeta_{t}\right| \leqslant 2 / \varrho^{2}$. Moreover, for $t \in \Lambda_{\varrho^{2}}\left(t_{0}\right), \theta>0$ we define $\chi_{\theta} \in W^{1, \infty}(\mathbb{R})$ as follows: $\chi_{\theta} \equiv 1$ on $(-\infty, t]$, and $\chi_{\theta}(\tau)=1-\frac{1}{\theta}(\tau-t)$ on $(t, t+\theta)$ and $\chi_{\theta} \equiv 0$ on $[t+\theta, \infty)$. We now proceed formally by testing the parabolic system with $\varphi(x, t)=\chi_{\theta}(t) \eta^{2}(x) \zeta(t) v(x, t)$ and then letting $\theta \downarrow 0$. The argument can be justified by use of Steklov averages (see Section 2.2). Testing our parabolic system in its weak formulation we obtain for a.e. $t \in \Lambda_{\varrho^{2}}\left(t_{0}\right)$

$$
\begin{aligned}
& \frac{1}{2} \int_{B_{\varrho}^{+}\left(x_{0}\right)} \eta^{2} \zeta|v(\cdot, t)|^{2} d x+\int_{t_{0}-\varrho^{2}}^{t} \int_{B_{\varrho}^{+}\left(x_{0}\right)} \eta^{2} \zeta\langle a(\cdot, D v), D v\rangle d x d \tau \\
& \quad=\int_{\substack{t_{0}-\varrho^{2} \\
B_{\varrho}^{+}\left(x_{0}\right)}} \eta^{2} \zeta_{t}|v|^{2}-2 \eta \zeta\langle a(\cdot, D v), D \eta \otimes v\rangle-\zeta\left\langle b, D\left(\eta^{2} v\right)\right\rangle-\eta^{2} \zeta\langle f, v\rangle d x d \tau \\
& =: I+I I+I I I+I V,
\end{aligned}
$$

with the obvious meaning of $I-I V$. In the sequel we shall estimate the integrals $I-I V$. Since $\left|\zeta_{t}\right| \leqslant 2 / \varrho^{2}$ we have

$$
|I| \leqslant 2 \int_{Q_{\varrho}^{+}\left(z_{0}\right)}\left|\frac{v}{\varrho}\right|^{2} d z .
$$

Using $|D \eta| \leqslant c / \varrho$, the growth (1.2) 1 of $a$ and Young's inequality with $\varepsilon>0$ (to be chosen later) we find

$$
|I I| \leqslant \varepsilon \int_{t_{0}-\varrho^{2} B_{\varrho}^{+}\left(x_{0}\right)}^{t} \eta^{2} \zeta|D v|^{2} d x d \tau+\frac{c L^{2}}{\varepsilon} \int_{Q_{\varrho}^{+}\left(z_{0}\right)}\left|\frac{v}{\varrho}\right|^{2} d z .
$$

Once again by Young's inequality and the fact that $|D \eta| \leqslant c / \varrho$ we obtain

$$
\begin{aligned}
|I I I| & \leqslant \int_{t_{0}-\varrho^{2}}^{t} \int_{B_{\varrho}^{+}\left(x_{0}\right)}|b|\left(\eta^{2} \zeta|D v|+2 \eta \zeta|D \eta||v|\right) d x d \tau \\
& \leqslant \varepsilon \int_{t_{0}-\varrho^{2} B_{\varrho}^{+}\left(x_{0}\right)}^{t} \eta^{2} \zeta|D v|^{2} d x d \tau+\varepsilon \int_{Q_{\varrho}^{+}\left(z_{0}\right)}\left|\frac{v}{\varrho}\right|^{2} d z+\frac{c}{\varepsilon} \int_{Q_{\varrho}^{+}\left(z_{0}\right)}|b|^{2} d z,
\end{aligned}
$$


and similarly

$$
|I V| \leqslant \int_{Q_{\varrho}^{+}\left(z_{0}\right)}|f \| v| d z \leqslant \int_{Q_{\varrho}^{+}\left(z_{0}\right)}\left|\frac{v}{\varrho}\right|^{2} d z+\varrho^{2} \int_{Q_{\varrho}^{+}\left(z_{0}\right)}|f|^{2} d z .
$$

Moreover, with the help of the ellipticity assumption on the vector field $a$ we can bound the second integral on the left-hand side of (3.1) from above by

$$
v \int_{t_{0}-\varrho^{2}}^{t} \int_{B_{\varrho}^{+}\left(x_{0}\right)} \eta^{2} \zeta|D v|^{2} d x d \tau
$$

Choosing $\varepsilon$ small enough (i.e. $\varepsilon=v / 4$ ) in order to absorb the terms involving $D v$ on the left-hand side we get

$$
\int_{B_{\varrho}^{+}\left(x_{0}\right)} \eta^{2} \zeta|v(\cdot, t)|^{2} d x+\int_{t_{0}-\varrho^{2}}^{t} \int_{B_{\varrho}^{+}\left(x_{0}\right)} \eta^{2} \zeta|D v|^{2} d x d \tau \leqslant c \int_{Q_{\varrho}^{+}\left(z_{0}\right)}\left|\frac{v}{\varrho}\right|^{2}+|b|^{2}+\varrho^{2}|f|^{2} d z,
$$

for a.e. $t \in \Lambda_{Q^{2}}\left(t_{0}\right)$ where $c=c(L / v)$ (note that we have assumed $v \leqslant 1 \leqslant L$ ). Taking the supremum over $t \in \Lambda_{\varrho^{2}}\left(t_{0}\right)$ in the first term of the left-hand side as well as letting $t=t_{0}+\varrho^{2}$ in the second one, noting that $\eta \equiv 1$ on $B_{\varrho / 2}^{+}\left(x_{0}\right)$ and $\zeta \equiv 1$ on $\Lambda_{(\varrho / 2)^{2}}\left(t_{0}\right)$ and taking mean values we finally arrive at the following Caccioppoli inequality

$$
\sup _{t \in \Lambda_{(\varrho / 2)^{2}}\left(t_{0}\right)} f_{B_{\varrho / 2}^{+}\left(x_{0}\right)}\left|\frac{v(\cdot, t)}{\varrho}\right|^{2} d x+f_{Q_{\varrho / 2}^{+}\left(x_{0}\right)}|D v|^{2} d z \leqslant c f_{Q_{\varrho}^{+}\left(z_{0}\right)}\left|\frac{v}{\varrho}\right|^{2}+|b|^{2}+\varrho^{2}|f|^{2} d z .
$$

We note that this inequality holds for any parabolic cylinder with $z_{0} \in \Gamma_{R}$ and $Q_{\varrho}\left(z_{0}\right) \subset Q_{R}$. Therefore, starting with a cylinder $Q_{\varrho}\left(z_{0}\right)$ such that $Q_{2 \varrho}\left(z_{0}\right) \Subset Q_{R}$ we can apply the preceding inequality with $Q_{\varrho}^{+}\left(z_{0}\right)$ replaced by $Q_{2 \varrho}^{+}\left(z_{0}\right)$. Moreover, since $v=0$ on $\Gamma_{\varrho}\left(z_{0}\right)$ we can apply the Sobolev-Poincaré inequality slice wise on $B_{\varrho}^{+}\left(x_{0}\right) \times\{t\}$. Proceeding this way leads us to

$$
\begin{aligned}
f_{Q^{+}\left(z_{0}\right)}\left|\frac{v}{\varrho}\right|^{2} d z & \leqslant\left[\left.\sup _{t \in \Lambda_{\varrho^{2}\left(t_{0}\right)}} f\left|\frac{v(\cdot, t)}{\varrho}\right|^{2}\right|^{+} d x\right]^{\frac{2}{n+2}\left(x_{0}\right)} \underset{\Lambda_{\varrho^{2}}\left(t_{0}\right)}{f}\left[f_{B_{\varrho}^{+}\left(x_{0}\right)}\left|\frac{v}{\varrho}\right|^{2} d x\right]^{\frac{n}{n+2}} d \tau \\
& \leqslant c\left[f_{Q_{2 \varrho}^{+}\left(z_{0}\right)}\left|\frac{v}{\varrho}\right|^{2}+|b|^{2}+\varrho^{2}|f|^{2} d z\right]^{\frac{2}{n+2}} f_{Q_{\varrho}^{+}\left(z_{0}\right)}|D v|^{\frac{2 n}{n+2}} d z .
\end{aligned}
$$

We further estimate the right-hand side by Young's inequality with $\varepsilon>0$ (to be chosen later) and Poincaré's inequality (which can be applied slice wise to $|v(\cdot, t)|^{2}$ for a.e. $t \in \Lambda_{\varrho^{2}}\left(t_{0}\right)$ since $v=0$ on $\Gamma_{\varrho}\left(z_{0}\right)$ ) to obtain

$$
f_{Q_{\varrho}^{+}\left(z_{0}\right)}\left|\frac{v}{\varrho}\right|^{2} d z \leqslant+\varrho^{2}|f|^{2} d z+\frac{c}{\varepsilon}\left[f_{Q_{\varrho}^{+}\left(z_{0}\right)}|D v|^{\frac{2 n}{n+2}} d z\right]^{\frac{n+2}{n}},
$$

where $c=c(n, L / v)$. Using this estimate to bound the term involving $\left|\frac{v}{\varrho}\right|^{2}$ in the above Caccioppoli inequality and choosing $\varepsilon>0$ suitably small (i.e. $\varepsilon=1 /(2 c)$ ) we arrive at the following reverse-Hölder-type inequality:

$$
f_{Q_{\varrho / 2}^{+}\left(x_{0}\right)}|D v|^{2} d z \leqslant \frac{1}{2} f_{Q_{2 \varrho}^{+}\left(z_{0}\right)}|D v|^{2} d z+c\left[f_{Q_{\varrho}^{+}\left(z_{0}\right)}|D v|^{\frac{2 n}{n+2}} d z\right]^{\frac{n+2}{n}}+c f_{Q_{2 \varrho}^{+}\left(z_{0}\right)}|b|^{2}+\varrho^{2}|f|^{2} d z,
$$

where $c=c(n, L / v)$. 
Case $\boldsymbol{Q}_{2 \varrho}\left(z_{0}\right) \subset \boldsymbol{Q}_{\boldsymbol{R}}^{+}$. Here, we test the weak formulation of the parabolic system with the test-function $\varphi(x, t)=$ $\chi_{\theta}(t) \eta^{2}(x) \zeta(t)\left(v(x, t)-(v)_{\eta}(t)\right)$ where

$$
(v)_{\eta}(t)=\left(\int_{B_{\varrho}\left(x_{0}\right)} \eta^{2}(x) d x\right)^{-1} \int_{B_{\varrho}\left(x_{0}\right)} v(x, t) \eta^{2}(x) d x .
$$

The definition of $(v)_{\eta}$ easily yields

$$
\int_{t_{0}-\varrho^{2}}^{t} \int_{B_{\varrho}\left(x_{0}\right)} \partial_{t}(v)_{\eta} \zeta \eta^{2}\left(v-(v)_{\eta}\right) d x d \tau=0 .
$$

Therefore, with the arguments from the first case we can deduce the following reverse-Hölder inequality for interior cylinders:

$$
f_{Q_{\varrho / 2}\left(x_{0}\right)}|D v|^{2} d z \leqslant \frac{1}{2} f_{Q_{2 \varrho}\left(z_{0}\right)}|D v|^{2} d z+c\left[f_{Q_{\varrho}\left(z_{0}\right)}|D v|^{\frac{2 n}{n+2}} d z\right]^{\frac{n+2}{n}}+c f_{Q_{2 \varrho}\left(z_{0}\right)}|b|^{2}+\varrho^{2}|f|^{2} d z,
$$

where $c=c(n, L / v)$. We note that by a different choice of the cut-off functions we can attain on the right-hand side of the reverse-Hölder inequalities above integrals involving only $Q_{\varrho}^{+}\left(z_{0}\right)$, respectively $Q_{\varrho}\left(z_{0}\right)$ instead of $Q_{2 \varrho}^{+}\left(z_{0}\right)$, respectively $Q_{2 \varrho}\left(z_{0}\right)^{+}$. Hence, we are exactly in a position to apply the up to the boundary version of Gehring's theorem from [14, Theorem 2.4] which extends verbatim to the case when we are considering parabolic cylinders (with slight modifications allowing for the presence of the first integral on the right-hand side of the reverse-Hölder inequality, see for instance [22, Chapter V] or [23, Proposition 1.1, Theorem 6.6]). The application of Gehring's theorem then yields the existence of a higher integrability exponent $\sigma_{2}=\sigma_{2}(n, L / v) \in\left(2, \sigma_{1}\right]$ such that $D v \in L^{\sigma_{2}}\left(Q_{R / 2}^{+} ; \mathbb{R}^{N n}\right)$. Moreover, for any $0<\varrho \leqslant R$ and $\sigma \in\left(2, \sigma_{2}\right]$ we have

$$
f_{Q_{\varrho / 2}^{+}}|D v|^{\sigma} d z \leqslant c\left(f_{Q_{\varrho}^{+}}|D v|^{2} d z\right)^{\frac{\sigma}{2}}+c f_{Q_{\varrho}^{+}}|b|^{\sigma}+\varrho^{\sigma}|f|^{\sigma} d z,
$$

where $c=c(n, N, L / v)$.

\section{Differentiable parabolic systems}

In this section we are concerned with the up to the boundary higher differentiability properties of weak solutions of certain non-linear parabolic systems with Lipschitz continuous coefficients of linear growth. To be precise, on the half-cylinder $Q_{R}^{+}$, respectively on $Q_{R}^{0}, R>0$, we consider a weak solution $u$ of the following parabolic system

$$
u_{t}-\operatorname{div} a(x, t, D u)=G(x, t) \quad \text { in } Q \text {, }
$$

where either $Q=Q_{R}^{+}$or $Q=Q_{R}^{0}$. In the lateral boundary situation we consider weak solutions $u \in L^{2}\left(\Lambda_{R^{2}}\right.$; $\left.W^{1,2}\left(B_{R}^{+} ; \mathbb{R}^{N}\right)\right)$, satisfying $u=0$ on $\Gamma_{R}$, whereas in the initial boundary situation we consider $u \in L^{2}\left(\Lambda_{R^{2}}^{0}\right.$; $W^{1,2}\left(B_{R} ; \mathbb{R}^{N}\right)$ ), satisfying $u(\cdot, 0)=0$ on $B_{R}$ and, moreover $G=0$. For the vector field $a: Q_{R}^{+} \times \mathbb{R}^{N n} \rightarrow \mathbb{R}^{N n}$, respectively $a: Q_{R}^{0} \times \mathbb{R}^{N n} \rightarrow \mathbb{R}^{N n}$ we assume that $a$ is differentiable with respect to $w$, Lipschitz continuous with respect to $x$, Hölder continuous with respect to $t$ and $(x, t, w) \mapsto a(x, t, w), \partial_{w} a(x, t, w)$ are Carathéodory functions. Moreover, we shall assume the following ellipticity and growth conditions on $a$ :

$$
\left\{\begin{array}{l}
|a(x, t, w)|+(1+|w|)\left|\partial_{w} a(x, t, w)\right| \leqslant L(1+|w|), \\
\left\langle\partial_{w} a(x, t, w) \tilde{w},\left.\tilde{w}|\geqslant v| \tilde{w}\right|^{2},\right. \\
|a(x, t, w)-a(\tilde{x}, t, w)| \leqslant L \gamma|x-\tilde{x}|(1+|w|), \\
|a(x, t, w)-a(x, \tau, w)| \leqslant L \gamma|t-\tau|^{\frac{1+\vartheta}{2}}(1+|w|),
\end{array}\right.
$$

for all $x, \tilde{x} \in B_{R}^{+}$, respectively $B_{R}$ and $t, \tau \in \Lambda_{R^{2}}$, respectively $\Lambda_{R^{2}}^{0}$ and $w, \tilde{w} \in \mathbb{R}^{N n}$, where $0<v \leqslant 1 \leqslant L<\infty$, $\gamma \geqslant 1$ and $\vartheta \in(0,1)$. 


\subsection{The lateral boundary}

We first consider the lateral boundary situation, where $Q=Q_{R}^{+}$in (4.1). Throughout the whole section we shall consider weak solutions under the following general assumptions:

Definition 4.1. By a weak solution of the parabolic system (4.1) on $Q_{R}^{+}$we mean a function $u \in L^{2}\left(\Lambda_{R^{2}}\right.$; $\left.W^{1,2}\left(B_{R}^{+} ; \mathbb{R}^{N}\right)\right)$ with $u=0$ on the lateral boundary $\Gamma_{R}$ which satisfies (4.1) in the weak sense. Moreover, the structure conditions (4.2) are in force.

As usual, we can rewrite the weak formulation of the parabolic system equivalently using the Steklov-means defined in (2.4) - see also [7, Section 2.2] - i.e. for a.e. $t \in \Lambda_{R^{2}}$ and $0<|h| \leqslant T / 2$ there holds

$$
\int_{B_{R}^{+}} \partial_{t} u_{h}(\cdot, t) \cdot \varphi+\left\langle[a(\cdot, t, D u(\cdot, t))]_{h}, D \varphi\right\rangle d x=\int_{B_{R}^{+}}\left\langle G_{h}(\cdot, t), \varphi\right\rangle d x,
$$

for all $\varphi \in C_{0}^{\infty}\left(B_{R}^{+} ; \mathbb{R}^{N}\right)$. See $[7$, Section 2.2] for related definitions and notations.

\subsubsection{Higher differentiability}

Let us recall the interior higher differentiability result from [18, Lemmas 5.1 and 5.2] stating that $u$ admits a second spatial derivative $D^{2} u \in L_{\text {loc }}^{2}$ and a first time-derivative $\partial_{t} u \in L_{\text {loc }}^{2}$ in the interior. Our aim here is to extend these higher differentiability properties up to the lateral boundary $\Gamma$. Let us mention that neither the proof for the parabolic interior situation nor the one for the elliptic boundary situation does directly apply here. The basic difficulty is the following, and it has been already mentioned in the Introduction: As usual we start proving that the second tangential derivatives $D_{\alpha} D_{\beta} u, \alpha+\beta<2 n$ exist in $L^{2}$. But then we are left with two missing derivatives to estimate, namely the first time derivatives, and the second normal derivatives, but for no more than one of them we can exploit the parabolic system. In the elliptic situation this difficulty does not arise - see for instance [26, Section 4] and the references therein - in fact the only missing direction is the normal one and estimates for it can be obtained starting from the estimates for the tangential direction and the fact that the normal direction solves a differentiated form of the systems in the interior of the domain. To overcome this difficulty we shall use an iterated finite difference method which employs estimates for fractional second finite differences in time to finally achieve a full first derivative in time of the solution. The iteration scheme can be roughly illustrated as follows:

$$
\Delta_{h}^{\frac{1}{2}} u \Rightarrow \Delta_{h}^{\frac{1}{2}} D u \Rightarrow \Delta_{h}^{\frac{1}{2}} \Delta_{h}^{\frac{1}{2}} u \Rightarrow \Delta_{h}^{1-\frac{\vartheta}{4}} u \Rightarrow \Delta_{h}^{\frac{1}{2}+\frac{\vartheta}{4}} D u \Rightarrow \Delta_{h}^{\frac{1}{2}} \Delta_{h}^{\frac{1}{2}+\frac{\vartheta}{4}} u \Rightarrow \Delta_{h}^{1} u .
$$

The preceding inclusions have to be understood as follows: all the implications mean that the quantities considered are uniformly bounded - with respect to the difference parameter $h>0$ - in the $L^{2}$-norm, while $\Delta_{h}^{\alpha}=h^{-\alpha} \tau_{h}$, see (2.6); recall also from $(4.2)_{4}$ that $t \mapsto a(x, t, w) \in C^{0,(1+\vartheta) / 2}$, with $\vartheta \in(0,1)$. The $L^{2}$-estimate for $\Delta_{h}^{1} u$ then yields a time derivative $\partial_{t} u \in L^{2}$. This iteration will be performed through Lemma 4.4 - Proposition 4.8. Then, having proved the existence of the first time derivative in $L^{2}$ we can finally exploit the system for the second normal derivative, yielding that we also have $D_{n} D_{n} u \in L^{2}$. Since the result seems to be interesting in its own we shall summarize it in the following theorem which is a consequence of Propositions 4.3, 4.8 and 4.10 below.

Theorem 4.2. Suppose that $u \in L^{2}\left(\Lambda_{R^{2}} ; W^{1,2}\left(B_{R}^{+} ; \mathbb{R}^{N}\right)\right)$ with $u=0$ on the lateral boundary $\Gamma_{R}$ is a weak solution of the parabolic system (4.1) where the structure conditions (4.2) are in force. Moreover, let $G \in \mathcal{N}^{0, \vartheta ; 2}\left(Q_{R}^{+} ; \mathbb{R}^{N}\right)$, where $\vartheta$ has been defined in (4.2) 4 . Then, $D^{2} u \in L^{2}\left(Q_{r}^{+}, \mathbb{R}^{n^{2} N}\right)$ and $\partial_{t} u \in L^{2}\left(Q_{r}^{+}, \mathbb{R}^{N}\right)$ for all $0<r<R$. Moreover, for any $0<\varrho \leqslant R$ there holds

$$
\int_{Q_{\varrho / 2}^{+}}\left|D^{2} u\right|^{2}+\left|\partial_{t} u\right|^{2} d z \leqslant c\left[\left(\gamma^{2}+\varrho^{-2}\right) \int_{Q_{\varrho}^{+}}\left(1+|D u|^{2}\right) d z+\|G\|_{\mathcal{N}^{0, \vartheta ; 2}\left(Q_{\varrho}^{+}\right)}^{2}\right],
$$

where $c=c(n, N, L / v, \vartheta)$ and $c \rightarrow \infty$ when $\vartheta \downarrow 0$. 
As mentioned above we first consider the derivatives $D_{\alpha} D u$ with respect to the tangential directions $\alpha=$ $1, \ldots, n-1$. We start with a Caccioppoli-type inequality for the tangential derivatives.

Proposition 4.3. Let u be a weak solution of (4.1) on $Q_{R}^{+}$according to Definition 4.1. Then, Du is weakly differentiable with respect to $x_{\alpha}$ for any $\alpha=1, \ldots, n-1$ and $D_{\alpha} D u \in L^{2}\left(Q_{r}^{+}, \mathbb{R}^{N n}\right)$ for all $0<r<R$. Moreover, there exists a constant $c=c(n, N, L / v)$ such that for any $0<\varrho \leqslant R$ we have

$$
\sup _{t \in \Lambda_{(\varrho / 2)^{2}}} \int_{B_{\varrho / 2}^{+}}\left|D_{\alpha} u(\cdot, t)\right|^{2} d x+\int_{Q_{\varrho / 2}^{+}}\left|D_{\alpha} D u\right|^{2} d z \leqslant c\left[\left(\gamma^{2}+\varrho^{-2}\right) \int_{Q_{\varrho}^{+}}\left(1+|D u|^{2}\right) d z+\int_{Q_{\varrho}^{+}}|G|^{2} d z\right] .
$$

Proof. We consider a fixed tangential direction $x_{\alpha}, \alpha=1, \ldots, n-1$. For $|h|$ sufficiently small we replace in (4.3) (after replacing $h$ by $\lambda$ ) the test-function $\varphi$ by $\tau_{-h}^{\alpha} \varphi$, perform an integration by parts for finite differences and then integrate for $t \in \Lambda_{\varrho^{2}}$ over $\left(-\varrho^{2}, t\right)$. This leads us to

$$
\int_{-\varrho^{2}}^{t} \int_{B_{R}^{+}} \partial_{t} \tau_{h}^{\alpha}\left[u_{\lambda}\right] \cdot \varphi+\left\langle\tau_{h}^{\alpha}[a(\cdot, D u)]_{\lambda}, D \varphi\right\rangle d x d \tau=\int_{-\varrho^{2}}^{t} \int_{B_{R}^{+}}\left\langle G_{\lambda}, \tau_{-h}^{\alpha} \varphi\right\rangle d x d \tau
$$

Now, we choose cut-off functions $\eta \in C_{0}^{\infty}\left(B_{3 \varrho / 4}\right)$ with $0 \leqslant \eta \leqslant 1, \eta \equiv 1$ on $B_{\varrho / 2},|D \eta| \leqslant c / \varrho$ and $\zeta \in C^{1}(\mathbb{R})$ with $0 \leqslant \zeta \leqslant 1, \zeta \equiv 0$ on $\left(-\infty,-\varrho^{2}\right), \zeta \equiv 1$ on $\left(-(\varrho / 2)^{2}, \infty\right)$ and $\left|\zeta^{\prime}\right| \leqslant 2 / \varrho^{2}$. Choosing in the preceding identity the test-function $\varphi(x, t)=\eta^{2}(x) \zeta(t) \tau_{h}^{\alpha}\left[u_{\lambda}\right](x, t)$ with $0<|h| \leqslant \varrho / 8$ the first term on the right-hand side can be rewritten in the form

$$
\int_{-\varrho^{2}}^{t} \int_{B_{R}^{+}} \partial_{t} \tau_{h}^{\alpha}\left[u_{\lambda}\right] \cdot \varphi d x d \tau=\frac{1}{2} \int_{B_{\varrho}^{+}}\left|\tau_{h}^{\alpha} u_{\lambda}(\cdot, t)\right|^{2} \eta^{2} \zeta(t) d x-\frac{1}{2} \int_{-\varrho^{2} B_{\varrho}^{+}}^{t}\left|\tau_{h}^{\alpha} u_{\lambda}\right|^{2} \eta^{2} \zeta_{t} d x d \tau .
$$

Passing to the limit $\lambda \downarrow 0$ we therefore obtain

$$
\begin{aligned}
& \frac{1}{2} \int_{B_{\varrho}^{+}}\left|\tau_{h}^{\alpha} u(\cdot, t)\right|^{2} \eta^{2} \zeta(t) d x+\int_{-\varrho^{2} B_{\varrho}^{+}}^{t}\left\langle\tau_{h}^{\alpha}[a(\cdot, D u)], D \tau_{h}^{\alpha} u\right\rangle \eta^{2} \zeta d x d \tau \\
& \quad=\int_{-\varrho^{2}}^{t} \int_{B_{\varrho}^{+}} \frac{1}{2}\left|\tau_{h}^{\alpha} u\right|^{2} \eta^{2} \zeta_{t}-2\left\langle\tau_{h}^{\alpha}[a(\cdot, D u)], D \eta \otimes \tau_{h}^{\alpha} u\right\rangle \eta \zeta+\left\langle G, \tau_{-h}^{\alpha}\left(\eta^{2} \tau_{h}^{\alpha} u\right)\right\rangle \zeta d x d \tau,
\end{aligned}
$$

for a.e. $t \in \Lambda_{\varrho^{2}}$. We now decompose

$$
\tau_{h}^{\alpha}[a(\cdot, \cdot, D u(\cdot, \cdot))](x, t)=\mathcal{A}(h)(x, t)+\mathcal{B}(h)(x, t),
$$

where

$$
\begin{aligned}
\mathcal{A}(h)(x, t) & :=a\left(x+h e_{\alpha}, t, D u\left(x+h e_{\alpha}, t\right)\right)-a\left(x+h e_{\alpha}, t, D u(x, t)\right) \\
& =\int_{0}^{1} \partial_{w} a\left(x+h e_{\alpha}, t, D u(x, t)+s \tau_{h}^{\alpha}[D u](x, t)\right) d s \tau_{h}^{\alpha}[D u](x, t)
\end{aligned}
$$

and $\mathcal{B}(h)(x, t):=a\left(x+h e_{\alpha}, t, D u(x, t)\right)-a(x, t, D u(x, t))$. Then the preceding integral identity can be rewritten as follows:

$$
\frac{1}{2} \int_{B_{\varrho}^{+}}\left|\tau_{h}^{\alpha} u(\cdot, t)\right|^{2} \eta^{2} \zeta(t) d x+\int_{-\varrho^{2}}^{t} \int_{B_{\varrho}^{+}}\left\langle\mathcal{A}(h)+\mathcal{B}(h), D \tau_{h}^{\alpha} u\right| \eta^{2} \zeta d x d \tau
$$




$$
=-2 \int_{-\varrho^{2}}^{t} \int_{B_{\varrho}^{+}}\left\langle\mathcal{A}(h)+\mathcal{B}(h), D \eta \otimes \tau_{h}^{\alpha} u\right\rangle \eta \zeta d x d \tau+\int_{-\varrho^{2}}^{t} \int_{B_{\varrho}^{+}} \frac{1}{2}\left|\tau_{h}^{\alpha} u\right|^{2} \eta^{2} \zeta_{t}+\left\langle G, \tau_{-h}^{\alpha}\left(\eta^{2} \tau_{h}^{\alpha} u\right)\right\rangle \zeta d x d \tau .
$$

From the ellipticity condition $(4.2)_{2}$ we infer that

$$
\left\langle\mathcal{A}(h)(x, t), D \tau_{h}^{\alpha} u(x, t)\right\rangle \geqslant v\left|\tau_{h}^{\alpha}[D u](x, t)\right|^{2} .
$$

This implies for the integral involving $\mathcal{A}(h)$ on the left-hand side of (4.6) that

$$
\int_{-\varrho^{2} B_{\varrho}^{+}}^{t}\left\langle\mathcal{A}(h),\left.D \tau_{h}^{\alpha} u\left|\eta^{2} \zeta d x d \tau \geqslant v \int_{-\varrho^{2}}^{t} \int_{B_{\varrho}^{+}}\right| \tau_{h}^{\alpha}[D u]\right|^{2} \eta^{2} \zeta d x d \tau\right.
$$

On the other hand, from the upper bound (4.2) 1 we have $|\mathcal{A}(h)(x, t)| \leqslant L\left|\tau_{h}^{\alpha}[D u](x, t)\right|$. Hence, the integral involving $\mathcal{A}(h)$ on the right-hand side of (4.6) can be estimated using Young's inequality with $\varepsilon>0$ (to be chosen later) by

$$
\begin{aligned}
2 \mid \int_{-\varrho^{2}} \int_{B_{\varrho}^{+}}\left\langle\mathcal{A}(h), D \eta \otimes \tau_{h}^{\alpha} u|\eta \zeta d x d \tau|\right. & \leqslant 2 L \int_{-\varrho^{2}}^{t} \int_{\varrho}\left|\tau_{h}^{\alpha}[D u]\right||D \eta|\left|\tau_{h}^{\alpha} u\right| \eta \zeta d x d \tau \\
& \leqslant \varepsilon \int_{-\varrho^{2}}^{t} \int_{B_{\varrho}^{+}}\left|\tau_{h}^{\alpha}[D u]\right|^{2} \eta^{2} \zeta d x d \tau+\frac{L^{2}}{\varepsilon} \int_{-\varrho^{2}}^{t} \int_{B_{\varrho}^{+}}|D \eta|^{2}\left|\tau_{h}^{\alpha} u\right|^{2} \zeta d x d \tau .
\end{aligned}
$$

To estimate the integrals involving $\mathcal{B}(h)$ in (4.6) we exploit the Lipschitz continuity of the vector field $a(x, t, w)$ with respect to $x$ from $(4.2)_{3}$ in the form of the inequality $|\mathcal{B}(h)(x, t)| \leqslant L \gamma|h|(1+|D u(x, t)|)$. Subsequently using Young's inequality we obtain

$$
\left|\int_{-\varrho^{2}}^{t} \int_{B_{\varrho}^{+}}\left\langle\mathcal{B}(h), D \tau_{h}^{\alpha} u\right\rangle \eta^{2} \zeta d x d \tau\right| \leqslant \varepsilon \int_{-\varrho^{2}}^{t} \int_{B_{\varrho}^{+}}\left|\tau_{h}^{\alpha}[D u]\right|^{2} \eta^{2} \zeta d x d \tau+\frac{L^{2} \gamma^{2}|h|^{2}}{\varepsilon} \int_{-\varrho^{2}}^{t} \int_{B_{\varrho}^{+}}(1+|D u|)^{2} \eta^{2} \zeta d x d \tau
$$

and similarly

$$
\begin{aligned}
& 2 \mid \int_{-\varrho^{2}}^{t} \int_{B_{\varrho}^{+}}\left\langle\mathcal{B}(h), D \eta \otimes \tau_{h}^{\alpha} u|\eta \zeta d x d \tau|\right. \\
& \quad \leqslant \varepsilon \int_{-\varrho^{2}}^{t} \int_{B_{\varrho}^{+}}|D \eta|^{2}\left|\tau_{h}^{\alpha} u\right|^{2} \zeta d x d \tau+\frac{L^{2} \gamma^{2}|h|^{2}}{\varepsilon} \int_{-\varrho^{2}}^{t} \int_{B_{\varrho}^{+}}(1+|D u|)^{2} \eta^{2} \zeta d x d \tau .
\end{aligned}
$$

We now turn our attention to the estimate of the integral containing the inhomogeneity $G$. First, we write

$$
\begin{aligned}
\int_{-\varrho^{2} B_{\varrho}^{+}}^{t} \int_{1}\left\langle G, \tau_{-h}^{\alpha}\left(\eta^{2} \tau_{h}^{\alpha} u\right)\right\rangle \zeta d x d \tau & =\int_{-\varrho^{2} B_{\varrho}^{+}}^{t} \int_{l}\left\langle G, \eta\left(x-h e_{\alpha}\right) \tau_{-h}^{\alpha}\left(\eta \tau_{h}^{\alpha} u\right)\right\rangle \zeta+\left\langle G,\left(\tau_{-h}^{\alpha} \eta\right)\left(\eta \tau_{h}^{\alpha} u\right)\right\rangle \zeta d x d \tau \\
& =: I+I I,
\end{aligned}
$$

with the obvious meaning of $I$ and $I I$. In turn we estimate $I$ with Young's inequality and (2.7) by

$$
|I| \leqslant \int_{-\varrho^{2}}^{t} \int_{B_{\varrho}^{+}}|G|\left|\tau_{-h}^{\alpha}\left(\eta \tau_{h}^{\alpha} u\right)\right| \zeta d x d \tau
$$




$$
\begin{aligned}
& \leqslant \frac{\varepsilon}{h^{2}} \int_{-\varrho^{2}}^{t} \int_{B_{\varrho}^{+}}\left|\tau_{-h}^{\alpha}\left(\eta \tau_{h}^{\alpha} u\right)\right|^{2} \zeta d x d \tau+\frac{h^{2}}{\varepsilon} \int_{Q_{\varrho}^{+}}|G|^{2} d z \\
& \leqslant \varepsilon \int_{-\varrho^{2}}^{t} \int_{B_{\varrho}^{+}}\left|D_{\alpha}\left(\eta \tau_{h}^{\alpha} u\right)\right|^{2} \zeta d x d \tau+\frac{h^{2}}{\varepsilon} \int_{Q_{\varrho}^{+}}|G|^{2} d z \\
& \leqslant c \varepsilon \int_{-\varrho^{2}}^{t} \int_{B_{\varrho}^{+}}\left|\tau_{h}^{\alpha} D u\right|^{2} \eta^{2} \zeta+|D \eta|^{2}\left|\tau_{h}^{\alpha} u\right|^{2} \zeta d x d \tau+\frac{h^{2}}{\varepsilon} \int_{Q_{\varrho}^{+}}|G|^{2} d z .
\end{aligned}
$$

Using again Young's inequality and the fact that $|D \eta| \leqslant c / \varrho$ we get

$$
\begin{aligned}
|I I| & \leqslant \int_{Q_{\varrho}^{+}}|G|\left|\tau_{-h}^{\alpha} \eta\right|\left|\tau_{h}^{\alpha} u\right| \eta \zeta d z \\
& \leqslant \varrho^{-2} \int_{Q_{\varrho}^{+}}\left|\tau_{h}^{\alpha} u\right|^{2} \eta^{2} \zeta d z+\varrho^{2} \int_{Q_{\varrho}^{+}}|G|^{2}\left|\tau_{-h}^{\alpha} \eta\right|^{2} d z \\
& \leqslant \varrho^{-2} \int_{Q_{\varrho}^{+}}\left|\tau_{h}^{\alpha} u\right|^{2} \eta^{2} \zeta d z+\varrho^{2} h^{2} \int_{Q_{\varrho}^{+}}|G(x, t)|^{2} \int_{0}^{1}\left|D \eta\left(x-s h e_{\alpha}\right)\right|^{2} d s d z \\
& \leqslant \varrho^{-2}\left|\tau_{h}^{\alpha} u\right|^{2} \eta^{2} \zeta d z+c h^{2} \int_{Q_{\varrho}^{+}}|G|^{2} d z .
\end{aligned}
$$

Joining the preceding estimates with (4.6), noting that $\left|\zeta_{t}\right| \leqslant 2 / \varrho^{2},|D \eta| \leqslant c / \varrho$, spt $\eta \subset B_{3 \varrho / 4}$ and choosing $\varepsilon$ sufficiently small in order to re-absorb the integrals involving $\left|\tau_{h}^{\alpha}[D u]\right|$ we arrive at

$$
\begin{aligned}
& \int_{B_{\varrho}^{+}}\left|\tau_{h}^{\alpha} u(\cdot, t)\right|^{2} \eta^{2} \zeta(t) d x+\int_{-\varrho^{2}}^{t} \int_{B_{\varrho}^{+}}\left|\tau_{h}^{\alpha}[D u]\right|^{2} \eta^{2} \zeta d x d \tau \\
& \quad \leqslant c \varrho^{-2} \int_{Q_{3 \varrho / 4}^{+}}\left|\tau_{h}^{\alpha} u\right|^{2} d z+c h^{2} \int_{Q_{\varrho}^{+}}\left(\gamma^{2}(1+|D u|)^{2}+|G|^{2}\right) d z,
\end{aligned}
$$

where $c=c(n, L / v)$. Passing in the first term on the left-hand side to the supremum over $t \in \Lambda_{\varrho^{2}}$, taking $t=\varrho^{2}$ in the second one, noting that $\eta=1$ on $B_{\varrho / 2}$ and $\zeta=1$ on $\Lambda_{(\varrho / 2)^{2}}$ and finally using Young's inequality, (2.7) and the fact that $0<|h| \leqslant \varrho / 8$ leads us to

$$
\sup _{t \in \Lambda_{(\varrho / 2)^{2}}} \int_{B_{\varrho / 2}^{+}}\left|\tau_{h}^{\alpha} u(\cdot, t)\right|^{2} d x+\int_{Q_{\varrho / 2}^{+}}\left|\tau_{h}^{\alpha}[D u]\right|^{2} d z \leqslant c h^{2}\left[\left(\gamma^{2}+\varrho^{-2}\right) \int_{Q_{\varrho}^{+}}\left(1+|D u|^{2}\right) d z+\int_{Q_{\varrho}^{+}}|G|^{2} d z\right] .
$$

Since $0<|h| \leqslant \varrho / 8$ was arbitrary this proves that $D u$ is weakly differentiable with respect to $x_{\alpha}$. Moreover, the desired Caccioppoli-type estimate holds. Note that by a different choice of the involved radii and cylinder we can infer that $D_{\alpha} D u \in L^{2}\left(Q_{r}^{+}, \mathbb{R}^{N n}\right)$ for any $0<r<R$. This finishes the proof of the lemma.

Our next aim is to show that $u$ is weakly differentiable with respect to time. In the interior this can be achieved by using the system and the weak differentiability of $D u$ with respect to $x$ (see for instance [2,6,18]). In order to exploit the system we have to control either the time derivative $\partial_{t} u$ or $D_{n} D u$ up to the boundary $\Gamma_{R}$. As described at the beginning of the section we shall derive an $L^{2}$-estimate for $\partial_{t} u$ up to the boundary by a reiteration technique for finite differences and then exploit the parabolic system in order to control $D_{n} D u$. We start with the following 
Lemma 4.4. Let $u$ be a weak solution of (4.1) on $Q_{R}^{+}$according to Definition 4.1. Then, for all $0<r<\varrho \leqslant R$ and $0<|h| \leqslant \min \left\{(\varrho-r)^{2}, R^{2}-\varrho^{2}\right\}$ there holds

$$
\int_{Q_{r}^{+}} \frac{\left|\tau_{h} u\right|^{2}}{|h|} d z \leqslant c(L) \int_{Q_{\varrho}^{+}}\left(1+|D u|^{2}+\varrho^{2}|G|^{2}\right) d z
$$

Proof. We choose a cut-off function $\eta \in C_{0}^{\infty}\left(B_{\varrho}\right)$ with $0 \leqslant \eta \leqslant 1, \eta \equiv 1$ on $B_{r}$ and $|D \eta| \leqslant 2 /(\varrho-r)$. Taking $\varphi(x, t)=\eta^{2}(x) \tau_{h} u(x, t)$ as test-function in the Steklov-formulation (4.3) of the parabolic system, integrating with respect to $t$ over $\Lambda_{r^{2}}$ and recalling the identity $\tau_{h} u=|h| \partial_{t} u_{h}$ we find

$$
\begin{aligned}
\int_{\Lambda_{r^{2}} \int_{B^{+}}} \frac{\left|\tau_{h} u\right|^{2}}{|h|} \eta^{2} d x d t & =\iint_{\Lambda_{r^{2}} B_{\varrho}^{+}} \partial_{t} u_{h} \cdot \eta^{2} \tau_{h} u d x d t \\
& =-\int_{\Lambda_{r^{2}}} \int_{B_{\varrho}^{+}}\left(\left\langle[a(\cdot, D u)]_{h}, D\left(\eta^{2} \tau_{h} u\right)\right\rangle-\left\langle G_{h}, \eta^{2} \tau_{h} u\right\rangle\right) d x d t .
\end{aligned}
$$

Using the bound from $(4.2)_{1}$ on the coefficients $a$, the facts that $|D \eta| \leqslant 2 /(\varrho-r) \leqslant 2 / \sqrt{|h|},|h| \leqslant \varrho^{2}$ and Young's inequality we obtain

$$
\begin{aligned}
\int_{\Lambda_{r^{2}} \int_{B^{+}} \frac{\left|\tau_{h} u\right|^{2}}{|h|} \eta^{2} d x d t} & \leqslant \int_{\Lambda_{r^{2}}} \int_{B_{\varrho}^{+}} L\left(1+|D u|_{h}\right)\left(\left|D \tau_{h} u\right| \eta^{2}+\left|\tau_{h} u\right|\left|D \eta^{2}\right|\right)+\left|G_{h}\right|\left|\tau_{h} u\right| \eta^{2} d x d t \\
& \leqslant \frac{1}{2} \int_{\Lambda_{r^{2}}} \int_{B_{\varrho}^{+}} \frac{\left|\tau_{h} u\right|^{2}}{|h|} \eta^{2} d x d t+c \int_{\Lambda_{r^{2}}} \int_{B_{\varrho}^{+}} 1+\left|\tau_{h} D u\right|^{2}+|D u|_{h}^{2}+\varrho^{2}\left|G_{h}\right|^{2} d x d t
\end{aligned}
$$

where $c=c(L)$. Now, we can absorb the first term of the right-hand side into the left. Using $\eta \equiv 1$ on $B_{r}$ and $0<|h| \leqslant(\varrho-r)^{2} \leqslant \varrho^{2}-r^{2}$ the preceding inequality leads us to

$$
\int_{Q_{r}^{+}} \frac{\left|\tau_{h} u\right|^{2}}{|h|} d z \leqslant c \int_{-r^{2}}^{r^{2}} \int_{B^{+}}\left(1+\left|\tau_{h} D u\right|^{2}+|D u|_{h}^{2}+\varrho^{2}\left|G_{h}\right|^{2}\right) d x d t \leqslant c \int_{Q_{\varrho}^{+}}\left(1+|D u|^{2}+\varrho^{2}|G|^{2}\right) d z,
$$

where $c$ depends on $L$ only. This proves the desired estimate.

Lemma 4.5. Let $u$ be a weak solution of (4.1) on $Q_{R}^{+}$according to Definition 4.1. Then, for all $0<r<\varrho \leqslant R$ and $0<|h| \leqslant\left(R^{2}-\varrho^{2}\right) / 2$ and $\varepsilon \in(0,1]$ there holds

$$
\begin{aligned}
& \sup _{t \in \Lambda_{r^{2}}} \int_{B_{r}^{+}}\left|\tau_{h} u(\cdot, t)\right|^{2} d x+\int_{Q_{r}^{+}}\left|\tau_{h} D u\right|^{2} d z \\
& \quad \leqslant \varepsilon \int_{Q_{\varrho}^{+}} \frac{\left|\tau_{-h} \tau_{h} u\right|^{2}}{|h|} d z+\frac{c}{(\varrho-r)^{2}} \int_{Q_{Q}^{+}}\left|\tau_{h} u\right|^{2} d z+c|h|\left[|h|^{\vartheta} \gamma^{2} \int_{Q_{\varrho}^{+}}\left(1+|D u|^{2}\right) d z+\frac{1}{\varepsilon} \int_{Q_{Q}^{+}}|\mathcal{G}|^{2} d z\right],
\end{aligned}
$$

where $c=c(L / \nu)$ and $\mathcal{G}(x, t):=|G(x, t)|+\left|\tau_{h} G(x, t)\right|$.

Proof. Taking the difference of the Steklov-formulation (4.3) of the parabolic system (with $h$ replaced by $\lambda$ ) at level $s+h$ and $s$ we obtain for a.e. $s \in \Lambda_{R^{2}}$ that

$$
\int_{B_{R}^{+}} \partial_{t}\left(\tau_{h} u_{\lambda}\right)(\cdot, s) \cdot \varphi+\left\langle\tau_{h}[a(\cdot, s, D u)]_{\lambda}, D \varphi\right\rangle d x=\int_{B_{R}^{+}}\left\langle\tau_{h} G_{\lambda}(\cdot, s), \varphi\right\rangle d x .
$$


In this equation we choose the test-function $\varphi(x, t)=\eta^{2}(x) \zeta(t) \tau_{h}\left[u_{\lambda}\right](x, t)$, where $\eta \in C_{0}^{\infty}\left(B_{\varrho}\right), \zeta \in C_{0}^{1}\left(\Lambda_{\varrho^{2}}\right)$ are cut-off functions with $0 \leqslant \eta \leqslant 1, \eta \equiv 1$ on $B_{r},|D \eta| \leqslant 2 /(\varrho-r), 0 \leqslant \zeta \leqslant 1, \zeta \equiv 1$ on $\Lambda_{r^{2}}$ and $\left|\zeta^{\prime}\right| \leqslant 2 /(\varrho-r)^{2}$, and then integrate with respect to time (the variable will be denoted by $s$ ) over $\left(-\varrho^{2}, t\right)$, with $t \in \Lambda_{\varrho^{2}}$. Proceeding in this way and noting that

$$
\begin{aligned}
\int_{-\varrho^{2} B_{\varrho}^{+}}^{t} \int_{t} \partial_{t}\left(\tau_{h} u_{\lambda}\right) \cdot\left(\tau_{h} u_{\lambda}\right) \eta^{2} \zeta d x d s & =\frac{1}{2} \int_{-\varrho^{2} B_{\varrho}^{+}}^{t} \partial_{t}\left|\tau_{h} u_{\lambda}\right|^{2} \zeta \eta^{2} d x d s \\
& =\frac{1}{2} \int_{B_{\varrho}^{+}}\left|\tau_{h} u_{\lambda}(\cdot, t)\right|^{2} \eta^{2} \zeta(t) d x-\frac{1}{2} \int_{-\varrho^{2}}^{t} \int_{B_{\varrho}^{+}}\left|\tau_{h} u_{\lambda}\right|^{2} \eta^{2} \zeta^{\prime} d x d s
\end{aligned}
$$

we infer that

$$
\begin{aligned}
& \frac{1}{2} \int_{B_{\varrho}^{+}}\left|\tau_{h} u_{\lambda}(\cdot, t)\right|^{2} \eta^{2} \zeta(t) d x+\int_{-\varrho^{2} B_{\varrho}^{+}}^{t}\left\langle\tau_{h}[a(\cdot, D u)]_{\lambda}, D\left(\eta^{2} \tau_{h} u_{\lambda}\right)\right| \zeta d x d s \\
& \quad=\int_{-\varrho^{2}}^{t} \int_{B_{\varrho}^{+}} \frac{1}{2}\left|\tau_{h} u_{\lambda}\right|^{2} \eta^{2} \zeta^{\prime}-\left\langle\tau_{h} G_{\lambda}, \tau_{h} u\right\rangle \eta^{2} \zeta d x d s .
\end{aligned}
$$

Passing to the limit $\lambda \downarrow 0$ and rearranging terms we find that for a.e. $t \in \Lambda_{\varrho^{2}}$ there holds

$$
\begin{aligned}
& \frac{1}{2} \int_{B_{\varrho}^{+}}\left|\tau_{h} u(\cdot, t)\right|^{2} \eta^{2} \zeta(t) d x+\int_{-\varrho^{2} B_{\varrho}^{+}}^{t}\left\langle\mathcal{A}(h)+\mathcal{B}(h), \tau_{h} D u\right| \eta^{2} \zeta d x d s \\
& \quad=-2 \int_{-\varrho^{2}}^{t} \int_{B_{\varrho}^{+}}\left\langle\mathcal{A}(h)+\mathcal{B}(h),\left.D \eta \otimes \tau_{h} u\left|\eta \zeta d x d s+\int_{-\varrho^{2}}^{t} \int_{B_{\varrho}^{+}} \frac{1}{2}\right| \tau_{h} u\right|^{2} \eta^{2} \zeta^{\prime}-\left\langle\tau_{h} G, \tau_{h} u\right\rangle \eta^{2} \zeta d x d s,\right.
\end{aligned}
$$

where we have used the abbreviation

$$
\tau_{h}[a(\cdot, \cdot, D u(\cdot, \cdot))](x, s)=\mathcal{A}(h)(x, s)+\mathcal{B}(h)(x, s)
$$

with

$$
\begin{aligned}
\mathcal{A}(h)(x, s) & :=a(x, s+h, D u(x, s+h))-a(x, s+h, D u(x, s)) \\
& =\int_{0}^{1} \partial_{w} a\left(x, s+h, D u(x, s)+\sigma \tau_{h}[D u](x, s)\right) d \sigma \tau_{h}[D u](x, s)
\end{aligned}
$$

and $\mathcal{B}(h)(x, s):=a(x, s+h, D u(x, s))-a(x, s, D u(x, s))$. From the ellipticity condition (4.2) 2 we infer for the integral involving $\mathcal{A}(h)$ on the left-hand side of (4.7) that

$$
\int_{-\varrho^{2}}^{t} \int_{B_{\varrho}^{+}}\left\langle\mathcal{A}(h),\left.\tau_{h} D u\left|\eta^{2} \zeta d x d s \geqslant v \int_{-\varrho^{2}}^{t} \int_{B_{\varrho}^{+}}\right| \tau_{h}[D u]\right|^{2} \eta^{2} \zeta d x d s .\right.
$$

On the other hand, using the upper bound (4.2) 1 and Young's inequality with $\mu_{1}>0$ (to be chosen later) we obtain for the integral involving $\mathcal{A}(h)$ on the right-hand side of (4.7) that 


$$
\begin{aligned}
2 \mid \int_{-\varrho^{2}} \int_{B_{\varrho}^{+}}\left\langle\mathcal{A}(h), D \eta \otimes \tau_{h} u|\eta \zeta d x d s|\right. & \leqslant 2 L \int_{-\varrho^{2}}^{t} \int_{B^{+}}\left|\tau_{h}[D u]\right|\left|D \eta \| \tau_{h} u\right| \eta \zeta d x d s \\
& \leqslant \mu_{1} \int_{-\varrho^{2}}^{t} \int_{B_{\varrho}^{+}}\left|\tau_{h}[D u]\right|^{2} \eta^{2} \zeta d x d s+\frac{L^{2}}{\mu_{1}} \int_{Q_{\varrho}^{+}}|D \eta|^{2}\left|\tau_{h} u\right|^{2} d z .
\end{aligned}
$$

To estimate the integrals involving $\mathcal{B}(h)$ in (4.7) we exploit the Lipschitz continuity of the vector field $a(x, t, w)$ with respect to $t$ from $(4.2)_{4}$ in the form

$$
|\mathcal{B}(h)(x, s)| \leqslant L \gamma|h|^{\frac{1+\vartheta}{2}}(1+|D u(x, s)|) .
$$

Using Young's inequality again we find

$$
\mid \int_{-\varrho^{2} B_{\varrho}^{+}}^{t}\left\langle\mathcal{B}(h), D \tau_{h} u\left|\eta^{2} \zeta d x d s\right| \leqslant \mu_{1} \int_{-\varrho^{2} B_{\varrho}^{+}}^{t}\left|\tau_{h}[D u]\right|^{2} \eta^{2} \zeta d x d s+c_{\mu_{1}} \int_{Q_{\varrho}^{+}}(1+|D u|)^{2} d z\right.
$$

and similarly

$$
2 \mid \int_{-\varrho^{2}}^{t} \int_{B^{+}}\left\langle\mathcal{B}(h), D \eta \otimes \tau_{h} u|\eta \zeta d x d s| \leqslant \mu_{1} \int_{Q_{\varrho}^{+}}|D \eta|^{2}\left|\tau_{h} u\right|^{2} d z+c_{\mu_{1}} \int_{Q_{\varrho}^{+}}(1+|D u|)^{2} d z,\right.
$$

where $c_{\mu_{1}}=L^{2} \gamma^{2}|h|^{1+\vartheta} \mu_{1}^{-1}$. Finally, we estimate the term in (4.7) involving the right side $G$. In the case $(\varrho-r)^{2} \leqslant|h|$ we use Young's inequality to obtain

$$
\int_{-\varrho^{2}}^{t} \int_{B_{\varrho}^{+}}\left\langle\tau_{h} G, \tau_{h} u\right\rangle \eta^{2} \zeta d x d s \leqslant|h| \int_{Q_{\varrho}^{+}}\left|\tau_{h} G\right|^{2} d z+\frac{1}{|h|} \int_{Q_{\varrho}^{+}}\left|\tau_{h} u\right|^{2} d z \leqslant|h| \int_{Q_{\varrho}^{+}}\left|\tau_{h} G\right|^{2} d z+\frac{1}{(\varrho-r)^{2}} \int_{Q_{\varrho}^{+}}\left|\tau_{h} u\right|^{2} d z,
$$

while in the case $(\varrho-r)^{2}>|h|$ integration by parts for finite differences yields

$$
\begin{aligned}
\int_{-\varrho^{2}}^{t} \int_{B^{+}}\left\langle\tau_{h} G, \zeta \tau_{h} u\right\rangle \eta^{2} d x d s= & \int_{-\varrho^{2}}^{t} \int_{B_{\varrho}^{+}}\left\langle G, \tau_{-h}\left(\zeta \tau_{h} u\right)\right| \eta^{2} d x d s \\
& +\int_{t}^{t+h} \int_{B_{\varrho}^{+}}\left\langle G(\cdot, s), \zeta(s-h) \tau_{h} u(\cdot, s-h)\right| \eta^{2} d x d s \\
& -\int_{-\varrho^{2}}^{-\varrho^{2}+h}\left\langle G(\cdot, s), \zeta(s-h) \tau_{h} u(\cdot, s-h)\right| \eta^{2} d x d s \\
= & I+I I+I I I,
\end{aligned}
$$

with the obvious meaning of $I-I I I$. Note that the previous formula holds for positive as well as for negative parameters $h$; but for $h>0$ we already have $I I I=0$ since spt $\zeta \subset \Lambda_{Q^{2}}$. To estimate $I$ we use the identity

$$
\tau_{-h}\left(\zeta \tau_{h} u\right)(s)=\zeta(s-h) \tau_{-h} \tau_{h} u(s)+\tau_{-h} \zeta(s) \tau_{h} u(s)
$$

as well as $\zeta \leqslant 1$ and $\left|\tau_{-h} \zeta\right| \leqslant 2|h|(\varrho-r)^{-2}, t \leqslant \varrho^{2}$ and Young's inequality with $\varepsilon \in(0,1]$ to deduce 


$$
\begin{aligned}
I & =\int_{-\varrho^{2}}^{t} \int_{B_{\varrho}^{+}}\left\langle G(\cdot, s), \zeta(s-h) \tau_{-h} \tau_{h} u(\cdot, s)+\tau_{-h} \zeta(s) \tau_{h} u(\cdot, s)\right| \eta^{2} d x d s \\
& \leqslant \int_{Q_{\varrho}^{+}}|G|\left|\tau_{-h} \tau_{h} u\right| d z+\frac{2|h|}{(\varrho-r)^{2}} \int_{Q_{\varrho}^{+}}|G|\left|\tau_{h} u\right| d z \\
& \leqslant \varepsilon \int_{Q_{\varrho}^{+}} \frac{\left|\tau_{-h} \tau_{h} u\right|^{2}}{|h|} d z+\frac{2|h|}{\varepsilon} \int_{Q_{\varrho}^{+}}|G|^{2} d z+\frac{\varepsilon|h|}{(\varrho-r)^{4}} \int_{Q_{\varrho}^{+}}\left|\tau_{h} u\right|^{2} d z \\
& \leqslant \varepsilon \int_{Q_{\varrho}^{+}} \frac{\left|\tau_{-h} \tau_{h} u\right|^{2}}{|h|} d z+\frac{2|h|}{\varepsilon} \int_{Q_{\varrho}^{+}}|G|^{2} d z+\frac{\varepsilon}{(\varrho-r)^{2}} \int_{Q_{\varrho}^{+}}\left|\tau_{h} u\right|^{2} d z,
\end{aligned}
$$

where in the last line we have taken into account that $|h|<(\varrho-r)^{2}$. To estimate $I I$ we in turn perform a transformation, apply Young's inequality (with $\mu_{2}>0$ to be chosen later; note also that $\operatorname{spt} \zeta \subset \Lambda_{\varrho^{2}}$ ) and estimate $|G(\cdot, s+h)|^{2}$ by $2|G(\cdot, s)|^{2}+2\left|\tau_{h} G(\cdot, s)\right|^{2}$. This leads us in the case $h>0$ to

$$
\begin{aligned}
I I & =\int_{t-h}^{t} \int_{B_{\varrho}^{+}}\left\langle G(\cdot, s+h), \zeta(s) \tau_{h} u(\cdot, s)\right| \eta^{2} d x d s \\
& \leqslant \frac{|h|}{2 \mu_{2}} \int_{t-h}^{t} \int_{B_{\varrho}^{+}}|G(\cdot, s+h)|^{2} \eta^{2} \zeta(s) d x d s+\frac{\mu_{2}}{2|h|} \int_{t-h}^{t} \int_{B_{\varrho}^{+}}\left|\tau_{h} u\right|^{2} \eta^{2} \zeta d x d s \\
& =\frac{|h|}{\mu_{2}} \int_{Q_{\varrho}^{+}}|G|^{2}+\left|\tau_{h} G\right|^{2} d z+\frac{\mu_{2}}{2|h|} \int_{(t-h, t) \cap \Lambda_{\varrho^{2}}} \int_{B_{\varrho}^{+}}\left|\tau_{h} u\right|^{2} \eta^{2} \zeta d x d s \\
& \leqslant \frac{|h|}{\mu_{2}} \int_{Q_{\varrho}^{+}}|G|^{2}+\left|\tau_{h} G\right|^{2} d z+\mu_{2} \sup _{s \in \Lambda_{\varrho^{2}}} \int_{B_{\varrho}^{+}}\left|\tau_{h} u(\cdot, s)\right|^{2} \eta^{2} \zeta(s) d x .
\end{aligned}
$$

In the case $h<0$ we obtain completely the same estimate when replacing the interval $(t-h, t)$ by $(t, t-h)$. In completely the same way we achieve the estimate for $I I I$, which reads as follows:

$$
I I I \leqslant \frac{|h|}{\mu_{2}} \int_{Q_{\varrho}^{+}}|G|^{2}+\left|\tau_{h} G\right|^{2} d z+\mu_{2} \sup _{s \in \Lambda_{Q^{2}}} \int_{B_{\varrho}^{+}}\left|\tau_{h} u(\cdot, s)\right|^{2} \eta^{2} \zeta(s) d x .
$$

Combining the preceding estimates for $I-I I I$ with (4.9) we deduce a bound for the integral involving the inhomogeneity also in the second case $|h|<(\varrho-r)^{2}$. Subsequently, joining the preceding estimates for the terms appearing in (4.7) and choosing $\mu_{1}$ as usual small enough (note that we can also switch to smaller values of $\varepsilon$ and $\mu_{2}$ if necessary) we finally arrive at

$$
\begin{aligned}
& \int_{B_{\varrho}^{+}}\left|\tau_{h} u(\cdot, t)\right|^{2} \eta^{2} \zeta(t) d x+\int_{-\varrho^{2}}^{t} \int_{B_{\varrho}^{+}}\left|\tau_{h} D u\right|^{2} \eta^{2} \zeta d x d s \\
& \leqslant 2 \mu_{2} \sup _{s \in \Lambda_{Q^{2}}} \int_{B_{\varrho}^{+}}\left|\tau_{h} u(\cdot, s)\right|^{2} \eta^{2} \zeta(s) d x+\varepsilon \int_{Q_{\varrho}^{+}} \frac{\left|\tau_{-h} \tau_{h} u\right|^{2}}{|h|} d z+\frac{c}{(\varrho-r)^{2}} \int_{Q_{\varrho}^{+}}\left|\tau_{h} u\right|^{2} d z \\
& \quad+c|h|^{1+\vartheta} \gamma^{2} \int_{Q_{\varrho}^{+}}\left(1+|D u|^{2}\right) d z+|h|\left(\varepsilon^{-1}+\mu_{2}^{-1}\right) \int_{Q_{\varrho}^{+}}|G|^{2}+\left|\tau_{h} G\right|^{2} d z .
\end{aligned}
$$




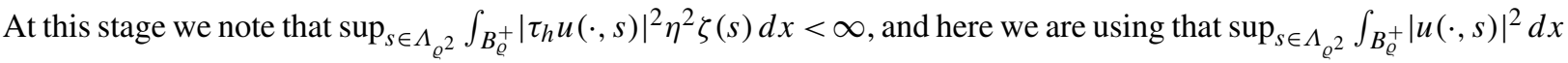
is finite. Indeed, in the parabolic setting this expression appears on the left-hand side of the Caccioppoli inequality (see for instance the proof of Lemma 3.1). Since (4.10) holds for a.e. $t \in \Lambda_{\varrho^{2}}$ we can take the supremum over $t \in \Lambda_{\varrho^{2}}$ in the first term on the left-hand side and let $t \uparrow \varrho^{2}$ in the second one. Choosing $\mu_{2}$ small enough, absorbing the sup-term of the right-hand side on the left and recalling that $\eta^{2} \zeta \equiv 1$ on $Q_{r}^{+}$the preceding estimate yields the desired estimate for positive finite differences, i.e. for $\tau_{h} u$, respectively $\tau_{h} D u$. This proves the assertion of the lemma with a constant $c=c(L / v)$.

Corollary 4.6. Let $u$ be a weak solution of (4.1) on $Q_{R}^{+}$according to Definition 4.1. Then, for all $0<r<\varrho \leqslant R$ and $0<|h| \leqslant\left(R^{2}-\varrho^{2}\right) / 2$ and $\varepsilon>0$ there holds, with $c=c(L / v)$

$$
\begin{aligned}
& \sup _{t \in \Lambda_{r^{2}}} \int_{B_{r}^{+}}\left|\tau_{h} u(\cdot, t)\right|^{2} d x+\int_{Q_{r}^{+}}\left|\tau_{h} D u\right|^{2} d z \\
& \quad \leqslant c\left(\frac{1}{(\varrho-r)^{2}}+\frac{1}{\varepsilon}\right) \int_{Q_{\varrho}^{+}}\left|\tau_{h} u\right|^{2} d z+c|h|^{1+\vartheta} \gamma^{2} \int_{Q_{\varrho}^{+}}\left(1+|D u|^{2}\right) d z+\varepsilon \int_{Q_{\varrho}^{+}}\left|\tau_{h} G\right|^{2} d z .
\end{aligned}
$$

Proof. We proceed completely similar to the proof of Lemma 4.5 except for the term in (4.7) involving the right side $G$. Here we now apply Young's inequality to obtain

$$
\int_{-\varrho^{2} B_{\varrho}^{+}}^{t}\left\langle\tau_{h} G, \tau_{h} u\right\rangle \eta^{2} \zeta d x d s \leqslant \varepsilon \int_{Q_{\varrho}^{+}}\left|\tau_{h} G\right|^{2} d z+\frac{1}{\varepsilon} \int_{Q_{\varrho}^{+}}\left|\tau_{h} u\right|^{2} d z .
$$

Inserting this in (4.7) and replacing $\varepsilon$ by a smaller value if necessary, this leads us to the desired estimate.

Lemma 4.7. Let $u$ be a weak solution of (4.1) on $Q_{R}^{+}$according to Definition 4.1. Then, for all $z_{0}=\left(x_{0}, t_{0}\right) \in Q_{R}^{+} \cup \Gamma_{R}$ and $0<r<\varrho \leqslant R$ and $0<|h| \leqslant \varrho^{2}$ such that $Q_{2 \varrho}^{+}\left(z_{0}\right) \subset Q_{R}^{+}$there holds

$$
\begin{aligned}
\int_{Q_{r}^{+}\left(z_{0}\right)} \frac{\left|\tau_{-h} \tau_{h} u\right|^{2}}{|h|} d z \leqslant & c\left(1+\frac{|h|}{(\varrho-r)^{2}}\right) \int_{Q_{\varrho}^{+}\left(z_{0}\right)}\left|\tau_{h} D u\right|^{2}+\left|\tau_{-h} D u\right|^{2} d z \\
& +c|h|^{1+\vartheta} \gamma^{2}\left(1+\frac{|h|}{(\varrho-r)^{2}}\right) \int_{Q_{\varrho+\sqrt{|h|}}^{+}\left(z_{0}\right)}\left(1+|D u|^{2}\right) d z \\
& +c|h| \int_{Q_{\varrho}^{+}\left(z_{0}\right)}\left|\tau_{h} G\right|^{2}+\left|\tau_{-h} G\right|^{2} d z,
\end{aligned}
$$

where $c=c(L)$. (Note that $Q_{r}^{+}\left(z_{0}\right), Q_{\varrho}^{+}\left(z_{0}\right)$ are not necessarily half-cylinders.)

Proof. For convenience in notation we omit the reference to the point $z_{0}$ in the following and without loss of generality we assume that $t_{0}=0$. Moreover, since $\tau_{-h} \tau_{h} u=\tau_{h} \tau_{-h} u$ we can assume without loss of generality that $h>0$. We start with the Steklov-formulation (4.3) of the system and take its difference at level $t-h$ and $t$. Then, for a.e. $t \in \Lambda_{R^{2}}$ we have

$$
\int_{B_{R}^{+}} \tau_{-h} \partial_{t} u_{h}(\cdot, t) \cdot \varphi+\left\langle\tau_{-h}[a(\cdot, t, D u)]_{h}, D \varphi\right\rangle d x=\int_{B_{R}^{+}}\left\langle\tau_{-h} G_{h}(\cdot, t), \varphi\right\rangle d x .
$$

In this equation we choose the test-function $\varphi(x, t)=\eta^{2}(x) \tau_{-h} \tau_{h} u(x, t)$, where $\eta \in C_{0}^{\infty}\left(B_{\varrho}\right)$ is a cut-off function with $0 \leqslant \eta \leqslant 1, \eta \equiv 1$ on $B_{r},|D \eta| \leqslant 2 /(\varrho-r)$. Integrating the resulting equation with respect to $t$ over $\Lambda_{\varrho^{2}}$ and using also $\partial_{t} u_{h}=\tau_{h} u / h$ we obtain 


$$
\begin{aligned}
\int_{Q_{\varrho}^{+}} \frac{\left|\tau_{-h} \tau_{h} u\right|^{2}}{h} \eta^{2} d z & =\int_{Q_{\varrho}^{+}} \tau_{-h}\left(\partial_{t} u_{h}\right) \cdot \eta^{2} \tau_{-h} \tau_{h} u d z \\
& =\int_{Q_{\varrho}^{+}}\left\langle\tau_{-h} G_{h}, \eta^{2} \tau_{-h} \tau_{h} u\right\rangle+\left\langle\tau_{-h}[a(\cdot, D u)]_{h}, D\left(\eta^{2} \tau_{-h} \tau_{h} u\right)\right\rangle d z .
\end{aligned}
$$

With the notation introduced in (4.8) we decompose

$$
\tau_{-h}[a(\cdot, D u)]_{h}=\left[\tau_{-h} a(\cdot, D u)\right]_{h}=:[\mathcal{A}(-h)]_{h}+[\mathcal{B}(-h)]_{h} .
$$

Using this and Young's inequality we obtain from the second last equation for $\varepsilon_{1}, \varepsilon_{2}>0$ (to be chosen later) that

$$
\begin{aligned}
\int_{Q_{\varrho}^{+}} \frac{\left|\tau_{-h} \tau_{h} u\right|^{2}}{h} \eta^{2} d z \leqslant & \int_{Q_{\varrho}^{+}}\left|D\left(\tau_{-h} \tau_{h} u\right)\right|^{2} \eta^{2}+\varepsilon_{1}\left|D \eta^{2}\right|^{2}\left|\tau_{-h} \tau_{h} u\right|^{2}+\varepsilon_{2}\left|\tau_{-h} \tau_{h} u\right|^{2} \eta^{2} d z \\
& +\int_{Q_{\varrho}^{+}}\left(1+\varepsilon_{1}^{-1}\right)\left(\left|[\mathcal{A}(-h)]_{h}\right|^{2}+\left|[\mathcal{B}(-h)]_{h}\right|^{2}\right)+\varepsilon_{2}^{-1}\left|\tau_{-h} G_{h}\right|^{2} d z \\
= & : I_{1}+\varepsilon_{1} I_{2}+\varepsilon_{2} I_{3}+\left(1+\varepsilon_{1}^{-1}\right)\left(I_{4}+I_{5}\right)+\varepsilon_{2}^{-1} I_{6},
\end{aligned}
$$

with the obvious meaning of $I_{1}-I_{6}$. In turn we will estimate these terms. We start with the estimate for $I_{1}$. Using (2.8) we find

$$
I_{1} \leqslant 2 \int_{Q_{\varrho}^{+}}\left|\tau_{h} D u\right|^{2}+\left|\tau_{-h} D u\right|^{2} d z
$$

The estimate for $I_{2}$ is achieved by the use of $|D \eta| \leqslant 2 /(\varrho-r)$ as follows:

$$
I_{2} \leqslant \frac{8}{(\varrho-r)^{2}} \int_{Q_{\varrho}^{+}}\left|\tau_{-h} \tau_{h} u\right|^{2} \eta^{2} d z
$$

In order to get an estimate for $I_{4}$ we use in turn $(4.2)_{1}$ and the fact that $|h| \leqslant \varrho^{2}$ to find

$$
I_{4} \leqslant \int_{-\varrho^{2}}^{\varrho^{2}+h} \int_{B_{\varrho}^{+}}|\mathcal{A}(-h)|^{2} d x d t \leqslant L^{2} \int_{-\varrho^{2}}^{\varrho^{2}+h} \int_{B_{\varrho}^{+}}\left|\tau_{-h} D u\right|^{2} d x d t \leqslant L^{2} \int_{Q_{\varrho}^{+}}\left|\tau_{h} D u\right|^{2}+\left|\tau_{-h} D u\right|^{2} d z .
$$

For the estimate for $I_{5}$ we use $(4.2)_{3}$ and $\varrho^{2}+|h| \leqslant(\varrho+\sqrt{|h|})^{2}$ to infer

$$
I_{5} \leqslant \int_{-\varrho^{2}}^{\varrho^{2}} \int_{B_{\varrho}^{+}}|\mathcal{B}(-h)|^{2} d x d t \leqslant L^{2} \gamma^{2}|h|^{1+\vartheta} \int_{-\varrho^{2}}^{\varrho^{2}} \int_{B^{+}}(1+|D u|)^{2} d x d t \leqslant L^{2} \gamma^{2}|h|^{1+\vartheta} \int_{Q_{\varrho+\sqrt{|h|}}^{+}}(1+|D u|)^{2} d z .
$$

Finally, for the estimate for $I_{6}$ we decompose and enlarge the domain of integration in order to obtain

$$
I_{6} \leqslant \int_{-\varrho^{2}}^{\varrho^{2}} \int_{B_{\varrho}^{+}}\left|\tau_{-h} G\right|^{2} d x d t=\int_{-\varrho^{2}}^{\varrho^{2}} \int_{B^{+}}\left|\tau_{-h} G\right|^{2} d x d t+\int_{\varrho^{2}-h}^{\varrho^{2}} \int_{B_{\varrho}^{+}}\left|\tau_{h} G\right|^{2} d x d t \leqslant \int_{Q_{\varrho}^{+}}\left|\tau_{h} G\right|^{2}+\left|\tau_{-h} G\right|^{2} d z .
$$

Combining the previous estimates with (4.11), choosing $\varepsilon_{1}=\frac{(\varrho-r)^{2}}{8|h|}, \varepsilon_{2}=\frac{1}{4|h|}$ and absorbing terms we arrive at 


$$
\begin{aligned}
\int_{Q_{\varrho}^{+}} \frac{\left|\tau_{-h} \tau_{h} u\right|^{2}}{h} \eta^{2} d z \leqslant & c\left(1+\frac{|h|}{(\varrho-r)^{2}}\right) \int_{Q_{\varrho}^{+}}\left|\tau_{h} D u\right|^{2}+\left|\tau_{-h} D u\right|^{2} d z \\
& +c|h|^{1+\vartheta} \gamma^{2}\left(1+\frac{|h|}{(\varrho-r)^{2}}\right) \int_{Q_{\varrho+\sqrt{|h|}}^{+}}\left(1+|D u|^{2}\right) d z+c|h| \int_{Q_{\varrho}^{+}}\left|\tau_{h} G\right|^{2}+\left|\tau_{-h} G\right|^{2} d z,
\end{aligned}
$$

where $c=100 L^{2}$ for example. Together with $\eta \equiv 1$ on $B_{r}$ this yields the desired estimate.

Having arrived at this stage we can join the preceding results to achieve the weak differentiability of $u$ with respect to the time variable $t$. To be precise

Proposition 4.8. Let $u$ be a weak solution of (4.1) on $Q_{R}^{+}$according to Definition 4.1 and let $G \in \mathcal{N}^{0, \vartheta ; 2}\left(Q_{R}^{+} ; \mathbb{R}^{N}\right)$. Then, $\partial_{t} u \in L^{2}\left(Q_{r}^{+} ; \mathbb{R}^{N}\right)$ for any $0<r<R$ and for all $0<\varrho \leqslant R$ there holds

$$
\int_{Q_{\varrho / 2}^{+}}\left|\partial_{t} u\right|^{2} d z \leqslant c\left[\left(\gamma^{2}+\varrho^{-2}\right) \int_{Q_{\varrho}^{+}}\left(1+|D u|^{2}\right) d z+\|G\|_{\mathcal{N}^{0, \vartheta ; 2}\left(Q_{\varrho}^{+}\right)}^{2}\right] \text {, }
$$

where $c=c(L / \nu, \vartheta)$ and $c \rightarrow \infty$ when $\vartheta \downarrow 0$.

Proof. We consider $0<|h| \leqslant(\varrho / 4)^{2}$ and $\varrho / 4 \leqslant r<s \leqslant \varrho / 2$. In the sequel we use the notions $\mathcal{Z}_{h}(s, r):=1+$ $|h|(s-r)^{-2}$ and $\mathcal{Z}_{h}(\varrho):=1+|h| \varrho^{-2}$. Applying in turn Lemma 4.7 (with radii $r$ and $(s+r) / 2$ ) and Lemma 4.5 (for $h$ and $-h$ and with radii $(s+r) / 2, s$ and $\left.\varepsilon=\left[2 c \mathcal{Z}_{h}(s, r)\right]^{-1}\right)$ and noting that $(s+r) / 2+\sqrt{|h|}<\varrho / 2+\varrho / 4<\varrho$ as well as $[(s+r) / 2]^{2}+|h|<(\varrho / 2)^{2}+(\varrho / 4)^{2}<\varrho^{2}$ and $|h|^{\vartheta} \leqslant 1$ we obtain

$$
\begin{aligned}
\int_{Q_{r}^{+}} \frac{\left|\tau_{-h} \tau_{h} u\right|^{2}}{|h|} d z \leqslant & c \mathcal{Z}_{h}(s, r) \int_{Q_{(s+r) / 2}^{+}}\left|\tau_{h} D u\right|^{2}+\left|\tau_{-h} D u\right|^{2} d z \\
& +c|h|\left[\gamma^{2} \mathcal{Z}_{h}(s, r) \int_{Q_{\varrho}^{+}}\left(1+|D u|^{2}\right) d z+\int_{Q_{\varrho}^{+}}|G|^{2} d z\right] \\
\leqslant & \frac{1}{2} \int_{Q_{s}^{+}} \frac{\left|\tau_{-h} \tau_{h} u\right|^{2}}{|h|} d z+c \frac{\mathcal{Z}_{h}(s, r)}{(s-r)^{2}} \int_{Q_{\varrho / 2}^{+}}\left|\tau_{h} u\right|^{2}+\left|\tau_{-h} u\right|^{2} d z \\
& +c|h| \mathcal{Z}_{h}(s, r)\left[\gamma^{2} \int_{Q_{\varrho}^{+}}\left(1+|D u|^{2}\right) d z+\int_{Q_{\varrho}^{+}}|G|^{2} d z\right],
\end{aligned}
$$

where $c=c(L / v)$. Applying the iteration lemma [7, Lemma 2.4] we can absorb the first integral on the right-hand side in the preceding estimate on the left to infer

$$
\begin{aligned}
\int_{Q_{\varrho / 4}^{+}} \frac{\left|\tau_{-h} \tau_{h} u\right|^{2}}{|h|} d z \leqslant & c \frac{\mathcal{Z}_{h}(\varrho)}{\varrho^{2}} \int_{Q_{\varrho / 2}^{+}}\left|\tau_{h} u\right|^{2}+\left|\tau_{-h} u\right|^{2} d z \\
& +c|h| \mathcal{Z}_{h}(\varrho)\left[\gamma^{2} \int_{Q_{\varrho}^{+}}\left(1+|D u|^{2}\right) d z+\int_{Q_{\varrho}^{+}}|G|^{2} d z\right] .
\end{aligned}
$$

Since $|h| \leqslant(\varrho / 4)^{2}$ we have $\mathcal{Z}_{h}(\varrho) \leqslant 2$. On the other hand, applying Lemma 4.4 with $h$ and $-h$ we see that

$$
\int_{Q_{\varrho / 2}^{+}}\left|\tau_{h} u\right|^{2}+\left|\tau_{-h} u\right|^{2} d z \leqslant c|h| \int_{Q_{\varrho}^{+}}\left(1+|D u|^{2}+\varrho^{2}|G|^{2}\right) d z .
$$


Inserting this in the second last estimate we arrive at

$$
\int_{Q_{\varrho / 4}^{+}}\left|\tau_{-h} \tau_{h} u\right|^{2} d z \leqslant c h^{2} M^{2}
$$

where we have set

$$
M^{2}:=\left(\gamma^{2}+\varrho^{-2}\right) \int_{Q_{\varrho}^{+}}\left(1+|D u|^{2}\right) d z+\int_{Q_{\varrho}^{+}}|G|^{2} d z .
$$

Since $\tau_{-h} \tau_{h} u(t)=\tau_{h} \tau_{h} u(t-h)$ this implies

$$
\int_{Q_{\varrho / 8}^{+}}\left|\tau_{h} \tau_{h} u\right|^{2} d z \leqslant \int_{Q_{\varrho / 4}^{+}}\left|\tau_{-h} \tau_{h} u\right|^{2} d z \leqslant c h^{2} M^{2},
$$

for any choice of $0<|h| \leqslant(\varrho / 8)^{2}$, and $c=c(L / \nu)$. At this stage we are in a position to apply Lemma 2.4 with $\left(1,1-\vartheta / 4, c M,(\varrho / 8)^{2}, 2\right)$ instead of $\left(\alpha, \beta, M, h_{0}, \sigma\right)$ yielding

$$
\int_{Q_{\varrho / 8}^{+}}\left|\tau_{h} u\right|^{2} d z \leqslant c|h|^{2-\frac{\vartheta}{2}}\left[\varrho^{\vartheta} M^{2}+\varrho^{\vartheta-4} \int_{Q_{\varrho}^{+}}|u|^{2} d z\right] \leqslant c|h|^{2-\frac{\vartheta}{2}} \varrho^{\vartheta} M^{2},
$$

for all $0<|h| \leqslant(\varrho / 16)^{2}$. Here we have also used the Poincaré inequality from Lemma 2.2 to estimate $\int_{Q_{\varrho}^{+}}|u|^{2} d z$. Moreover, the constant $c$ depends on $L / v$ and $\vartheta$ and we have $c \rightarrow \infty$ when $\vartheta \downarrow 0$. Now, we want to improve this estimate in the sense that we attain $h^{2}$ instead of $|h|^{2-\frac{\vartheta}{2}}$ on the right-hand side. For this we apply in turn Lemma 4.7 (with radii $\varrho / 32, \varrho / 16$ ) and Corollary 4.6 (for $h$ and $-h$ and with radii $\varrho / 16, \varrho / 8$ and $\varepsilon=|h|^{1-\vartheta} \varrho^{2 \vartheta}$ ). Taking also into account that $\varrho^{-2} \leqslant|h|^{\vartheta-1} \varrho^{-2 \vartheta}$ and $|h|^{-\vartheta} \varrho^{2 \vartheta} \geqslant 1$ this yields

$$
\begin{aligned}
\int_{Q_{\varrho / 32}^{+}} \frac{\left|\tau_{-h} \tau_{h} u\right|^{2}}{|h|} d z \leqslant & c \int_{Q_{\varrho / 16}^{+}}\left|\tau_{h} D u\right|^{2}+\left|\tau_{-h} D u\right|^{2} d z \\
& +c|h|\left[|h|^{\vartheta} \gamma^{2} \int_{Q_{\varrho / 8}^{+}}\left(1+|D u|^{2}\right) d z+\int_{Q_{\varrho / 16}^{+}}\left|\tau_{h} G\right|^{2}+\left|\tau_{-h} G\right|^{2} d z\right] \\
\leqslant & c|h|^{\vartheta-1} \varrho^{-2 \vartheta} \int_{Q_{\varrho / 8}^{+}\left|\tau_{h} u\right|^{2}+\left|\tau_{-h} u\right|^{2} d z} \\
& +c|h|\left[|h|^{\vartheta} \gamma^{2} \int_{Q_{\varrho / 8}^{+}}\left(1+|D u|^{2}\right) d z+|h|^{-\vartheta} \varrho_{\varrho^{2 \vartheta}}^{\int}\left|\tau_{h} G\right|^{2}+\left|\tau_{-h} G\right|^{2} d z\right] .
\end{aligned}
$$

Now, recalling the definition of $M$, using (4.14) for $h$ and $-h$ as well as the assumption $G \in \mathcal{N}^{0, \vartheta ; 2}\left(Q_{R}^{+} ; \mathbb{R}^{N}\right)$ leads us to

$$
\begin{aligned}
\int_{Q_{\varrho / 32}^{+}} \frac{\left|\tau_{-h} \tau_{h} u\right|^{2}}{|h|} d z & \leqslant c|h|\left[\left(|h|^{\frac{\vartheta}{2}} \varrho^{-\vartheta}+|h|^{\vartheta}\right) M^{2}+c|h|^{\vartheta} \varrho^{2 \vartheta}\|G\|_{\mathcal{N}^{0, \vartheta ; 2}\left(Q_{\varrho}^{+}\right)}^{2}\right] \\
& \leqslant c|h|^{1+\frac{\vartheta}{2}} \varrho^{-\vartheta}\left[M^{2}+\|G\|_{\mathcal{N}^{0, \vartheta ; 2}\left(Q_{\varrho}^{+}\right)}^{2}\right],
\end{aligned}
$$

for any $0<|h| \leqslant(\varrho / 16)^{2}$ and with a constant $c=c(L / \nu, \vartheta)$. Using once again the fact that $\tau_{-h} \tau_{h} u(t)=\tau_{h} \tau_{h} u(t-h)$ we therefore arrive at 


$$
\int_{Q_{\varrho / 64}^{+}}\left|\tau_{h} \tau_{h} u\right|^{2} d z \leqslant \int_{Q_{\varrho / 32}^{+}}\left|\tau_{-h} \tau_{h} u\right|^{2} d z \leqslant c|h|^{2+\frac{\vartheta}{2}} \varrho^{-\vartheta}\left[M^{2}+\|G\|_{\mathcal{N}^{0, \vartheta ; 2}\left(Q_{\varrho}^{+}\right)}^{2}\right],
$$

valid for any $0<|h| \leqslant(\varrho / 64)^{2}$. At this stage we can apply Lemma 2.4 a second time, but now with the choice $\left(1+\frac{\vartheta}{4}, 1, c\left(\varrho^{-\vartheta} M^{2}+\|G\|_{\mathcal{N}^{0, \vartheta ; 2}\left(Q_{\varrho}^{+}\right)}^{2}\right),(\varrho / 128)^{2}\right)$ for $\left(\alpha, \beta, M, h_{0}\right)$. This yields

$$
\begin{aligned}
\int_{Q_{\varrho / 64}^{+}}\left|\tau_{h} u\right|^{2} d z & \leqslant c h^{2}\left[M^{2}+\|G\|_{\mathcal{N}^{0, \vartheta ; 2}\left(Q_{\varrho}^{+}\right)}^{2}+\varrho^{-4} \int_{Q_{\varrho}^{+}}|u|^{2} d z\right] \\
& \leqslant c h^{2}\left[M^{2}+\|G\|_{\mathcal{N}^{0, \vartheta ; 2}\left(Q_{\varrho}^{+}\right)}^{2}\right],
\end{aligned}
$$

for any $0<|h| \leqslant(\varrho / 128)^{2}$ and where $c=c(L / v)$. Note that we have used the Poincaré inequality from Lemma 2.2 in the last line. The preceding estimate implies $\partial_{t} u \in L^{2}\left(Q_{R / 64}^{+} ; \mathbb{R}^{N}\right)$. Moreover, there holds

$$
\int_{Q_{\varrho / 64}^{+}}\left|\partial_{t} u\right|^{2} d z \leqslant c\left[M^{2}+\|G\|_{\mathcal{N}^{0, \vartheta ; 2}\left(Q_{\varrho}^{+}\right)}^{2}\right] .
$$

Finally, we note that by a different choice of the radii we can also attain $Q_{\varrho / 2}^{+}$instead of $Q_{\varrho / 64}^{+}$as domain of integration in the integral on the left-hand side. Recalling the definition of $M$ then proves the desired estimate. Further, let us note that again by a different choice of the involved radii and cylinder we also find that $\partial_{t} u \in L^{2}\left(Q_{r}^{+}, \mathbb{R}^{N}\right)$ for any $0<r<R$. This finishes the proof of the lemma.

Later, in the proof of the dimension reduction we will need an estimate for finite differences in time of the gradients of solutions of certain comparison systems. The following corollary will provide this estimate. It is an immediate consequence of the results we have obtained so far.

Corollary 4.9. Let $u$ be a weak solution of (4.1) on $Q_{R}^{+}$according to Definition 4.1 and let $G \in \mathcal{N}^{0, \vartheta ; 2}\left(Q_{R}^{+}\right)$. Then for all $0<\varrho \leqslant R$ and $0<|h| \leqslant(\varrho / 4)^{2}$ there holds

$$
\int_{Q_{\varrho / 2}^{+}}\left|\tau_{h} D u\right|^{2} d z \leqslant c(L / v, \vartheta)|h|\left[\left(\gamma^{2}+\varrho^{-2}\right) \int_{Q_{\varrho}^{+}}\left(1+|D u|^{2}\right) d z+\|G\|_{\mathcal{N}^{0, \vartheta ; 2}\left(Q_{\varrho}^{+}\right)}^{2}\right],
$$

where $c(L / \nu, \vartheta) \rightarrow \infty$ when $\vartheta \downarrow 0$.

Proof. Applying Lemma 4.5 with the choice $(\varrho / 2,3 \varrho / 4,1)$ for $(r, \varrho, \varepsilon)$ and noting that $\left|\tau_{-h} \tau_{h} u(t)\right| \leqslant\left|\tau_{h} u(t)\right|+$ $\left|\tau_{h} u(t-h)\right|$ we infer for $0<|h| \leqslant(\varrho / 4)^{2}\left(\right.$ then $(3 \varrho / 4)^{2}+|h| \leqslant \varrho^{2}$ and $\left.(3 \varrho / 4-\varrho / 2)^{-2}=(\varrho / 4)^{-2} \leqslant|h|^{-1}\right)$ that

$$
\int_{Q_{\varrho / 2}^{+}}\left|\tau_{h} D u\right|^{2} d z \leqslant c|h|^{-1} \int_{Q_{\varrho}^{+}}\left|\tau_{h} u\right|^{2} d z+c|h| \int_{Q_{\varrho}^{+}} \gamma^{2}\left(1+|D u|^{2}\right)+|G|^{2} d z .
$$

In the preceding inequality we are going to use the fact that $u$ is weakly differentiable with respect to time by Proposition 4.8 and the $L^{2}$-estimate for $\partial_{t} u$. This leads us to

$$
\int_{Q_{\varrho / 2}^{+}}\left|\tau_{h} D u\right|^{2} d z \leqslant c|h|\left[\left(\gamma^{2}+\varrho^{-2}\right) \int_{Q_{2 \varrho}^{+}}\left(1+|D u|^{2}\right) d z+\|G\|_{\mathcal{N} 0, \vartheta ; 2}^{2}\left(Q_{2 \varrho}^{+}\right)\right],
$$

where $c=c(L / v)$ and by a different choice of radii we infer the asserted estimate.

At this stage we are already able to control the time derivative $\partial_{t} u$ and all second derivatives $D_{\alpha} D_{\beta} u$ with $\alpha+$ $\beta<2 n$. Therefore, we can use the parabolic system in order to establish the existence of the second normal derivative $D_{n} D_{n} u$ together with an estimate for its $L^{2}$-norm. 
Proposition 4.10. Let $u$ be a weak solution of (4.1) on $Q_{R}^{+}$according to Definition 4.1 and let $G \in \mathcal{N}^{0, \vartheta ; 2}\left(Q_{R}^{+} ; \mathbb{R}^{N}\right)$. Then, $D u$ is weakly differentiable with respect to $x_{n}$ on $Q_{R}^{+}$with $D_{n} D u \in L^{2}\left(Q_{r}^{+}, \mathbb{R}^{N n}\right)$ for all $0<r<R$. Moreover, for any $0<\varrho \leqslant R$ we have

$$
\sup _{t \in \Lambda_{(\varrho / 2)^{2}}} \int_{B_{\varrho / 2}^{+}}\left|D_{n} u(\cdot, t)\right|^{2} d x+\int_{Q_{\varrho / 2}^{+}}\left|D_{n} D u\right|^{2} d z \leqslant c\left[\left(\gamma^{2}+\varrho^{-2}\right) \int_{Q_{\varrho}^{+}}\left(1+|D u|^{2}\right) d z+\|G\|_{\mathcal{N}^{0, \vartheta ; 2}\left(Q_{\varrho}^{+}\right)}^{2}\right],
$$

where $c=c(n, N, L / \nu, \vartheta)$ and $c \rightarrow \infty$ when $\vartheta \downarrow 0$.

Proof. Let $h \in(0, \varrho / 2)$. Similarly to the estimates for the tangential directions in the proof of Proposition 4.3 we decompose

$$
\tau_{h}^{n}[a(\cdot, D u(\cdot))](x, t)=\mathcal{A}(h)(x, t)+\mathcal{B}(h)(x, t),
$$

where we have set

$$
\begin{aligned}
\mathcal{A}(h)(x, t) & :=a\left(x+h e_{n}, t, D u\left(x+h e_{n}, t\right)\right)-a\left(x+h e_{n}, t, D u(x, t)\right) \\
& =\int_{0}^{1} \partial_{w} a\left(x+h e_{n}, t, D u(x, t)+s \tau_{h}^{n}[D u](x, t)\right) d s \tau_{h}^{n}[D u](x, t)
\end{aligned}
$$

and $\mathcal{B}(h)(x, t):=a\left(x+h e_{n}, t, D u(x, t)\right)-a(x, t, D u(x, t))$. From the ellipticity assumption $(4.2)_{2}$ we infer that

$$
\begin{aligned}
v\left|\tau_{h}^{n}[D u](x, t)\right|^{2} & \leqslant \mathcal{A}(h)(x, t) \cdot \tau_{h}^{n}[D u](x, t) \\
& =\sum_{\alpha=1}^{n} \tau_{h}^{n}\left[a_{\alpha}(\cdot, D u)\right](x, t) \cdot \tau_{h}^{n}\left[D_{\alpha} u\right](x, t)-\mathcal{B}(h)(x, t) \cdot \tau_{h}^{n}[D u](x, t) .
\end{aligned}
$$

Using (4.2) $)_{1}$ and (4.2) 3 in order to estimate the right-hand side in (4.16) we obtain

$$
\left|\tau_{h}^{n}\left[a_{\alpha}(\cdot, D u)\right]\right| \leqslant|\mathcal{A}(h)|+|\mathcal{B}(h)| \leqslant L\left(\left|\tau_{h}^{n}[D u]\right|+\gamma h(1+|D u|)\right) .
$$

Inserting this for $\alpha=1, \ldots, n-1$ above, using once again that $|\mathcal{B}(h)| \leqslant \gamma h(1+|D u|)$ and finally applying Young's inequality with $\varepsilon>0$ we deduce

$$
v\left|\tau_{h}^{n}[D u]\right|^{2} \leqslant \varepsilon\left|\tau_{h}^{n}[D u]\right|^{2}+c_{\varepsilon}\left[\sum_{\alpha=1}^{n-1}\left|\tau_{h}^{n}\left[D_{\alpha} u\right]\right|^{2}+\left|\tau_{h}^{n}\left[a_{n}(\cdot, D u)\right]\right|^{2}+\gamma^{2} h^{2}(1+|D u|)^{2}\right],
$$

where $c_{\varepsilon}=c(n, L, 1 / \varepsilon)$. Choosing $\varepsilon=v / 2$ in order to absorb $\varepsilon\left|\tau_{h}^{n}[D u]\right|^{2}$ on the left side and integrating the resulting pointwise estimate over $Q_{\varrho / 2}^{+}$then yields

$$
\int_{Q_{\varrho / 2}^{+}}\left|\tau_{h}^{n}[D u]\right|^{2} d z \leqslant c \sum_{\alpha=1}^{n-1} \int_{Q_{\varrho / 2}^{+}}\left|\tau_{h}^{n}\left[D_{\alpha} u\right]\right|^{2} d z+c \int_{Q_{\varrho / 2}^{+}}\left|\tau_{h}^{n}\left[a_{n}(\cdot, D u)\right]\right|^{2} d z+c \gamma^{2} h^{2} \int_{Q_{\varrho / 2}^{+}}(1+|D u|)^{2} d z,
$$

where $c=c(n, L / v)$. By Proposition 4.3 we already know $D_{n} D_{\alpha} u \in L^{2}\left(Q_{3 \varrho / 4}^{+}, \mathbb{R}^{n}\right)$ for $\alpha=1, \ldots, n-1$ and therefore the standard $L^{2}$-estimate for difference quotients implies

$$
\int_{Q_{\varrho / 2}^{+}}\left|\tau_{h}^{n}\left[D_{\alpha} u\right]\right|^{2} d z \leqslant h^{2} \int_{Q_{3 \varrho / 4}^{+}}\left|D_{n} D_{\alpha} u\right|^{2} d z
$$

for any $h \in(0, \varrho / 4)$. From the facts that $u$ is a solution of our parabolic system (4.1) and that $D_{\alpha} D_{\beta} u, \alpha+\beta<2 n$, and $\partial_{t} u$ are $L^{2}$-functions we find that $D_{n} a_{n}(\cdot, D u) \in L^{2}\left(Q_{3 \varrho / 4}^{+}, \mathbb{R}^{n}\right)$. Indeed, rewriting (4.1) as follows

$$
D_{n} a_{n}(\cdot, D u)=-\sum_{\alpha=1}^{n-1} D_{\alpha} a_{\alpha}(\cdot, D u)+u_{t}-G
$$


we see that Propositions 4.3 and 4.8 imply that the right-hand side of this identity is in $L^{2}\left(Q_{3 \varrho / 4}^{+} ; \mathbb{R}^{N}\right)$. Therefore, the standard $L^{2}$-estimate for difference quotients applied to $\frac{1}{h} \tau_{h}^{n}\left[a_{n}(\cdot, D u)\right]$ yields

$$
\begin{aligned}
\int_{Q_{\varrho / 2}^{+}}\left|\tau_{h}^{n}\left[a_{n}(\cdot, D u)\right]\right|^{2} d z & \leqslant h^{2} \int_{Q_{3 \varrho / 4}^{+}}\left|D_{n} a(\cdot, D u)\right|^{2} d z \\
& \leqslant c(n) h^{2} \int_{Q_{3 \varrho / 4}^{+}}\left[\sum_{\alpha=1}^{n-1}\left|D_{\alpha} a_{\alpha}(\cdot, D u)\right|^{2}+\left|u_{t}\right|^{2}+|G|^{2}\right] d z .
\end{aligned}
$$

Moreover, using again the bound on $\partial_{w} a(x, t, w)$ from (4.2) $)_{1}$ and the Lipschitz continuity of $x \mapsto a(x, t, w)$ from (4.2) 3 we obtain for $\alpha=1, \ldots, n-1,\left|D_{\alpha} a_{\alpha}(\cdot, D u)\right| \leqslant L\left(\left|D_{\alpha} D u\right|+\gamma(1+|D u|)\right)$. Now, we insert this into the sum in (4.19), and then join the result with (4.18) and (4.17). This leads us to

$$
\begin{aligned}
\int_{Q_{\varrho / 2}^{+}}\left|\tau_{h}^{n}[D u]\right|^{2} d z & \leqslant c h^{2} \int_{Q_{3 \varrho / 4}^{+}}\left[\sum_{\alpha=1}^{n-1}\left|D_{\alpha} D u\right|^{2}+\left|u_{t}\right|^{2}+\gamma^{2}(1+|D u|)^{2}+|G|^{2}\right] d z \\
& \leqslant c h^{2}\left[\left(\varrho^{-2}+\gamma^{2}\right) \int_{Q_{\varrho}^{+}}\left(1+|D u|^{2}\right) d z+\|G\|_{\mathcal{N}^{0, \vartheta ; 2}\left(Q_{\varrho}^{+}\right)}^{2}\right] .
\end{aligned}
$$

In the last line we have used Propositions 4.3 and 4.8 (with radii $3 \varrho / 4, \varrho$ instead of $\varrho / 2, \varrho$, which can be achieved as usual by a different choice of the involved cut-off functions and cylinders). Since this estimate holds for any choice of $h \in(0, \varrho / 4)$ we conclude that $D_{n} D u \in L^{2}\left(Q_{\varrho / 2}^{+} ; \mathbb{R}^{N}\right)$ with the estimate

$$
\int_{Q_{\varrho / 2}^{+}}\left|D_{n} D u\right|^{2} d z \leqslant c\left[\left(\varrho^{-2}+\gamma^{2}\right) \int_{Q_{\varrho}^{+}}\left(1+|D u|^{2}\right) d z+\|G\|_{\mathcal{N}^{0, \vartheta ; 2}\left(Q_{\varrho}^{+}\right)}^{2}\right] .
$$

Once again a different choice of the involved radii yields that $D_{n} D u \in L^{2}\left(Q_{r}^{+}, \mathbb{R}^{N n}\right)$ for any $0<r<R$. Finally, we turn our attention to the $L^{2}$-estimate for $D_{n} u(\cdot, t)$. For this aim we consider $t \in \Lambda_{(\varrho / 2)^{2}}$ and choose cut-off functions $\eta \in C_{0}^{\infty}\left(B_{3 \varrho / 4}\right)$ with $0 \leqslant \eta \leqslant 1, \eta=1$ on $B_{\varrho / 2},|D \eta| \leqslant c / \varrho$ and $\zeta \in C^{1}(\mathbb{R})$ with $0 \leqslant \zeta \leqslant 1, \zeta \equiv 0$ on $\left(-\infty,-(3 \varrho / 4)^{2}\right), \zeta \equiv 1$ on $\Lambda_{(\varrho / 2)^{2}}$ and $\left|\zeta^{\prime}\right| \leqslant c / \varrho^{2}$. For the Steklov-mean $u_{\lambda}$ with $\lambda \in(0, \varrho / 4)$ we know that $\partial_{t} D_{n} u_{\lambda} \in L^{2}\left(Q_{3 \varrho / 4}^{+}, \mathbb{R}^{N}\right)$ and $D_{n} u_{\lambda}(\cdot, t) \in L^{2}\left(Q_{3 \varrho / 4}^{+}, \mathbb{R}^{N}\right)$. Therefore, the following computation is justified:

$$
\begin{aligned}
\frac{1}{2} \int_{B_{\varrho}^{+}}\left|D_{n} u_{\lambda}(\cdot, t)\right|^{2} \eta^{2} d x & =\frac{1}{2} \int_{-\varrho^{2} \int_{\varrho}^{+}}^{t} \partial_{\tau}\left(\left|D_{n} u_{\lambda}\right|^{2} \zeta\right) \eta^{2} d x d \tau \\
& =\frac{1}{2} \int_{-\varrho^{2}}^{t} \int_{B_{\varrho}^{+}}\left(\partial_{\tau}\left|D_{n} u_{\lambda}\right|^{2} \zeta \eta^{2}+\left|D_{n} u_{\lambda}\right|^{2} \zeta^{\prime} \eta^{2}\right) d x d \tau \\
& =\int_{-\varrho^{2} B_{\varrho}^{+}}^{t}\left(D_{n}\left(\partial_{\tau} u_{\lambda}\right) \cdot D_{n} u_{\lambda} \zeta \eta^{2}+\frac{1}{2}\left|D_{n} u_{\lambda}\right|^{2} \zeta^{\prime} \eta^{2}\right) d x d \tau
\end{aligned}
$$

In the first integral on the right-hand side we integrate by parts, taking into account that $\partial_{\tau} u_{\lambda}(\cdot, \tau) \cdot D_{n} u_{\lambda}(\cdot, \tau) \eta^{2}=0$ on $\partial B_{\varrho}^{+}$. Applying also Young's inequality this yields

$$
\int_{-\varrho^{2}}^{t} \int_{B_{\varrho}^{+}} D_{n}\left(\partial_{\tau} u_{\lambda}\right) \cdot D_{n} u_{\lambda} \zeta \eta^{2} d x d \tau=\int_{-\varrho^{2}}^{t} \int_{B_{\varrho}^{+}} \partial_{\tau} u_{\lambda} \cdot D_{n}\left(D_{n} u_{\lambda} \eta^{2}\right) \zeta d x d \tau
$$




$$
\begin{aligned}
& =\int_{-\varrho^{2}}^{t} \int_{B_{\varrho}^{+}}\left(\partial_{\tau} u_{\lambda} \cdot D_{n} D_{n} u_{\lambda} \eta^{2} \zeta+\partial_{\tau} u_{\lambda} \cdot D_{n} u_{\lambda} D_{n} \eta^{2} \zeta\right) d x d \tau \\
& \leqslant c \int_{Q_{\varrho}^{+}}\left(\left|\partial_{\tau} u_{\lambda}\right|^{2}+\left|D_{n} D_{n} u_{\lambda}\right|^{2}+\varrho^{-2}\left|D_{n} u_{\lambda}\right|^{2}\right) \eta \zeta d z .
\end{aligned}
$$

Inserting this above and recalling that $\left|\zeta^{\prime}\right| \leqslant c / \varrho^{2}$ yields

$$
\frac{1}{2} \int_{B_{\varrho}^{+}}\left|D_{n} u_{\lambda}(\cdot, t)\right|^{2} \eta^{2} d x \leqslant c \int_{Q_{\varrho}^{+}}\left(\left|\partial_{\tau} u_{\lambda}\right|^{2}+\left|D_{n} D_{n} u_{\lambda}\right|^{2}+\varrho^{-2}\left|D_{n} u_{\lambda}\right|^{2}\right) \eta \zeta d z .
$$

Passing to the limit $\lambda \downarrow 0$ and recalling that $\eta \equiv 1$ on $B_{\varrho / 2}$ and spt $\eta \zeta \subset Q_{3 \varrho / 4}$, we find that for a.e. $t \in \Lambda_{(\varrho / 2)^{2}}$ there holds

$$
\begin{aligned}
\frac{1}{2} \int_{B_{\varrho / 2}^{+}}\left|D_{n} u(\cdot, t)\right|^{2} d x & \leqslant c \int_{Q_{3 \varrho / 4}^{+}}\left(\left|\partial_{\tau} u\right|^{2}+\left|D_{n} D_{n} u\right|^{2}+\varrho^{-2}\left|D_{n} u\right|^{2}\right) d z \\
& \leqslant c\left[\left(\varrho^{-2}+\gamma^{2}\right) \int_{Q_{\varrho}^{+}}\left(1+|D u|^{2}\right) d z+\|G\|_{\left.\mathcal{N}^{0, \vartheta ; 2}\left(Q_{\varrho}^{+}\right)\right]}^{2}\right.
\end{aligned}
$$

where we have used in the last line Proposition 4.8 and the estimate from above for the $L^{2}$-norm of $\left|D_{n} D_{n} u\right|$ (again with slightly changed radii $3 \varrho / 4, \varrho$ instead of $\varrho / 2, \varrho)$. Taking the supremum over $t \in \Lambda_{(\varrho / 2)^{2}}$ we obtain the desired sup-estimate. This finishes the proof of the lemma.

Lemma 4.11. Let $u$ be a weak solution of (4.1) on $Q_{R}^{+}$according to Definition 4.1 and let $G \in \mathcal{N}^{0, \vartheta ; 2}\left(Q_{\varrho}^{+} ; \mathbb{R}^{N}\right)$. Then, Du $\in L^{2+\frac{4}{n}}\left(Q_{r}^{+} ; \mathbb{R}^{N n}\right)$ for all $0<r<R$. Moreover, for any $0<\varrho \leqslant R$ there holds

$$
\int_{Q_{\varrho / 2}^{+}}|D u|^{2+\frac{4}{n}} d z \leqslant c\left[\left(\varrho^{-2}+\gamma^{2}\right) \int_{Q_{\varrho}^{+}}\left(1+|D u|^{2}\right) d z+\|G\|_{\mathcal{N}^{0, \vartheta ; 2}\left(Q_{\varrho}^{+}\right)}^{2}\right]^{1+\frac{2}{n}},
$$

where $c=c(n, N, L / \nu, \vartheta)$ and $c \rightarrow \infty$ when $\vartheta \downarrow 0$.

Proof. We choose a cut-off function $\eta \in C_{0}^{\infty}\left(B_{3 \varrho / 4}\right)$ with $0 \leqslant \eta \leqslant 1, \eta \equiv 1$ on $B_{\varrho / 2}$ and $|D \eta| \leqslant c / \varrho$. From Proposition 4.10 we know that $\left|D^{2} u\right| \in L^{2}\left(Q_{3 \varrho / 4}^{+}\right)$. This allows us to apply the boundary version of the Sobolev-embedding from Lemma 2.3 slice wise on $B_{t}^{+}=B_{3 \varrho / 4}^{+} \times\{t\}$ for $t \in \Lambda_{(3 \varrho / 4)^{2}}$, yielding that

$$
\begin{aligned}
\int_{B_{t}^{+}}|D u|^{2+\frac{4}{n}} \eta^{2} d x & =\int_{B_{t}^{+}}\left(|D u|^{1+\frac{2}{n}} \eta\right)^{2} d x \\
& \leqslant c\left(\int_{B_{t}^{+}}\left|D\left(|D u|^{1+\frac{2}{n}} \eta\right)\right|^{\frac{2 n}{n+2}} d x\right)^{\frac{n+2}{n}} \\
& \leqslant c\left(\int_{B_{t}^{+}}\left(\left.|D| D u\right|^{1+\frac{2}{n}} \mid \eta\right)^{\frac{2 n}{n+2}} d x\right)^{\frac{n+2}{n}}+c\left(\int_{B_{t}^{+}}|D u|^{2}|D \eta|^{\frac{2 n}{n+2}} d x\right)^{\frac{n+2}{n}} \\
& =: c I(t)+c I I(t),
\end{aligned}
$$

where $c=c(n)$. In the sequel we estimate $I(t)$ and $I I(t)$. We start by considering $I(t)$. First, we note that 


$$
\left.\left.|D| D u\right|^{1+\frac{2}{n}}\left|=\frac{n+2}{n}\right| D u\right|^{\frac{2}{n}}|D| D u|| \leqslant \frac{n+2}{n}|D u|^{\frac{2}{n}}\left|D^{2} u\right|,
$$

and using Hölder's inequality we obtain

$$
I(t) \leqslant c\left(\int_{B_{t}^{+}}|D u|^{\frac{4}{n+2}}\left|D^{2} u\right|^{\frac{2 n}{n+2}} d x\right)^{\frac{n+2}{n}} \leqslant c\left(\int_{B_{t}^{+}}|D u|^{2} d x\right)^{\frac{2}{n}} \cdot \int_{B_{t}^{+}}\left|D^{2} u\right|^{2} d x .
$$

We now turn our attention to $I I(t)$. Using $|D \eta| \leqslant c / \varrho$ we find that

$$
I I(t) \leqslant c \varrho^{-2}\left(\int_{B_{t}^{+}}|D u|^{2} d x\right)^{\frac{2}{n}} \cdot \int_{B_{t}^{+}}|D u|^{2} d x .
$$

We now integrate over $t \in \Lambda_{(\varrho / 2)^{2}}$, note that $\eta \equiv 1$ on $B_{\varrho / 2}$ and insert the estimates for $I(t)$ and $I I(t)$ above. This leads us to

$$
\begin{aligned}
\int_{Q_{\varrho / 2}^{+}}|D u|^{2+\frac{4}{n}} d z & \leqslant c\left(\sup _{t \in \Lambda_{(\varrho / 2)^{2}}} \int_{B_{3 \varrho / 4}^{+}}|D u(\cdot, t)|^{2} d x\right)^{\frac{2}{n}} \int_{Q_{3 \varrho / 4}^{+}}\left|D^{2} u\right|^{2}+\varrho^{-2}|D u|^{2} d z \\
& \leqslant c\left[\left(\varrho^{-2}+\gamma^{2}\right) \int_{Q_{\varrho}^{+}}\left(1+|D u|^{2}\right) d z+\|G\|_{\mathcal{N}^{0, \vartheta ; 2}\left(Q_{\varrho}^{+}\right)}^{2}\right]^{1+\frac{2}{n}},
\end{aligned}
$$

where we have applied Propositions 4.3 and 4.10 in the last line (for radii $3 \varrho / 4, \varrho$ instead of $\varrho / 2, \varrho$ ). Once again we can also achieve $D u \in L^{2+\frac{4}{n}}\left(Q_{r}^{+} ; \mathbb{R}^{n}\right)$ for any $0<r<R$ by a different choice of radii.

\subsubsection{Higher integrability}

Here, we are concerned with the higher integrability properties of the time derivative, respectively second-order space derivatives up to the lateral boundary of weak solutions of non-linear differentiable systems as considered in (4.1). For this aim we will have to go through the sequence of the lemmas obtained in Section 4.1 and push them to a higher exponent. We will start by showing the higher integrability of $\tau_{h} D u$. Here, we shall use the fact that $h^{-\frac{1}{2}} \tau_{h} D u$ solves a linear parabolic system. This procedure results in the following integrability improvement of Lemma 4.5:

Lemma 4.12. Let $u$ be a weak solution of (4.1) on $Q_{R}^{+}$according to Definition 4.1 and suppose that $G \in L^{\sigma_{1}}\left(Q_{R}^{+}, \mathbb{R}^{N}\right)$ for some $\sigma_{1}>2$. Then there exists $\sigma_{2}=\sigma_{2}(n, L / v) \in\left(2, \min \left\{\sigma_{1}, 2+\frac{4}{n}\right\}\right]$ such that for all $\sigma \in\left(2, \sigma_{2}\right], \varepsilon \in(0,1]$, $Q_{\varrho}\left(z_{0}\right) \subset Q_{R}$ with $z_{0}=\left(x_{0}, t_{0}\right) \in Q_{R}^{+} \cup \Gamma_{R}$ and $0<|h| \leqslant\left(R^{2}-\varrho^{2}-\left|t_{0}\right|\right) / 2$ there holds

$$
\begin{aligned}
\int_{Q_{\varrho / 2}^{+}\left(z_{0}\right)}\left|\tau_{h} D u\right|^{\sigma} d z \leqslant & c\left(f_{Q_{\varrho}^{+}\left(z_{0}\right)}\left|\tau_{h} D u\right|^{2} d z\right)^{\frac{\sigma}{2}}+\varepsilon^{\frac{\sigma}{2}} f_{Q_{\varrho}^{+}\left(z_{0}\right)} \frac{\left|\tau_{-h} \tau_{h} u\right|^{\sigma}}{|h|^{\frac{\sigma}{2}}} d z \\
& +c|h|^{\frac{\sigma}{2}}\left[|h|^{\frac{\vartheta \sigma}{2}} \gamma^{\sigma} f_{Q_{\varrho}^{+}\left(z_{0}\right)}\left(1+|D u|^{\sigma}\right) d z+\frac{1}{\varepsilon^{\frac{\sigma}{2}}} f_{Q_{\varrho}^{+}\left(z_{0}\right)}|\mathcal{G}|^{\sigma} d z\right],
\end{aligned}
$$

where $\mathcal{G}$ is defined in Lemma 4.5 and $c=c(n, N, L / v)$.

Proof. We define $v_{h}=|h|^{-1 / 2} \tau_{h} u$. For $0<\varrho \leqslant R$ and $0<|h|<\left(R^{2}-\varrho^{2}\right) / 2$ and $\varepsilon \in(0,1]$ we obtain from Lemma 4.5 (with $\varrho / 2, \varrho$ instead of $r, \varrho$ ) the following Caccioppoli-type inequality for $v_{h}$ :

$$
\sup _{t \in \Lambda_{(\varrho / 2)^{2}}} \int_{B_{\varrho / 2}^{+}}\left|v_{h}(\cdot, t)\right|^{2} d x+\int_{Q_{\varrho / 2}^{+}}\left|D v_{h}\right|^{2} d z \leqslant c \int_{Q_{\varrho}^{+}}\left|\frac{v_{h}}{\varrho}\right|^{2} d z+\varepsilon \int_{Q_{\varrho}^{+}} \frac{\left|\tau_{-h} v_{h}\right|^{2}}{|h|} d z
$$




$$
+c \int_{Q_{\varrho}^{+}}|h|^{\vartheta} \gamma^{2}\left(1+|D u|^{2}\right)+\frac{1}{\varepsilon}|\mathcal{G}|^{2} d z
$$

where $c=c(L / v)$. Proceeding similarly to the proof of Lemma 3.1 we infer the following reverse-Hölder-type inequality

$$
\begin{aligned}
f_{Q_{\varrho / 2}^{+}}\left|D v_{h}\right|^{2} d z \leqslant & \frac{1}{2} f_{Q_{2 \varrho}^{+}}\left|D v_{h}\right|^{2} d z+c\left(f_{Q_{\varrho}^{+}}\left|D v_{h}\right|^{\frac{2 n}{n+2}} d z\right)^{\frac{n+2}{n}} \\
& +\varepsilon f_{Q_{2 \varrho}^{+}} \frac{\left|\tau_{-h} v_{h}\right|^{2}}{|h|} d z+c f_{Q_{2 \varrho}^{+}}|h|^{\vartheta} \gamma^{2}\left(1+|D u|^{2}\right)+\frac{1}{\varepsilon}|\mathcal{G}|^{2} d z,
\end{aligned}
$$

where $c=c(n, L / v)$. Note that in order to have the preceding reverse-Hölder inequality we may have to replace $\varepsilon$ by a smaller value. The derivation of the preceding estimate did by no means utilize the fact that the cylinders are centered at zero - this was just for convenience in notation. Indeed, the estimate also holds for any parabolic cylinder with center $z_{1} \subset \Gamma_{R}$ and radius $\varrho$ such that $Q_{2 \varrho+\sqrt{ }|h|}^{+}\left(z_{1}\right) \subset Q_{R}^{+}$. Moreover, a similar reverse-Hölder-type inequality holds for interior cylinders $Q_{\varrho}\left(z_{1}\right)$ satisfying $Q_{2 \varrho+\sqrt{ }|h|}\left(z_{1}\right) \subset Q_{R}^{+}$. Therefore, we are in the position where we can apply an up to the boundary version of Gehring's theorem; the argument is quite similar to the proof of Lemma 3.1. We infer the existence of a higher integrability exponent $\sigma_{2}=\sigma_{2}(n, L / \nu) \in\left(2, \min \left\{\sigma_{1}, 2+\frac{4}{n}\right\}\right]$ such that for any $\sigma \in\left(2, \sigma_{2}\right]$ there holds

$$
\begin{aligned}
f_{Q_{\varrho / 2}^{+}\left(z_{0}\right)}\left|D v_{h}\right|^{\sigma} d z \leqslant & c\left(f_{Q_{\varrho}^{+}\left(z_{0}\right)}\left|D v_{h}\right|^{2} d z\right)^{\frac{\sigma}{2}}+\varepsilon^{\frac{\sigma}{2}} f_{Q_{\varrho}^{+}\left(z_{0}\right)} \frac{\left|\tau_{-h} v_{h}\right|^{\sigma}}{|h|^{\frac{\sigma}{2}}} d z \\
& +c f_{Q_{\varrho}^{+}\left(z_{0}\right)}|h|^{\frac{\vartheta \sigma}{2}} \gamma^{\sigma}\left(1+|D u|^{\sigma}\right)+\varepsilon^{-\frac{\sigma}{2}}|\mathcal{G}|^{\sigma} d z,
\end{aligned}
$$

where $c$ depends on $n, N, L / v$ but is independent of $h$. Note, that we can always decrease the value of $\varepsilon$ if necessary. Recalling the definition of $v_{h}$ we infer the desired estimate.

Corollary 4.13. Let $u$ be a weak solution of (4.1) on $Q_{R}^{+}$according to Definition 4.1 and suppose that $G \in$ $L^{\sigma_{1}}\left(Q_{R}^{+}, \mathbb{R}^{N}\right)$ for some $\sigma_{1}>2$. Then there exists $\sigma_{2} \in\left(2, \min \left\{\sigma_{1}, 2+\frac{4}{n}\right\}\right]$ such that for all $\sigma \in\left(2, \sigma_{2}\right], \varepsilon>0$, $Q_{\varrho}\left(z_{0}\right) \Subset Q_{R}$ with $z_{0}=\left(x_{0}, t_{0}\right) \in Q_{R}^{+} \cup \Gamma_{R}$ and $0<|h| \leqslant\left(R^{2}-\varrho^{2}-\left|t_{0}\right|\right) / 2$ there holds

$$
\begin{aligned}
f_{Q_{\varrho / 2}^{+}\left(z_{0}\right)}\left|\tau_{h} D u\right|^{\sigma} d z \leqslant & c\left(f_{Q_{\varrho}^{+}\left(z_{0}\right)}\left|\tau_{h} D u\right|^{2} d z\right)^{\frac{\sigma}{2}}+\frac{c}{\varepsilon^{\frac{\sigma}{2}}} f_{Q_{\varrho}^{+}\left(z_{0}\right)}\left|\tau_{h} u\right|^{\sigma} d z \\
& +c|h|^{\frac{(1+\vartheta) \sigma}{2}} \gamma^{\sigma} f_{Q_{\varrho}^{+}\left(z_{0}\right)}\left(1+|D u|^{\sigma}\right) d z+\varepsilon^{\frac{\sigma}{2}} f_{Q_{\varrho}^{+}\left(z_{0}\right)}\left|\tau_{h} G\right|^{\sigma} d z .
\end{aligned}
$$

Proof. Starting from Corollary 4.6 instead of Lemma 4.5 the argument from the proof of Lemma 4.12 yields the asserted estimate.

Lemma 4.14. Let $u$ be a weak solution of (4.1) on $Q_{R}^{+}$according to Definition 4.1 and assume that $G \in L^{\sigma_{1}}\left(Q_{R}^{+}, \mathbb{R}^{N}\right)$ for some $\sigma_{1}>2$. Then for all parabolic cylinders $Q_{\varrho}^{+}\left(z_{0}\right)$ with $0<\varrho \leqslant R, z_{0}=\left(x_{0}, t_{0}\right) \in Q_{R}^{+} \cup \Gamma_{R}, \sigma \in$ $\left(2, \min \left\{\sigma_{1}, 2+\frac{4}{n}\right\}\right]$ and $0<|h| \leqslant\left(R^{2}-\varrho^{2}-\left|t_{0}\right|\right) / 2$ there holds

$$
f_{Q_{\varrho / 2}^{+}\left(z_{0}\right)} \frac{\left|\tau_{-h} \tau_{h} u\right|^{\sigma}}{|h|^{\frac{\sigma}{2}}} d z \leqslant c\left(f_{Q_{\varrho / 2}^{+}\left(z_{0}\right)} \frac{\left|\tau_{-h} \tau_{h} u\right|^{2}}{|h|} d z\right)^{\frac{\sigma}{2}}+c \underset{Q_{\varrho}^{+}\left(z_{0}\right)}{f}\left|\tau_{h} D u\right|^{\sigma}+\left|\tau_{-h} D u\right|^{\sigma} d z
$$




$$
+c|h|^{\frac{\sigma}{2}} f_{Q_{\varrho}^{+}\left(z_{0}\right)}|h|^{\frac{\vartheta \sigma}{2}} \gamma^{\sigma}\left(1+|D u|^{\sigma}\right)+\left|\tau_{h} G\right|^{\sigma}+\left|\tau_{-h} G\right|^{\sigma} d z
$$

where $c=c(n, N, L, \sigma)$ and $c \rightarrow \infty$ when $\sigma \downarrow 2$.

Proof. We let $\sigma_{2}=\min \left\{\sigma_{1}, 2+\frac{4}{n}\right\}$ and consider a fixed parabolic cylinder of the type $Q_{r}\left(z_{1}\right) \subset Q_{\varrho / 2}^{+}\left(z_{0}\right)$ with $z_{1}=\left(x_{1}, t_{1}\right)$. In the sequel we distinguish the cases $0<|h| \leqslant(r / 2)^{2}$ and $|h|>(r / 2)^{2}$. In the case $|h| \leqslant(r / 2)^{2}$ we apply Lemma 4.7 with $(r, 3 r / 2)$ instead of $(r, \varrho)$ to infer that

$$
\begin{aligned}
f_{Q_{r}\left(z_{1}\right)}\left|\tau_{-h} \tau_{h} u\right|^{2} d z \leqslant c|h| & f_{Q_{2 r}^{+}\left(z_{1}\right)}\left|\tau_{h} D u\right|^{2}+\left|\tau_{-h} D u\right|^{2} d z \\
& +c h^{2} \int_{Q_{2 r}^{+}\left(z_{1}\right)}|h|^{\vartheta} \gamma^{2}\left(1+|D u|^{2}\right)+\left|\tau_{h} G\right|^{2}+\left|\tau_{-h} G\right|^{2} d z,
\end{aligned}
$$

where $c=c(n, N, L)$. Note that we applied Lemma 4.7 in a situation where $Q_{r}^{+}\left(z_{1}\right)=Q_{r}\left(z_{1}\right)$ which is possible by the hypothesis of the lemma. In the case $|h|>(r / 2)^{2}$ we choose a non-negative weight-function $\eta \in C_{0}^{\infty}\left(B_{r}\left(x_{1}\right)\right)$ with $f_{B_{r}\left(x_{1}\right)} \eta d x=1,0 \leqslant \eta \leqslant c$ and $|D \eta| \leqslant c / r$. Then, the weighted means $\left(\tau_{-h} \tau_{h} u\right)_{\eta}(t)$ of $\tau_{-h} \tau_{h} u$ are defined via averaging by $\left(\tau_{-h} \tau_{h} u\right)_{\eta}(t):=f_{B_{r}\left(x_{1}\right)} \tau_{-h} \tau_{h} u(\cdot, t) \eta d x$. The mean square deviation of $\tau_{-h} \tau_{h} u$ on $Q_{r}\left(z_{1}\right)$ from its mean value can then be decomposed as follows:

$$
\begin{aligned}
& f_{\Lambda_{r^{2}}\left(t_{1}\right)} f_{B_{r}\left(x_{1}\right)}\left|\tau_{-h} \tau_{h} u-\left(\tau_{-h} \tau_{h} u\right)_{z_{1}, r}\right|^{2} d x d t \leqslant 3\left[f_{\Lambda_{r^{2}}\left(t_{1}\right)} \underset{B_{r}\left(x_{1}\right)}{f}\left|\tau_{-h} \tau_{h} u(x, t)-\left(\tau_{-h} \tau_{h} u\right)_{\eta}(t)\right|^{2} d x d t\right. \\
& +f_{\Lambda_{r^{2}\left(t_{1}\right)}}\left|\left(\tau_{-h} \tau_{h} u\right)_{\eta}(t)-f_{\Lambda_{r^{2}}\left(t_{1}\right)}\left(\tau_{-h} \tau_{h} u\right)_{\eta}(\tau) d \tau\right|^{2} d t \\
& \left.+\left|f_{\Lambda_{r^{2}}\left(t_{1}\right)}\left(\tau_{-h} \tau_{h} u\right)_{\eta}(\tau) d \tau-\left(\tau_{-h} \tau_{h} u\right)_{z_{1}, r}\right|^{2} d t\right] \\
& =: 3(I+I I+I I I),
\end{aligned}
$$

with the obvious meaning of $I-I I I$. To estimate $I$ we apply Poincaré's inequality slice wise for a.e. $t \in \Lambda_{r^{2}}\left(t_{1}\right)$ and use the fact that $r^{2}<4|h|$ to deduce

$$
I \leqslant c r^{2} f_{\Lambda_{r^{2}}\left(t_{1}\right)} f_{B_{r}\left(x_{1}\right)}\left|D\left(\tau_{-h} \tau_{h} u\right)\right|^{2} d x d t \leqslant c|h| f_{Q_{r}\left(z_{1}\right)}\left|\tau_{h} D u\right|^{2}+\left|\tau_{-h} D u\right|^{2} d z,
$$

where $c=c(n)$. Here we have used once again (2.8). Similarly, we obtain for III

$$
I I I \leqslant f_{\Lambda_{r^{2}}\left(t_{1}\right)} f_{B_{r}\left(x_{1}\right)}\left|\left(\tau_{-h} \tau_{h} u\right)_{\eta}(\tau)-\tau_{-h} \tau_{h} u(x, \tau)\right|^{2} d x d \tau=I \leqslant c|h| f_{Q_{r}\left(z_{1}\right)}\left|\tau_{h} D u\right|^{2}+\left|\tau_{-h} D u\right|^{2} d z,
$$

where $c$ depends only on $n$. We now consider the integral $I I$ whose estimation is more involved. We define for a fixed $i \in\{1, \ldots, N\}$ the test-function $\varphi: \mathbb{R}^{n+1} \rightarrow \mathbb{R}^{N}$ by letting $\varphi_{i}=\eta$ and $\varphi_{j}=0$ for $j \neq i$. Testing the Steklov formulation (4.3) (here we first replace the parameter $h$ in the Steklov formulation by $\lambda$ ) at levels $s$ and $s+h$ with the test-function $\varphi$ and taking the difference of the resulting equations yields after integrating the result with respect to $s$ over $(\tau, t)$ the following identity:

$$
\left(\tau_{h}\left[u_{i}\right]_{\lambda}\right)_{\eta}(t)-\left(\tau_{h}\left[u_{i}\right]_{\lambda}\right)_{\eta}(\tau)=\int_{\tau}^{t} \partial_{s}\left(\tau_{h}\left[u_{i}\right]_{\lambda}\right)_{\eta} d s=-\int_{\tau}^{t} f_{B_{r}\left(x_{1}\right)}\left\langle\tau_{h}\left[a_{i}(\cdot, D u)\right]_{\lambda}, D \eta\right\rangle-\left\langle\tau_{h}\left[G_{i}\right]_{\lambda}, \eta\right\rangle d x d s .
$$


In the preceding identity we pass to the limit $\lambda \downarrow 0$. Using the assumptions (1.2) 1 and (1.2) 3 on the vector field $a$ (similarly to the proof of Lemma 4.7) we see that

$$
\left|\tau_{h}\left[a_{i}(\cdot, D u)\right](x, t)\right| \leqslant L\left|\tau_{h}[D u](x, t)\right|+L \gamma|h|^{\frac{1+\vartheta}{2}}(1+|D u(x, t)|) .
$$

Inserting this above and summing over $i=1, \ldots, N$ leads us to

$$
\left|\left(\tau_{h} u\right)_{\eta}(t)-\left(\tau_{h} u\right)_{\eta}(\tau)\right| \leqslant c L r \int_{Q_{r}\left(z_{1}\right)}\left|\tau_{h} D u\right|+\gamma|h|^{\frac{1+\vartheta}{2}}(1+|D u|) d z+c r^{2} f_{Q_{r}\left(z_{1}\right)}\left|\tau_{h} G\right| d z,
$$

where $c=c(n, N)$. Note that the preceding estimate also holds for $h$ replaced by $-h$. Therefore, using the previous estimate for $\tau_{h} u$ and $\tau_{-h} u$ and taking into account $r \leqslant 2|h|^{\frac{1}{2}}$ as well as $\left|\tau_{h} f(t-h)\right|=\left|\tau_{-h} f(t)\right|$ we obtain for a.e. $t, \tau \in \Lambda_{r^{2}}\left(t_{1}\right)$ that

$$
\begin{aligned}
& \left|\left(\tau_{-h} \tau_{h} u\right)_{\eta}(t)-\left(\tau_{-h} \tau_{h} u\right)_{\eta}(\tau)\right| \\
& \quad \leqslant\left|\left(\tau_{h} u\right)_{\eta}(t-h)-\left(\tau_{h} u\right)_{\eta}(\tau-h)\right|+\left|\left(\tau_{h} u\right)_{\eta}(t)-\left(\tau_{h} u\right)_{\eta}(\tau)\right| \\
& \quad=\left|\left(\tau_{-h} u\right)_{\eta}(t)-\left(\tau_{-h} u\right)_{\eta}(\tau)\right|+\left|\left(\tau_{h} u\right)_{\eta}(t)-\left(\tau_{h} u\right)_{\eta}(\tau)\right| \\
& \quad \leqslant c|h|^{\frac{1}{2}} f_{Q_{r}\left(z_{1}\right)}\left|\tau_{h} D u\right|+\left|\tau_{-h} D u\right| d z+c|h| f_{Q_{r}\left(z_{1}\right)}|h|^{\frac{\vartheta}{2}} \gamma(1+|D u|)+\left|\tau_{h} G\right|+\left|\tau_{-h} G\right| d z .
\end{aligned}
$$

We integrate this over $\Lambda_{r^{2}}\left(t_{1}\right)$ with respect to $t$ and $\tau$ and then use Hölder's inequality to obtain

$$
I I \leqslant f_{\Lambda_{r^{2}}\left(t_{1}\right)} f_{\Lambda_{r^{2}}\left(t_{1}\right)}\left|\left(\tau_{-h} \tau_{h} u\right)_{\eta}(t)-\left(\tau_{-h} \tau_{h} u\right)_{\eta}(\tau)\right|^{2} d \tau d t \leqslant c f_{Q_{r}\left(z_{1}\right)}\left|w_{h}\right|^{2} d z,
$$

where $c=c(n, N, L)$ and we have defined

$$
w_{h}:=|h|^{\frac{1}{2}}\left(\left|\tau_{h} D u\right|+\left|\tau_{-h} D u\right|\right)+|h|\left[|h|^{\frac{\vartheta}{2}} \gamma(1+|D u|)+\left|\tau_{h} G\right|+\left|\tau_{-h} G\right|\right]
$$

Joining the estimates obtained so far for $I-I I I$ with (4.20) and enlarging the radius from $r$ to $2 r$ we arrive at

$$
f_{Q_{r}\left(z_{1}\right)}\left|\tau_{-h} \tau_{h} u-\left(\tau_{-h} \tau_{h} u\right)_{z_{1}, r}\right|^{2} d z \leqslant c f_{Q_{2 r}^{+}\left(z_{1}\right)}\left|w_{h}\right|^{2} d z
$$

where $w_{h} \in L^{\sigma_{2}}\left(Q_{\varrho}^{+}\left(z_{0}\right)\right)$ is defined in (4.21) and $c=c(n, N, L)$. We note that (4.22) also holds in the first case.

Our next goal is to derive an upper bound for the sharp maximal function of $\tau_{-h} \tau_{h} u$. For this we consider a point $\bar{z} \in Q_{\varrho / 2}^{+}\left(z_{0}\right)$ and an arbitrary parabolic cylinder $Q_{r}\left(z_{1}\right) \subset Q_{\varrho / 2}^{+}\left(z_{0}\right)$ such that $\bar{z} \in Q_{r}\left(z_{1}\right)$. With Hölder's inequality we obtain from the preceding estimate

$$
\begin{aligned}
\left(\int_{Q_{r}\left(z_{1}\right)}\left|\tau_{-h} \tau_{h} u-\left(\tau_{-h} \tau_{h} u\right)_{z_{1}, r}\right| d z\right)^{2} & \leqslant \int_{Q_{r}\left(z_{1}\right)}\left|\tau_{-h} \tau_{h} u-\left(\tau_{-h} \tau_{h} u\right)_{z_{1}, r}\right|^{2} d z \\
& \leqslant c \int_{Q_{2 r}^{+}\left(z_{1}\right)}\left|w_{h}\right|^{2} d z \\
& \leqslant c \sup _{Q_{\hat{r}}^{+}(\hat{z}) \subset Q_{\varrho}^{+}\left(z_{0}\right), \bar{z} \in Q_{\hat{r}}^{+}(\hat{z})} \underset{Q_{\hat{r}}^{+}(\hat{z})}{f}\left|w_{h}\right|^{2} d z \\
& \leqslant c M\left(\left|w_{h}\right|^{2} \chi_{Q_{\varrho}^{+}\left(z_{0}\right)}\right)(\bar{z}),
\end{aligned}
$$

where $c=c(n, N, L)$. Here $M(f)$ denotes for an integrable function $f: \mathbb{R}^{n+1} \rightarrow \mathbb{R}$ and $\bar{z} \in \mathbb{R}^{n+1}$ the maximal function on $\mathbb{R}^{n+1}$ with respect to the parabolic cylinders, i.e.

$$
M(f)(\bar{z}):=\sup _{\bar{z} \in Q_{\hat{r}}(\hat{z})} f_{Q_{\hat{r}}(\hat{z})}|f| d z .
$$


This allows us to bound the sharp function of $\tau_{-h} \tau_{h} u$ at any point $\bar{z} \in Q_{\varrho / 2}^{+}\left(z_{0}\right)$ by taking the supremum over all parabolic cylinders $Q_{r}\left(z_{1}\right)$ with $\bar{z} \in Q_{r}\left(z_{1}\right) \subset Q_{\varrho / 2}^{+}\left(z_{0}\right)$ in the last inequality. Here, we use the local sharp maximal operator relative to the cylinder $Q_{\varrho / 2}^{+}\left(z_{0}\right)$. In this way we obtain

$$
\left[\tau_{-h} \tau_{h} u\right]_{Q_{\varrho / 2}^{+}\left(z_{0}\right)}^{\#}(\bar{z}):=\sup _{Q_{r}\left(z_{1}\right) \subset Q_{\varrho / 2}^{+}\left(z_{0}\right), \bar{z} \in Q_{r}\left(z_{1}\right)} f_{Q_{r}\left(z_{1}\right)}\left|\tau_{-h} \tau_{h} u-\left(\tau_{-h} \tau_{h} u\right)_{z_{1}, r}\right| d z \leqslant c M\left(\left|w_{h}\right|^{2} \chi_{Q_{\varrho}^{+}\left(z_{0}\right)}\right)^{\frac{1}{2}}(\bar{z}) .
$$

We integrate this over $Q_{\varrho / 2}^{+}\left(z_{0}\right)$ and then applying the Hardy-Littlewood maximal theorem to find that for any $\sigma \in$ $\left(2, \sigma_{2}\right]$ there holds

$$
\int_{Q_{\varrho / 2}^{+}\left(z_{0}\right)}\left|\left[\tau_{-h} \tau_{h} u\right]_{Q_{\varrho / 2}^{+}\left(z_{0}\right)}^{\#}\right|^{\sigma} d z \leqslant c \int_{Q_{\varrho / 2}^{+}\left(z_{0}\right)} M\left(\left|w_{h}\right|^{2} \chi_{Q_{\varrho}^{+}\left(z_{0}\right)}\right)^{\frac{\sigma}{2}} d z \leqslant c \int_{\mathbb{R}^{n+1}}\left|w_{h}\right|^{\sigma} \chi_{Q_{\varrho}^{+}\left(z_{0}\right)} d z=c \int_{Q_{\varrho}^{+}\left(z_{0}\right)}\left|w_{h}\right|^{\sigma} d z,
$$

where $c=c(n, N, L, \sigma)$. Using a theorem by C. Fefferman and E.M. Stein [20, Theorem 5] in a localized version (see [5, Chapter 5, Corollary 7.5], [24, Lemma 4]) we can bound the $L^{\sigma}$-norm of a function in terms of the $L^{\sigma}$-norm of its sharp function and the $L^{1}$-norm of the function. Precisely, we arrive at the following estimate:

$$
f_{Q_{\varrho / 2}^{+}\left(z_{0}\right)}\left|\tau_{-h} \tau_{h} u\right|^{\sigma} d z \leqslant c f_{Q_{\varrho}^{+}\left(z_{0}\right)}\left|w_{h}\right|^{\sigma} d z+c\left(f_{Q_{\varrho / 2}^{+}\left(z_{0}\right)}\left|\tau_{-h} \tau_{h} u\right| d z\right)^{\sigma},
$$

with $c=c(n, N, L)$. Recalling the definition of $w_{h}$ and applying Hölder's inequality this proves the assertion of the lemma.

Now we are in a position to prove a higher integrability result for the second derivatives $D^{2} u$ and the time derivative $\partial_{t} u$ up to the lateral boundary $\Gamma_{R}$.

Proposition 4.15. Let $u$ be a weak solution of (4.1) on $Q_{R}^{+}$according to Definition 4.1 and assume that $G \in$ $\mathcal{N}^{0, \vartheta, \sigma_{1}}\left(Q_{R}^{+} ; \mathbb{R}^{N}\right)$ for some $\sigma_{1}>2$. Then there exists $\sigma_{2}=\sigma_{2}(n, L / v) \in\left(2, \min \left\{\sigma_{1}, 2+\frac{4}{n}\right\}\right]$, such that we have $D^{2} u \in L^{\sigma_{2}}\left(Q_{r}^{+}, \mathbb{R}^{N n^{2}}\right)$ for all $0<r<R$. Moreover, for any $0<\varrho \leqslant R$ and $\sigma \in\left(2, \sigma_{2}\right]$ there holds

$$
\int_{Q_{\varrho / 2}^{+}}\left(\left|D^{2} u\right|^{\sigma}+\left|u_{t}\right|^{\sigma}\right) d z \leqslant c\left[\left(\gamma^{\sigma}+\varrho^{-\sigma}\right) \int_{Q_{\varrho}^{+}}\left(1+|D u|^{\sigma}\right) d z+\|G\|_{\mathcal{N}^{0, \vartheta ; \sigma}\left(Q_{\varrho}^{+}\right)}^{\sigma}\right],
$$

where $c=c(n, N, L / v, \vartheta, \sigma)$ and $c \rightarrow \infty$ when $\vartheta \downarrow 0$ or $\sigma \downarrow 2$.

Proof. In order to treat the tangential derivatives $D_{\alpha} D u, \alpha=1, \ldots, n-1$, we differentiate the parabolic system (4.1) with respect to $x_{\alpha}$ which is possible on behalf of Theorem 4.2. Noting that $D_{\alpha} a(x, t, D u(x, t))=\partial_{x_{\alpha}} a(x, t, D u)+$ $\partial_{w} a(x, t, D u) D_{\alpha} D u$ we infer that $D_{\alpha} u$ is a weak solution of the following linear parabolic system

$$
\int_{Q_{R}^{+}} D_{\alpha} u \cdot \varphi_{t}-\left\langle\partial_{w} a(\cdot, D u) D D_{\alpha} u, D \varphi\right\rangle d z=\int_{Q_{R}^{+}}\left\langle G, D_{\alpha} \varphi\right\rangle+\left\langle\partial_{x_{\alpha}} a(\cdot, D u), D \varphi\right\rangle d z
$$

whenever $\varphi \in C_{0}^{\infty}\left(Q_{R}^{+}, \mathbb{R}^{N}\right)$. Moreover, we have $D_{\alpha} u=0$ on $\Gamma_{R}$. From our growth and ellipticity assumptions $(4.2)_{1}$ and $(4.2)_{2}$ we know that $\left\langle\partial_{w} a(x, t, w) \tilde{w}, \tilde{w}\right\rangle \geqslant v|\tilde{w}|^{2}$ and $\left|\partial_{w} a(x, t, w)\right| \leqslant L$ for all $w, \tilde{w} \in \mathbb{R}^{N n}$. Moreover, from (4.2) 3 and Lemma 4.11 we infer that $\partial_{x_{\alpha}} a(x, t, D u) \in L^{2+\frac{n}{4}}\left(Q_{\varrho}^{+} ; \mathbb{R}^{N n}\right)$ for any $\varrho<R$ (note here that $\left.\left|\partial_{x_{\alpha}} a(x, t, D u)\right| \leqslant L \gamma(1+|D u|)\right)$. Therefore, we can apply Lemma 3.1 with a right-hand side

$$
b(x, t)=\partial_{x_{\alpha}} a(x, t, D u)+G(x, t) \otimes e_{\alpha} \in L^{\sigma_{1}}\left(Q_{\varrho}^{+} ; \mathbb{R}^{N n}\right)
$$

to infer the existence of $\tilde{\sigma}_{2}=\tilde{\sigma}_{2}(n, L / v) \in\left(2, \min \left\{\sigma_{1}, 2+\frac{4}{n}\right\}\right]$, such that $D D_{\alpha} u \in L^{\sigma}\left(Q_{\varrho / 2}^{+} ; \mathbb{R}^{N n}\right)$ for all $\sigma \in\left(2, \tilde{\sigma}_{2}\right]$. Moreover, for $0<\varrho \leqslant R$ we have 


$$
\begin{aligned}
f_{Q_{\varrho / 4}^{+}}\left|D_{\alpha} D u\right|^{\sigma} d z & \leqslant c\left(f_{Q_{\varrho / 2}^{+}}\left|D_{\alpha} D u\right|^{2} d z\right)^{\frac{\sigma}{2}}+c f_{Q_{\varrho / 2}^{+}} L^{\sigma} \gamma^{\sigma}(1+|D u|)^{\sigma}+|G|^{\sigma} d z \\
& \leqslant c(n, N, L / v)\left[\left(\gamma^{\sigma}+\varrho^{-\sigma}\right) \int_{Q_{\varrho}^{+}}\left(1+|D u|^{\sigma}\right) d z+f_{Q_{\varrho}^{+}}|G|^{\sigma} d z\right],
\end{aligned}
$$

where we have used Proposition 4.3 and Hölder's inequality in the last line.

In the next step we shall consider the time direction. In contrast to the argument for the tangential space directions we cannot differentiate the parabolic system with respect to $t$. Instead, we will bypass this lack of differentiability by the use of second-order finite time differences of $u$. For this we first fix $0<|h| \leqslant \frac{1}{16^{2}} \min \left\{R^{2}-\varrho^{2}, \varrho^{2}\right\}$ and $\sigma_{2} \in\left(2, \min \left\{\sigma_{1}, 2+\frac{4}{n}\right\}\right]$ to be the higher integrability exponent from Lemma 4.12 (in the case $\tilde{\sigma}_{2}<\sigma_{2}$ we take the smaller value $\tilde{\sigma}_{2}$ instead). Then, we consider $z_{0} \in Q_{\varrho / 2}^{+} \cup \Gamma_{\varrho / 2}$. Combining Lemmas 4.14 and 4.12 we infer for $\sigma \in\left(2, \sigma_{2}\right], 0<s \leqslant \varrho / 2$ and $0<\varepsilon \leqslant 1$ that

$$
\begin{aligned}
\int_{Q_{s / 2}^{+}} \frac{\left|\tau_{-h} \tau_{h} u\right|^{\sigma}}{|h|^{\frac{\sigma}{2}}} d z \leqslant & c \int_{Q_{s}^{+}}\left|\tau_{h} D u\right|^{\sigma}+\left|\tau_{-h} D u\right|^{\sigma} d z+c\left|Q_{s}^{+}\right|^{1-\frac{\sigma}{2}}\left(\int_{Q_{s}^{+}} \frac{\left|\tau_{-h} \tau_{h} u\right|^{2}}{|h|} d z\right)^{\frac{\sigma}{2}} \\
& +c|h|^{\frac{\sigma}{2}} \int_{Q_{s}^{+}} \gamma^{\sigma}\left(1+|D u|^{\sigma}\right)+|\mathcal{G}|^{\sigma} d z \\
\leqslant & \varepsilon^{\frac{\sigma}{2}} \int_{Q_{2 s}^{+}} \frac{\left|\tau_{-h} \tau_{h} u\right|^{\sigma}}{|h|^{\frac{\sigma}{2}}} d z+c\left|Q_{s}^{+}\right|^{1-\frac{\sigma}{2}}\left(\int_{Q_{2 s}^{+}} \frac{\left|\tau_{-h} \tau_{h} u\right|^{2}}{|h|}+\left|\tau_{h} D u\right|^{2}+\left|\tau_{-h} D u\right|^{2} d z\right)^{\frac{\sigma}{2}} \\
& +c|h|^{\frac{\sigma}{2}} \int_{Q_{2 s}^{+}} \gamma^{\sigma}\left(1+|D u|^{\sigma}\right)+\varepsilon^{-\frac{\sigma}{2}}|\mathcal{G}|^{\sigma} d z
\end{aligned}
$$

where we have used the abbreviation $\mathcal{G}(x, t):=|G(x, t)|+\left|\tau_{h} G(x, t)\right|+\left|\tau_{-h} G(x, t)\right|$ and $c$ depends on $n, N$ and $L / \nu$ only. Now, we consider two radii $r_{1}, r_{2}$ such that $\varrho / 4 \leqslant r_{1}<r_{2} \leqslant \varrho / 2$. Then, we cover $Q_{r_{1}}^{+}$by a finitely many parabolic cylinders $\left\{Q_{s / 2}^{+}\left(z_{i}\right)\right\}_{i=1}^{\mathfrak{B}}$ of radius $s=\left(r_{2}-r_{1}\right) / 4$ intersecting at most $c(n)$ times and such that $Q_{2 s}^{+}\left(z_{i}\right) \subset$ $Q_{r_{2}}^{+}$for $i=1, \ldots, \mathfrak{B}$. Applying the preceding estimate on each of the cylinders $Q_{s / 2}^{+}\left(z_{i}\right)$, summing up the resulting inequalities over $i=1, \ldots, \mathfrak{B}$, and finally choosing $\varepsilon$ small enough (i.e. $c(n) \varepsilon=\frac{1}{2}$ ) yields

$$
\begin{aligned}
\int_{Q_{r_{1}}^{+}} \frac{\left|\tau_{-h} \tau_{h} u\right|^{\sigma}}{|h|^{\frac{\sigma}{2}}} d z \leqslant & \frac{1}{2} \int_{Q_{r_{2}}^{+}} \frac{\left|\tau_{-h} \tau_{h} u\right|^{\sigma}}{|h|^{\frac{\sigma}{2}}} d z+c\left(r_{2}-r_{1}\right)^{\left(1-\frac{\sigma}{2}\right)(n+2)}\left(\int_{Q_{\varrho / 2}^{+}} \frac{\left|\tau_{-h} \tau_{h} u\right|^{2}}{|h|}+\left|\tau_{h} D u\right|^{2}+\left|\tau_{-h} D u\right|^{2} d z\right)^{\frac{\sigma}{2}} \\
& +c|h|^{\frac{\sigma}{2}} \int_{Q_{\varrho}^{+}} \gamma^{\sigma}\left(1+|D u|^{\sigma}\right)+|G|^{\sigma} d z,
\end{aligned}
$$

where $c=c(n, N, L / v)$. Here we have also used the fact $|h| \leqslant(\varrho / 16)^{2}$ in order to have $\int_{Q_{\varrho / 2}^{+}} f(x, t \pm h) d z \leqslant$ $\int_{Q_{\varrho}^{+}} f d z$ for an integrable function $f \geqslant 0$. Now, we apply the iteration lemma [7, Lemma 2.4] to absorb the first integral appearing on the right-hand side on the left one. This yields

$$
\begin{aligned}
\int_{Q_{\varrho / 4}^{+}\left(z_{0}\right)} \frac{\left|\tau_{-h} \tau_{h} u\right|^{\sigma}}{|h|^{\frac{\sigma}{2}}} d z \leqslant & c\left(\int_{Q_{\varrho / 2}^{+}} \frac{\left|\tau_{-h} \tau_{h} u\right|^{2}}{|h|}+\left|\tau_{h} D u\right|^{2}+\left|\tau_{-h} D u\right|^{2} d z\right)^{\frac{\sigma}{2}} \\
& +c|h|^{\frac{\sigma}{2}} \int_{Q_{\varrho}^{+}} \gamma^{\sigma}\left(1+|D u|^{\sigma}\right)+|G|^{\sigma} d z,
\end{aligned}
$$


where $c=c(n, N, L / v)$. The right-hand side of the preceding inequality, i.e. the first integral, can be further estimated by (4.13) and Corollary 4.9 (with $h$ and $-h$ ) and Hölder's inequality to yield for any choice of $0<|h| \leqslant(\varrho / 16)^{2}$ that $\int_{Q_{\varrho / 4}^{+}}\left|\tau_{-h} \tau_{h} u\right|^{\sigma} d z \leqslant c|h|^{\sigma} M^{\sigma}$ where

$$
M^{\sigma}:=\left(\gamma^{\sigma}+\varrho^{-\sigma}\right) \int_{Q_{\varrho}^{+}} 1+|D u|^{\sigma} d z+\|G\|_{\mathcal{N}^{0, \vartheta ; \sigma}\left(Q_{\varrho}^{+}\right)}^{\sigma}
$$

Noting that $\tau_{-h} \tau_{h} u(t)=\tau_{h} \tau_{h} u(t-h)$ as well as $|h| \leqslant(\varrho / 16)^{2}$ the preceding estimate can be turned into

$$
\int_{Q_{\varrho / 8}^{+}}\left|\tau_{h} \tau_{h} u\right|^{\sigma} d z \leqslant c|h|^{\sigma} M^{\sigma}
$$

Therefore we are in a position to apply Lemma 2.4 with $\left(\alpha, \beta, M, h_{0}\right)$ replaced by $\left(1,1-\vartheta / 4, M,(\varrho / 16)^{2}\right)$ and Poincaré's inequality to infer

$$
\int_{Q_{\varrho / 8}^{+}}\left|\tau_{h} u\right|^{\sigma} d z \leqslant c|h|^{\sigma\left(1-\frac{\vartheta}{4}\right)}\left[\varrho^{\frac{\sigma \vartheta}{2}} M^{\sigma}+\varrho^{\sigma\left(\frac{\vartheta}{2}-2\right)} \int_{Q_{\varrho / 4}^{+}}|u|^{\sigma} d z\right] \leqslant c|h|^{\sigma\left(1-\frac{\vartheta}{4}\right)} \varrho^{\frac{\sigma \vartheta}{2}} M^{\sigma},
$$

for any choice of $0<|h| \leqslant(\varrho / 32)^{2}$. Note that $c=c(n, N, L / \nu, \vartheta, \sigma)$ and $c \rightarrow \infty$ when $\vartheta \downarrow 0$ or $\sigma \downarrow 2$.

In the next step we shall improve this estimate in the sense that we enlarge the exponent of $|h|$ on the right-hand side from $\sigma\left(1-\frac{\vartheta}{4}\right)$ to $\sigma$. For this we once again apply Lemma 4.14 and obtain

$$
\begin{aligned}
\int_{Q_{\varrho / 32}^{+}} \frac{\left|\tau_{-h} \tau_{h} u\right|^{\sigma}}{|h|^{\frac{\sigma}{2}}} d z \leqslant & c\left(f_{Q_{\varrho / 32}^{+}} \frac{\left|\tau_{-h} \tau_{h} u\right|^{2}}{|h|} d z\right)^{\frac{\sigma}{2}}+c f_{Q_{\varrho / 16}^{+}}\left|\tau_{h} D u\right|^{\sigma}+\left|\tau_{-h} D u\right|^{\sigma} d z \\
& +c|h|^{\frac{\sigma}{2}(1+\vartheta)} f_{Q_{\varrho / 16}^{+}} \gamma^{\sigma}\left(1+|D u|^{\sigma}\right)+|h|^{\frac{-\vartheta \sigma}{2}}\left(\left|\tau_{h} G\right|^{\sigma}+\left|\tau_{-h} G\right|^{2}\right) d z .
\end{aligned}
$$

To estimate the second integral on the right-hand side we apply in turn Corollaries 4.13 and 4.6 (for $h$ and $-h$ and with $\left.\varepsilon=h^{1-\vartheta} \varrho^{2 \vartheta}\right)$, Hölder's inequality and $|h| \leqslant \varrho^{2}$ to infer for any $\sigma \in\left(2, \sigma_{2}\right]$ that

$$
\begin{aligned}
\int_{Q_{\varrho / 16}^{+}}\left|\tau_{ \pm h} D u\right|^{\sigma} d z \leqslant & c\left(f_{Q_{\varrho / 8}^{+}(z 0)}\left|\tau_{ \pm h} D u\right|^{2} d z\right)^{\frac{\sigma}{2}}+c|h|^{\frac{\sigma}{2}(\vartheta-1)} \varrho^{-\sigma \vartheta} f_{Q_{\varrho / 8}^{+}}\left|\tau_{ \pm h} u\right|^{\sigma} d z \\
& +c|h|^{\frac{\sigma}{2}(1+\vartheta)}\left[\gamma^{\sigma} f_{Q_{\varrho / 8}^{+}}\left(1+|D u|^{\sigma}\right) d z+\frac{\varrho^{\sigma \vartheta}}{|h|^{\sigma \vartheta}} f_{Q_{\varrho / 8}^{+}}\left|\tau_{ \pm h} G\right|^{\sigma} d z\right] \\
\leqslant & c|h|^{\frac{\sigma}{2}(\vartheta-1)} \varrho^{-\sigma \vartheta} \int_{Q_{\varrho / 8}^{+}}\left|\tau_{ \pm h} u\right|^{\sigma} d z \\
& +c|h|^{\frac{\sigma}{2}(1+\vartheta)}\left[\gamma^{\sigma} f_{Q_{\varrho / 8}^{+}}\left(1+|D u|^{\sigma}\right) d z+\frac{\varrho^{\sigma \vartheta}}{|h|^{\sigma \vartheta}} \underset{Q_{\varrho / 8}^{+}}{f}\left|\tau_{ \pm h} G\right|^{\sigma} d z\right],
\end{aligned}
$$

where $c=c(n, N, L / v, \vartheta, \sigma)$, and multiplying both sides of the preceding inequality $\left|Q_{\varrho / 16}^{+}\right|$yields the estimate without mean values. We use this inequality together with (4.26) (for $\pm h$ ) and the assumption $G \in \mathcal{N}^{0, \vartheta ; \sigma}\left(Q_{\varrho}^{+} ; \mathbb{R}^{N}\right)$ to get

$$
\int_{Q_{\varrho}^{+} / 16}\left|\tau_{ \pm h} D u\right|^{\sigma} d z \leqslant c|h|^{\frac{\sigma}{2}+\frac{\vartheta \sigma}{4}} \varrho^{-\frac{\sigma \vartheta}{2}} M^{\sigma}+c|h|^{\frac{\sigma}{2}(1+\vartheta)}\left[M^{\sigma}+\varrho^{\sigma \vartheta}\|G\|_{\mathcal{N}^{0, \vartheta ; \sigma}\left(Q_{\varrho}^{+} ; \mathbb{R}^{N}\right)}^{\sigma}\right] \leqslant c|h|^{\frac{\sigma}{2}+\frac{\vartheta \sigma}{4}} \varrho^{-\frac{\sigma \vartheta}{2}} M^{\sigma} .
$$


We insert this into (4.27) to bound the second integral on the right-hand side there and then use (4.13) to estimate the first one. Finally, we use the hypothesis $G \in \mathcal{N}^{0, \vartheta ; \sigma}\left(Q_{\varrho}^{+} ; \mathbb{R}^{N}\right)$ to infer

$$
\int_{Q_{\varrho / 32}^{+}}\left|\tau_{-h} \tau_{h} u\right|^{\sigma} d z \leqslant c|h|^{\sigma}\left[\left(|h|^{\frac{\vartheta \sigma}{2}}+h^{\frac{\vartheta \sigma}{4}} \varrho^{-\frac{\sigma \vartheta}{2}}\right) M^{\sigma}+|h|^{\vartheta \sigma}\|G\|_{\mathcal{N}^{0, \vartheta ; \sigma}\left(Q_{\varrho}^{+} ; \mathbb{R}^{N}\right)}^{\sigma}\right] \leqslant c|h|^{\sigma+\frac{\vartheta \sigma}{4}} \varrho^{-\frac{\sigma \vartheta}{2}} M^{\sigma},
$$

where $c=c(n, N, L / v, \vartheta, \sigma)$. Using again $\tau_{-h} \tau_{h} u(t)=\tau_{h} \tau_{h} u(t-h)$ we arrive at

$$
\int_{Q_{\varrho / 64}^{+}}\left|\tau_{h} \tau_{h} u\right|^{\sigma} d z \leqslant \int_{Q_{\varrho / 32}^{+}}\left|\tau_{-h} \tau_{h} u\right|^{\sigma} d z \leqslant c|h|^{\sigma+\frac{\vartheta \sigma}{4}} \varrho^{-\frac{\sigma \vartheta}{2}} M^{\sigma},
$$

for any $0<|h| \leqslant(\varrho / 64)^{2}$. Having arrived at this stage we are in the position to apply again Lemma 2.4 now with $\left(1+\frac{\vartheta}{4}, 1, c \varrho^{-\frac{\vartheta}{2}} M, \frac{1}{2}(\varrho / 16)^{2}\right)$ instead of $\left(\alpha, \beta, M, h_{0}\right)$ to infer

$$
\int_{Q_{\varrho / 64}^{+}}\left|\tau_{h} u\right|^{\sigma} d z \leqslant c|h|^{\sigma}\left(M^{\sigma}+\varrho^{-2 \sigma} \int_{Q_{\varrho}^{+}}|u|^{\sigma} d z\right) \leqslant c|h|^{\sigma} M^{\sigma},
$$

for any $0<|h| \leqslant(\varrho / 128)^{2}$. Note that we have also applied Poincaré's inequality slice wise in the last line. We note that $c=c(n, N, L / v, \vartheta, \sigma)$. By the standard estimate for difference quotients this implies $\partial_{t} u \in L^{\sigma}\left(Q_{R / 64}^{+} ; \mathbb{R}^{N}\right)$. Moreover, the following estimate holds

$$
\int_{Q_{\varrho / 64}^{+}}\left|\partial_{t} u\right|^{\sigma} d z \leqslant c M^{\sigma} .
$$

Finally, a different choice of the radii allows us to show that the preceding estimate holds with $Q_{\varrho / 2}^{+}$as domain of integration instead of $Q_{\varrho / 64}^{+}$. This proves the assertion of the lemma concerning the $L^{\sigma}$-estimate of $\partial_{t} u$.

To obtain the desired estimate for the normal derivative $D_{n} D u$, we argue similarly to the proof of Proposition 4.10. To be precise, we first attain the analogue of (4.17) for the $L^{\sigma}$-norm of $\tau_{h}^{n}[D u]$ instead of the $L^{2}$-norm. Then using the $L^{\sigma}$-estimate for $\left|D_{\alpha} D u\right|$ and $\left|\partial_{t} u\right|$ from above we arrive at

$$
\int_{Q_{\varrho / 2}^{+}}\left|\tau_{h}^{n}[D u]\right|^{\sigma} d z \leqslant c h^{\sigma}\left[\left(\varrho^{-\sigma}+\gamma^{\sigma}\right) \int_{Q_{\varrho}^{+}}\left(1+|D u|^{\sigma}\right) d z+\|G\|_{\mathcal{N}^{0, v ; \sigma}\left(Q_{\varrho}^{+} ; \mathbb{R}^{N}\right)}\right],
$$

for any choice of $0<h \leqslant \varrho / 2$. Therefore, we conclude that $D_{n} D u \in L^{\sigma}\left(Q_{R / 2}^{+} ; \mathbb{R}^{N}\right)$ for any $\sigma \in\left(2, \sigma_{2}\right]$. Furthermore, we have

$$
\int_{Q_{\varrho / 2}^{+}}\left|D_{n} D u\right|^{\sigma} d z \leqslant c\left[\left(\varrho^{-\sigma}+\gamma^{\sigma}\right) \int_{Q_{\varrho}^{+}}\left(1+|D u|^{\sigma}\right) d z+\|G\|_{\mathcal{N}^{0, v ; \sigma}\left(Q_{\varrho}^{+} ; \mathbb{R}^{N}\right)}\right],
$$

for any $0<\varrho \leqslant R / 2$. This proves the asserted $L^{\sigma}$-estimate for $D_{n} D u$ which together with (4.25) yields the higher integrability of $D^{2} u$. This completes the proof of the lemma.

At the end of this section we provide an improved (compared to Corollary 4.9) estimate for finite differences in time. It will be applied later in the proof of the dimension reduction for the singular set of solutions of certain regularized systems.

Corollary 4.16. Let $u$ be a weak solution of (4.1) on $Q_{R}^{+}$according to Definition 4.1 and suppose that $G \in$ $\mathcal{N}^{0, \vartheta, \sigma_{1}}\left(Q_{R}^{+} ; \mathbb{R}^{N}\right)$ for some $\sigma_{1}>2$. Then there exists $\sigma_{2}=\sigma_{2}(n, L / v) \in\left(2, \min \left\{\sigma_{1}, 2+\frac{4}{n}\right\}\right]$ such that for all $\sigma \in\left(2, \sigma_{2}\right], 0<\varrho \leqslant R$ and $0<|h| \leqslant(\varrho / 2)^{2}$ there holds, for a constant $c=c(n, N, L / v)$, that

$$
f_{Q_{\varrho / 2}^{+}}\left|\tau_{h} D u\right|^{\sigma} d z \leqslant c|h|^{\frac{\sigma}{2}}\left[\left(\gamma^{\sigma}+\varrho^{-\sigma}\right) \int_{Q_{\varrho}^{+}}\left(1+|D u|^{\sigma}\right) d z+\|G\|_{\mathcal{N}^{0, \xi ; \sigma}\left(Q_{\varrho}^{+}\right)}^{\sigma}\right] \text {. }
$$


Proof. Let $\sigma_{2} \in\left(2, \min \left\{\sigma_{1}, 2+\frac{4}{n}\right\}\right]$ be the minimum of the corresponding constants of Lemma 4.12 and Proposition 4.15. Applying Lemma 4.12 with the choice $\varepsilon=1$ on the cylinders $Q_{\varrho / 2}^{+}, Q_{3 \varrho / 4}^{+}$instead of $Q_{\varrho / 2}^{+}, Q_{\varrho}^{+}$and noting that $\left|\tau_{-h} \tau_{h} u(t)\right| \leqslant\left|\tau_{h} u(t)\right|+\left|\tau_{h} u(t-h)\right|$ we infer for $0<|h| \leqslant(\varrho / 4)^{2}$ (then $\left.(3 \varrho / 4)^{2}+|h| \leqslant \varrho^{2}\right)$ that

$$
\int_{Q_{\varrho / 2}^{+}}\left|\tau_{h} D u\right|^{\sigma} d z \leqslant c\left(\int_{Q_{\varrho}^{+}}\left|\tau_{h} D u\right|^{2} d z\right)^{\frac{\sigma}{2}}+c|h|^{-\frac{\sigma}{2}} \int_{Q_{\varrho}^{+}}\left|\tau_{h} u\right|^{\sigma} d z+c|h|^{\frac{\sigma}{2}} \int_{Q_{\varrho}^{+}} \gamma^{\sigma}\left(1+|D u|^{\sigma}\right)+|G|^{\sigma} d z .
$$

In the preceding inequality we use Corollary 4.9 to bound the first integral of the right-hand side, while for the second one we recall that $u$ is weakly differentiable with respect to time fulfilling the $L^{\sigma}$-estimate for $\partial_{t} u$ from Proposition 4.15. This leads us to

$$
\int_{Q_{\varrho / 2}^{+}}\left|\tau_{h} D u\right|^{\sigma} d z \leqslant c|h|^{\frac{\sigma}{2}}\left[\left(\gamma^{\sigma}+\varrho^{-\sigma}\right) \int_{Q_{2 \varrho}^{+}}\left(1+|D u|^{\sigma}\right) d z+\|G\|_{\mathcal{N}^{0, \xi ; \sigma}\left(Q_{2 \varrho}^{+}\right)}^{\sigma}\right],
$$

for any $\sigma \in\left(2, \sigma_{2}\right]$. By a different choice of radii we infer the asserted estimate.

\subsection{The initial boundary}

Now, we turn our attention to the initial boundary, where we again consider the parabolic system (4.1), but now on the cylinder $Q=Q_{R}^{0}$. Note that in the situation of initial boundary we can renounce the regularity assumption in time (4.2) $)_{4}$ on the coefficients $a$. As in the lateral boundary situation we will prove suitable $L^{2}$-estimates, respectively $L^{\sigma}$-estimates, for $D^{2} u$ and $\tau_{h} D u$ for some $\sigma>2$. We mention that the initial boundary situation is much easier, since we can treat all second space derivatives in the usual way. Afterwards there is only one derivative missing, namely the first time derivative for which we can exploit the system. Throughout the section we consider weak solutions under the following assumptions:

Definition 4.17. By a weak solution of the parabolic initial problem (4.1) on $Q_{R}^{0}$ we mean a function $u \in$ $L^{2}\left(\Lambda_{R^{2}}^{0} ; W^{1,2}\left(B_{R} ; \mathbb{R}^{N}\right)\right)$ with $u(\cdot, 0)=0$ on $B_{R}$ which satisfies (4.1) in the weak sense. Moreover, the structure conditions $(4.2)_{1}-(4.2)_{3}$ are in force and $G=0$.

For weak solutions in the sense of the preceding definition we shall show the existence of second-order spatial derivatives and the time derivative together with their higher integrability.

Proposition 4.18. Let $u$ be a weak solution of (4.1) on $Q_{R}^{0}$ according to Definition 4.17. Then, for any $0<r<R$ we have $D u \in L^{2+\frac{4}{n}}\left(Q_{r}^{0}, \mathbb{R}^{N n}\right)$ and there exists $\sigma_{1}=\sigma_{1}(n, N, L / v) \in\left(2,2+\frac{4}{n}\right]$ such that $D^{2} u \in L^{\sigma_{1}}\left(Q_{r}^{0}, \mathbb{R}^{N n^{2}}\right)$ and $\partial_{t} u \in L^{\sigma_{1}}\left(Q_{r}^{0}, \mathbb{R}^{N}\right)$. Moreover, for any $0<\varrho \leqslant R$ and $\sigma \in\left[2, \sigma_{1}\right]$, and for a constant $c=c(n, N, L / v)$ there holds

$$
\int_{Q_{\varrho / 2}^{0}}\left|D^{2} u\right|^{\sigma}+\left|\partial_{t} u\right|^{\sigma} d z \leqslant c\left(\gamma^{\sigma}+\varrho^{-\sigma}\right) \int_{Q_{\varrho}^{0}}\left(1+|D u|^{\sigma}\right) d z .
$$

Proof. First, we show that the second space derivatives $D^{2} u$ exist in $L^{2}$ up to the initial boundary. Here, we proceed along the lines of the lateral boundary situation when treating the tangential derivatives in Proposition 4.3 apart from the difference that we can take any spatial direction $\alpha=1, \ldots, n$. Since the proof is very much similar to the one of Proposition 4.3 we only outline the adjustments to be made in the initial boundary situation. As in the proof of Proposition 4.3 we start with the Steklov-formulation of the system, now on $B_{\varrho} \times(0, t)$ for some $t \in\left(0, \varrho^{2}\right)$. Then, we choose the test-function $\varphi_{\varepsilon}=\eta^{2} \zeta_{\varepsilon} \tau_{h}^{\alpha} u_{\lambda}$, where the cut-off function $\eta \in C_{0}^{\infty}\left(B_{\varrho}\right)$ is chosen as in the proof of Proposition 4.3 , and $\zeta_{\varepsilon} \in W^{1, \infty}(\mathbb{R})$ is defined to be $\zeta_{\varepsilon}=0$ on $(-\infty, 0], \zeta_{\varepsilon} \equiv 1$ on $[\varepsilon, \infty)$ and $\zeta_{\varepsilon}(s)=s / \varepsilon$ on $(0, \varepsilon)$ with $\varepsilon \in\left(0, \varrho^{2}\right)$. When testing the parabolic system with $\varphi_{\varepsilon}$, the interesting term is the one involving the time derivative (i.e. the analog to (4.4)) since this is the only one where the initial condition comes into the play. Similarly to (4.4) we first rewrite 


$$
\int_{0}^{t} \int_{B_{R}} \partial_{t} \tau_{h}^{\alpha}\left[u_{\lambda}\right] \cdot \varphi_{\varepsilon} d x d \tau=\frac{1}{2} \int_{B_{\varrho}}\left|\tau_{h}^{\alpha} u_{\lambda}(\cdot, t)\right|^{2} \eta^{2} \zeta_{\varepsilon}(t) d x-\frac{1}{2} \int_{0}^{t} \int_{B_{\varrho}}\left|\tau_{h}^{\alpha} u_{\lambda}\right|^{2} \eta^{2} \partial_{t} \zeta_{\varepsilon} d x d \tau
$$

and then estimate the second term on the right-hand side by

$$
\frac{1}{2} \int_{0}^{t} \int_{B_{Q}}\left|\tau_{h}^{\alpha} u_{\lambda}\right|^{2} \eta^{2} \partial_{t} \zeta_{\varepsilon} d x d \tau \leqslant \frac{1}{2 \varepsilon} \int_{0}^{\varepsilon} \int_{B_{\varrho}}\left|\tau_{h}^{\alpha} u_{\lambda}\right|^{2} \eta^{2} d x d \tau
$$

Then, passing to the limits $\lambda \downarrow 0$ and $\varepsilon \downarrow 0$ we find, due to our initial condition imposed on $u$, that the right-hand side converges to zero. Therefore, starting from the Steklov-formulation as mentioned above, passing limits $\lambda \downarrow 0$ and $\varepsilon \downarrow 0$ we arrive at

$$
\begin{aligned}
& \frac{1}{2} \int_{B_{Q}}\left|\tau_{h}^{\alpha} u(\cdot, t)\right|^{2} \eta^{2} d x+\int_{0}^{t} \int_{B_{Q}}\left\langle\tau_{h}^{\alpha}[a(\cdot, D u)], D \tau_{h}^{\alpha} u\right\rangle \eta^{2} d x d \tau \\
& \quad=\int_{0}^{t} \int_{B_{\varrho}} 2\left\langle\tau_{h}^{\alpha}[a(\cdot, D u)], D \eta \otimes \tau_{h}^{\alpha} u\right\rangle \eta d x d \tau,
\end{aligned}
$$

for a.e. $t \in\left(0, \varrho^{2}\right)$. At this stage we can proceed completely similar to the proof of Proposition 4.3. This leads us to the following Caccioppoli-type estimate:

$$
\sup _{t \in\left(0, \varrho^{2}\right)} \int_{B_{\varrho / 2}}\left|D_{\alpha} u(\cdot, t)\right|^{2} d x+\int_{Q_{\varrho / 2}^{0}}\left|D_{\alpha} D u\right|^{2} d z \leqslant c\left(\gamma^{2}+\varrho^{-2}\right) \int_{Q_{\varrho}^{0}}\left(1+|D u|^{2}\right) d z,
$$

valid for any $\alpha=1, \ldots, n$ and for a constant $c=c(n, N, L / v)$. With the arguments of the proof of Lemma 4.11 this immediately implies the first assertion of the lemma, i.e. that $D u \in L^{2+\frac{4}{n}}\left(Q_{\varrho / 4}^{0}, \mathbb{R}^{N n}\right)$ (note that we always can replace the radius $\varrho / 4$ by some arbitrary radius $<R$ ) together with the estimate

$$
\int_{Q_{\varrho / 4}^{0}}|D u|^{2+\frac{4}{n}} d z \leqslant c\left[\left(\gamma^{2}+\varrho^{-2}\right) \int_{Q_{\varrho}^{0}}\left(1+|D u|^{2}\right) d z\right]^{1+\frac{2}{n}},
$$

where $c=c(n, N, L / v)$.

Now, it remains to prove the higher integrability of $D^{2} u$ up to the initial boundary. For this we exploit the fact that $D_{\alpha} u, \alpha=1, \ldots, n$, is a weak solution of the following linear parabolic system (see the proof of Proposition 4.15)

$$
\int_{Q_{R}^{0}} D_{\alpha} u \cdot \varphi_{t}-\left\langle\partial_{w} a(\cdot, D u) D D_{\alpha} u, D \varphi\right\rangle d z=\int_{Q_{R}^{0}}\left\langle\partial_{x_{\alpha}} a(\cdot, D u), D \varphi\right\rangle d z,
$$

for all $\varphi \in C_{0}^{\infty}\left(Q_{R}^{0}, \mathbb{R}^{N}\right)$. Moreover, we have $D_{\alpha} u(\cdot, 0)=0$. This can for instance be seen as a consequence of the Caccioppoli inequality for $u$ on cylinders of the type $B_{r} \times(0, \lambda)$ with $\lambda \in\left(0, R^{2}\right)$. For the sake of brevity we only sketch the argument. To achieve such a Caccioppoli inequality we proceed as in the proof of the Caccioppoli inequality for $\tau_{h}^{\alpha} u$ from above - but without taking the finite differences. After testing the system with $\varphi_{\varepsilon}=\eta^{2} \zeta_{\varepsilon} u_{\lambda}$ (with a cutoff function $\eta \in C_{0}^{\infty}\left(B_{R}\right)$ and $\zeta_{\varepsilon}$ as above) and performing the standard computations we arrive at the following analogue of (4.28):

$$
\frac{1}{2} \int_{B_{R}}|u(\cdot, t)|^{2} \eta^{2} d x+\int_{0}^{\lambda} \int_{B_{R}}\langle a(\cdot, D u), D u\rangle \eta^{2} d x d \tau=\int_{0}^{\lambda} \int_{B_{R}} 2\langle a(\cdot, D u), D \eta \otimes u| \eta d x d \tau .
$$


The first integral on the left-hand side is non-negative, while for the second one we can use the ellipticity $(1.2)_{2}$ of the vector field $a$, and for the right-hand side we can use the growth assumption (1.2) $)_{1}$. This leads us to

$$
v \int_{0}^{\lambda} \int_{B_{R}}|D u|^{2} \eta^{2} d x d \tau \leqslant 2 L \int_{0}^{\lambda} \int_{B_{R}}(1+|D u|) \eta|u||D \eta| d x d \tau .
$$

Now, we choose $\eta$ such that $\eta \equiv 1$ on $B_{r}, 0<r<R, 0 \leqslant \eta \leqslant 1$ and $|D \eta| \leqslant c /(R-r)$. Then, applying Young's inequality and absorbing $|D u|^{2}$ in the standard way we arrive at

$$
\int_{0}^{\lambda} \int_{B_{r}}|D u|^{2} d x d \tau \leqslant c(L / \nu) \int_{0}^{\lambda} \int_{B_{R}} \frac{|u|^{2}}{(R-r)^{2}}+\frac{|u|}{(R-r)} d x d \tau .
$$

This implies by Hölder's inequality and due to our initial condition on $u$ that

$$
\lim _{\lambda \downarrow 0} \frac{1}{\lambda} \int_{0}^{\lambda} \int_{B_{r}}|D u|^{2} d z \leqslant \frac{c}{(R-r)^{2}} \lim _{\lambda \downarrow 0} \frac{1}{\lambda} \int_{0}^{\lambda} \int_{B_{R}}|u|^{2} d z+\frac{c}{(R-r)} \lim _{\lambda \downarrow 0}\left(\frac{1}{\lambda} \int_{0}^{\lambda} \int_{B_{R}}|u|^{2} d z\right)^{\frac{1}{2}}=0,
$$

proving the assertion $D u(\cdot, 0)=0$ on $B_{r}$ for any $0<r<R$.

Now, we come back to the proof of the higher integrability of $D_{\alpha} D u$. From our growth and ellipticity assumptions (4.2) $)_{1}$ and (4.2) 2 we know that $\left\langle\partial_{w} a(x, t, w) \tilde{w}, \tilde{w}\right\rangle \geqslant v|\tilde{w}|^{2}$ and $\left|\partial_{w} a(x, t, w)\right| \leqslant L$ for all $w, \tilde{w} \in \mathbb{R}^{N n}$. Moreover, from $(4.2)_{3}$ and the preceding estimate we infer that $\partial_{x_{\alpha}} a(x, t, D u) \in L^{2+\frac{n}{4}}\left(Q_{\varrho}^{0} ; \mathbb{R}^{N n}\right)$ (note here that $\left.\left|\partial_{x_{\alpha}} a(x, t, D u)\right| \leqslant L \gamma(1+|D u|)\right)$. Therefore, recalling that $D_{\alpha} u$ is a solution of (4.29) we can apply an up to the initial boundary higher integrability result (see for instance [31]) to infer the existence of $\sigma_{1}=\sigma_{1}(n, N, L / v) \in\left(2,2+\frac{4}{n}\right.$ ] such that $D D_{\alpha} u \in L^{\sigma_{1}}\left(Q_{\varrho / 2}^{0} ; \mathbb{R}^{N n}\right)$ and, moreover, for any $0<\varrho \leqslant R$ and $\sigma \in\left[2, \sigma_{1}\right]$ we have

$$
\begin{aligned}
f_{Q_{\varrho / 4}^{0}}\left|D_{\alpha} D u\right|^{\sigma} d z & \leqslant c\left(f_{Q_{\varrho / 2}^{0}}\left|D_{\alpha} D u\right|^{2} d z\right)^{\frac{\sigma}{2}}+c L^{\sigma} \gamma^{\sigma} f_{Q_{\varrho / 2}^{0}}(1+|D u|)^{\sigma} d z \\
& \leqslant c(n, N, L / \nu)\left(\gamma^{\sigma}+\varrho^{-\sigma}\right) f_{Q_{\varrho}^{+}}\left(1+|D u|^{\sigma}\right) d z .
\end{aligned}
$$

Summing up over $\alpha=1, \ldots, n$ leads to the result with radii $\varrho / 4, \varrho$ instead of $\varrho / 2, \varrho$. However, by a different choice of the radii we obtain the estimate for $D^{2} u$. Now, we can utilize the parabolic system in the usual way to infer the existence of $\partial_{t} u$ in $L^{\sigma_{1}}$ together with the asserted estimate. Since this is standard we omit the details and finish the proof of the lemma.

In the next two lemmas we are going to prove estimates for the finite differences in time of $D u$. Roughly speaking, this means that $D u$ admits a half time-derivative in $L^{2}$, respectively in $L^{\sigma}$ for some $\sigma>2$.

Lemma 4.19. Let $u$ be a weak solution of (4.1) on $Q_{R}^{0}$ according to Definition 4.17. Then, for any $z_{0}=\left(x_{0}, t_{0}\right) \in$ $Q_{R}^{0} \cup D_{R}$ and $0<\varrho \leqslant R$ and $0<h \leqslant(\varrho / 2)^{2}$ such that $Q_{\varrho}^{0} \subset Q_{R}^{0}$ there holds

$$
\int_{Q_{\varrho / 2}^{0}\left(z_{0}\right)}\left|\tau_{h} D u\right|^{2} d z \leqslant c(L) h \int_{Q_{\varrho}^{0}\left(z_{0}\right)} \gamma^{2}\left(1+|D u|^{2}\right)+\left|D^{2} u\right|^{2} d z .
$$

Proof. Without loss of generality we assume that $x_{0}=0$. We recall from the proof of Proposition 4.18 that $D_{\alpha} u$, $\alpha=1, \ldots, n$, is a weak solution of the linear parabolic system (4.29) with $D_{\alpha} u(\cdot, 0)=0$ on $B_{r}$ for any $0<r<R$. We choose a cut-off function $\eta \in C_{0}^{\infty}\left(B_{\varrho}\right)$ with $0 \leqslant \eta \leqslant 1, \eta=1$ on $B_{\varrho / 2}$ and $|D \eta| \leqslant c / \varrho$. Taking the test-function $\varphi(x, t)=\eta(x)^{2} \tau_{h} D_{\alpha} u(x, t)$ in the Steklov-formulation of (4.29), integrating with respect to $t$ over $\Lambda_{(\varrho / 2)^{2}}^{0}\left(t_{0}\right)$ and recalling the identity $\tau_{h} D_{\alpha} u=h \partial_{t}\left[D_{\alpha} u\right]_{h}$ we find 


$$
\begin{aligned}
\iint_{\Lambda_{(\varrho / 2)^{2}}^{0}\left(t_{0}\right)} \frac{\left|\tau_{h} D_{\alpha} u\right|^{2}}{h} \eta^{2} d x d t & =\iint_{\Lambda_{(\varrho / 2)^{2}}^{0}\left(t_{0}\right)} \int_{B_{Q}} \partial_{t}\left[D_{\alpha} u\right]_{h} \cdot \eta^{2} \tau_{h} D_{\alpha} u d x d t \\
& =\int_{\Lambda_{(\varrho / 2)^{2}}^{0}} \int_{\left.t_{0}\right)}\left\langle-\left[\partial_{w} a(\cdot, D u) D D_{\alpha} u\right]_{h}+\left[\partial_{x_{\alpha}} a(\cdot, D u)\right]_{h}, D\left(\eta^{2} \tau_{h} D_{\alpha} u\right)\right\rangle d x d t .
\end{aligned}
$$

By our assumptions $(4.2)_{1}$ and (4.2) 3 we have $\left|\partial_{w} a(\cdot, D u)\right| \leqslant L$ and $\left|\partial_{x_{\alpha}} a(\cdot, D u)\right| \leqslant L \gamma(1+|D u|)$. Together with Young's inequality, standard estimates for Steklov averages and the facts that $h \leqslant(\varrho / 2)^{2}$ and $|D \eta| \leqslant c / \varrho$ we therefore get

$$
\begin{aligned}
\iint_{(\varrho / 2)^{2}} \int_{\left.t_{0}\right)} \frac{\left|\tau_{h} D_{\alpha} u\right|^{2}}{h} \eta^{2} d x d t \leqslant & \frac{1}{2} \int_{\Lambda_{(\varrho / 2)^{2}}^{0}} \int_{\left.t_{0}\right)} \varrho_{B_{\varrho}} \varrho^{-2} \eta^{2}\left|\tau_{h} D_{\alpha} u\right|^{2}+\left|\tau_{h} D D_{\alpha} u\right|^{2} d x d t \\
& +c L^{2} \int_{Q_{\varrho}^{0}\left(t_{0}\right)} \gamma^{2}\left(1+|D u|^{2}\right)+\left|D D_{\alpha} u\right|^{2} d z .
\end{aligned}
$$

Since $\varrho^{-2}<h^{-1}$ by assumption we can absorb the first term of the right-hand side on the left. Moreover, we estimate $\left|\tau_{h} D D_{\alpha} u(x, t)\right| \leqslant\left|D D_{\alpha} u(x, t)\right|+\left|D D_{\alpha} u(x, t+h)\right|$ and utilize that $h<(\varrho / 2)^{2}$. Proceeding this way and summing up over $\alpha=1, \ldots, n$ we infer the asserted estimate.

Our next aim is to enlarge the exponents on both sides of the estimate for $\tau_{h} D u$ from the last lemma. The main strategy of the proof is quite similar to the one of Lemma 4.14 using the maximal and the sharp function.

Proposition 4.20. Let $u$ be a weak solution of (4.1) on $Q_{R}^{0}$ according to Definition 4.17 and $\sigma_{1}=\sigma_{1}(n, N, L / v) \in$ $\left(2,2+\frac{4}{n}\right]$ the constant from Proposition 4.18. Then, for any $\sigma \in\left[2, \sigma_{1}\right], 0<\varrho \leqslant R$ and $0<h \leqslant(\varrho / 2)^{2}$ there holds

$$
\int_{Q_{\varrho / 2}^{0}}\left|\tau_{h} D u\right|^{\sigma} d z \leqslant c(n, N, L / v) h^{\frac{\sigma}{2}}\left(\gamma^{\sigma}+\varrho^{-\sigma}\right) \int_{Q_{\varrho}^{0}}\left(1+|D u|^{\sigma}\right) d z .
$$

Proof. We consider a fixed parabolic cylinder of the type $Q_{r}\left(z_{1}\right) \subset Q_{\varrho / 2}^{0}, z_{1}=\left(x_{1}, t_{1}\right)$. In the sequel we distinguish the cases $0<h \leqslant(r / 2)^{2}$ and $h>(r / 2)^{2}$. In the case $h \leqslant(r / 2)^{2}$ we apply Lemma 4.19 to infer

$$
\int_{Q_{r}\left(z_{1}\right)}\left|\tau_{h} D u\right|^{2} d z \leqslant c(L) h \int_{Q_{2 r}^{0}\left(z_{1}\right)} \gamma^{2}\left(1+|D u|^{2}\right)+\left|D^{2} u\right|^{2} d z .
$$

Note that we applied here Lemma 4.19 in a situation where $Q_{r}^{0}\left(z_{1}\right)=Q_{r}\left(z_{1}\right)$. In the case $h>(r / 2)^{2}$ we choose a non-negative weight-function $\eta \in C_{0}^{\infty}\left(B_{r}\left(x_{1}\right)\right)$ with $f_{B_{r}\left(x_{1}\right)} \eta d x=1,0 \leqslant \eta \leqslant c$ and $|D \eta| \leqslant c / r$. Then, the weighted means $\left(\tau_{h} D u\right)_{\eta}(t)$ of $\tau_{h} D u$ are defined by

$$
\left(\tau_{h} D u\right)_{\eta}(t)=\int_{B_{r}\left(x_{1}\right)} \tau_{h} D u(\cdot, t) \eta d x .
$$

The mean square deviation of $\tau_{h} D u$ on $Q_{r}\left(z_{1}\right)$ from its mean value can then be decomposed as follows:

$$
\begin{aligned}
f_{\left.\Lambda_{r^{2}\left(t_{1}\right)}\right)} f_{B_{r}\left(x_{1}\right)}\left|\tau_{h} D u-\left(\tau_{h} D u\right)_{z_{1}, r}\right|^{2} d x d t \leqslant & 3\left[f_{\Lambda_{r^{2}}\left(t_{1}\right)} f_{B_{r}\left(x_{1}\right)}\left|\tau_{h} D u(x, t)-\left(\tau_{h} D u\right)_{\eta}(t)\right|^{2} d x d t\right. \\
& +f_{\Lambda_{r^{2}\left(t_{1}\right)}}\left|\left(\tau_{h} D u\right)_{\eta}(t)-f_{\Lambda_{r^{2}}\left(t_{1}\right)}\left(\tau_{h} D u\right)_{\eta}(\tau) d \tau\right|^{2} d t
\end{aligned}
$$




$$
\begin{aligned}
& \left.+\left|f_{\Lambda_{r^{2}}\left(t_{1}\right)}\left(\tau_{h} D u\right)_{\eta}(\tau) d \tau-\left(\tau_{h} D u\right)_{z_{1}, r}\right|^{2} d t\right] \\
= & : 3(I+I I+I I I),
\end{aligned}
$$

with the obvious meaning of $I-I I I$. To estimate $I$ we apply Poincaré's inequality slice wise for a.e. $t \in \Lambda_{r^{2}}\left(t_{1}\right)$ and use the fact that $r^{2} \leqslant 4 h$ to deduce

$$
I \leqslant c r^{2} f_{\Lambda_{r^{2}}\left(t_{1}\right)} f_{B_{r}\left(x_{1}\right)}\left|D\left(\tau_{h} D u\right)\right|^{2} d x d t \leqslant c h f_{Q_{r}\left(z_{1}\right)}\left|\tau_{h} D^{2} u\right|^{2} d z
$$

where $c=c(n)$. Here we have used once again (2.8). Similarly we obtain for III

$$
I I I \leqslant f_{\Lambda_{r^{2}}\left(t_{1}\right)} f_{B_{r}\left(x_{1}\right)}\left|\left(\tau_{h} D u\right)_{\eta}(\tau)-\tau_{h} D u(x, \tau)\right|^{2} d x d \tau=I \leqslant c(n) h \underset{Q_{r}\left(z_{1}\right)}{f}\left|\tau_{h} D^{2} u\right|^{2} d z .
$$

We now consider the integral $I I$ whose estimation is more involved. Here, for a fixed $i \in\{1, \ldots, N\}$ we define the test-function $\varphi: \mathbb{R}^{n+1} \rightarrow \mathbb{R}^{N}$ where $\varphi_{i}=\eta$ and $\varphi_{j}=0$ for $j \neq i$. We recall that $D_{\alpha} u, \alpha=1, \ldots, n$, is a weak solution of the linear parabolic system (4.29). Testing the Steklov formulation of (4.29) at levels $s$ and $s+h$ with the testfunction $\varphi$ and then taking the difference of the resulting equations yields, after integrating the difference with respect to $s$ over $(\tau, t)$, the following identity:

$$
\begin{aligned}
\left(\left[D_{\alpha} u_{i}\right]_{\lambda}\right)_{\eta}(t)-\left(\left[D_{\alpha} u_{i}\right]_{\lambda}\right)_{\eta}(\tau) & =\int_{\tau}^{t} \partial_{s}\left(\left[D_{\alpha} u_{i}\right]_{\lambda}\right)_{\eta} d s \\
& =\int_{\tau}^{t} f_{B_{r}\left(x_{1}\right)}\left\langle-\left[\left(\partial_{w} a(\cdot, D u) D D_{\alpha} u\right)_{i}\right]_{\lambda}+\left[\partial_{x_{\alpha}} a_{i}(\cdot, D u)\right]_{\lambda}, D \eta\right\rangle d x d s .
\end{aligned}
$$

We pass to the limit $\lambda \downarrow 0$ and recall that $\left|\partial_{w} a(\cdot, D u)\right| \leqslant L,\left|\partial_{x_{\alpha}} a(\cdot, D u)\right| \leqslant L \gamma(1+|D u|)$ and $|D \eta| \leqslant c / r$. Summing up over $i=1, \ldots, N$ leads us to

$$
\left|\left(D_{\alpha} u\right)_{\eta}(t)-\left(D_{\alpha} u\right)_{\eta}(\tau)\right| \leqslant c L r \int_{Q_{r}\left(z_{1}\right)}\left|D^{2} u\right|+\gamma(1+|D u|) d z .
$$

Using the preceding estimate with $(t, \tau)$, respectively $(t+h, \tau+h)$ and taking into account $r \leqslant 2 \sqrt{h}$ as well as $|f(t+h)| \leqslant\left|\tau_{h} f(t)\right|+|f(t)|$ we obtain for a.e. $t, \tau \in \Lambda_{r^{2}}\left(t_{1}\right)$ that

$$
\begin{aligned}
\left|\left(\tau_{h} D u\right)_{\eta}(t)-\left(\tau_{h} D u\right)_{\eta}(\tau)\right| & \leqslant\left|(D u)_{\eta}(t+h)-(D u)_{\eta}(\tau+h)\right|+\left|(D u)_{\eta}(t)-(D u)_{\eta}(\tau)\right| \\
& \leqslant c \sqrt{h} f_{Q_{r}\left(z_{1}\right)}\left|D^{2} u\right|+\left|\tau_{h} D^{2} u\right|+\gamma\left(1+|D u|+\left|\tau_{h} D u\right|^{2}\right) d z .
\end{aligned}
$$

We integrate this with respect to $t$ and $\tau$ over $\Lambda_{r^{2}}\left(t_{1}\right)$ and use Hölder's inequality to obtain

$$
I I \leqslant f_{\Lambda_{r^{2}}\left(t_{1}\right)} f_{\Lambda_{r^{2}}\left(t_{1}\right)}\left|\left(\tau_{h} D u\right)_{\eta}(t)-\left(\tau_{h} D u\right)_{\eta}(\tau)\right|^{2} d \tau d t \leqslant c f_{Q_{r}\left(z_{1}\right)}\left|w_{h}\right|^{2} d z,
$$

where $c=c(n, N, L)$ and

$$
w_{h}=\sqrt{h}\left(\left|D^{2} u\right|+\left|\tau_{h} D^{2} u\right|+\gamma\left(1+|D u|+\left|\tau_{h} D u\right|^{2}\right)\right) .
$$

Joining the estimates obtained so far for $I-I I I$ with (4.30) and enlarging the radius from $r$ to $2 r$ we arrive at

$$
f_{Q_{r}\left(z_{1}\right)}\left|\tau_{h} D u-\left(\tau_{h} D u\right)_{z_{1}, r}\right|^{2} d z \leqslant c f_{Q_{2 r}\left(z_{1}\right)}\left|w_{h}\right|^{2} d z
$$


where $w_{h} \in L^{\sigma_{1}}\left(Q_{\varrho}^{+}\left(z_{0}\right)\right)$ is defined in (4.31) and $\sigma_{1}=\sigma_{1}(n, N, L / v) \in\left[2,2+\frac{4}{n}\right]$ is the higher integrability exponent of $D^{2} u$ from Proposition 4.18 and $c=c(n, N, L)$. Note that (4.32) continues to hold in the case when $h \leqslant(r / 2)^{2}$; therefore (4.32) holds in any case, and we are in a situation similarly to the proof of Lemma 4.14, where we have the estimate (4.22). This allows us to repeat the proof from there line by line (i.e. we derive a bound for the sharp maximal function of $\tau_{h} D u$ in terms of the maximal function of $w_{h}$ and then apply the Hardy-Littlewood maximal theorem and the Fefferman-Stein theorem) to arrive at the following analogue of (4.24):

$$
f_{Q_{\varrho / 2}^{0}\left(z_{0}\right)}\left|\tau_{h} D u\right|^{\sigma} d z \leqslant c \int_{Q_{\varrho}^{0}\left(z_{0}\right)}\left|w_{h}\right|^{\sigma} d z+c\left(f_{Q_{\varrho / 2}^{0}\left(z_{0}\right)}\left|\tau_{h} D u\right| d z\right)^{\sigma},
$$

where $c=c(n, N, L)$ and $\sigma \in\left[2, \sigma_{1}\right]$. Recalling the definition of $w_{h}$ and applying Hölder's inequality and Lemma 4.19 to estimate the second integral appearing on the right-hand side we find

$$
\int_{Q_{\varrho / 2}^{0}}\left|\tau_{h} D u\right|^{\sigma} d z \leqslant c(n, N, L / v) h^{\frac{\sigma}{2}} \int_{Q_{\varrho}^{0}} \gamma^{\sigma}\left(1+|D u|^{\sigma}\right)+\left|D^{2} u\right|^{\sigma} d z .
$$

Now we apply Proposition 4.18 (note that $\sigma \leqslant \sigma_{1}$ ) to estimate the integral involving $D^{2} u$. This leads us to the assertion with $Q_{2 \varrho}^{0}$ instead of $Q_{\varrho}^{0}$. By a different choice of radii we can achieve the desired estimate on $Q_{\varrho}^{0}$.

\section{Singular set-dimension estimates}

In this section we shall prove Theorems 1.3 and 1.5. The basic strategy is the same in both cases. We shall construct solutions of certain regularized parabolic systems satisfying the assumptions of Section 4.1, respectively Section 4.2. This will be done by mollifying the original vector field $a$. Note that the mollifying procedure will be different in both configurations, since we need to assume some regularity in time (see (4.2) 4 ) in the lateral boundary situation, while in the initial boundary situation such a regularity assumption is unnecessary. The results achieved in Section 4 guarantee that the solutions of the regularized systems achieve higher differentiability and integrability properties. These estimates carry over, to a certain extend, to the original solution by a comparison and energy estimate. This procedure will lead us to a fractional differentiability result for $D u$ in space and time which immediately implies - with a refined version of Giusti's lemma - an estimate for the Hausdorff-dimension of the singular set; see Theorem 1.2. We start by considering

\subsection{The lateral boundary: Proof of Theorem 1.3}

In the following we shall concentrate our attention to a weak solution $u \in L^{2}\left(\Lambda_{1} ; W^{1,2}\left(B_{1}^{+}, \mathbb{R}^{N}\right)\right)$ of the following non-linear parabolic system

$$
u_{t}-\operatorname{div} a(z, D u)=g_{t} \quad \text { on } Q_{1}^{+},
$$

where $u=0$ on $\Gamma_{1}$. Here the vector field $a: Q_{1}^{+} \times \mathbb{R}^{N n} \rightarrow \mathbb{R}^{N n}$ satisfies the assumptions (1.2) and (1.3) with $\Omega_{T}$ replaced by $Q_{1}^{+}$. Note that the boundary values are only prescribed on $\Gamma_{1}$. Concerning the right-hand side $g$ we assume that

$$
D g \in C^{\beta, 0}\left(\overline{Q_{1}^{+}} ; \mathbb{R}^{N n}\right) \quad \text { and } \quad \partial_{t} g \in L^{2,2-2 \beta}\left(Q_{1}^{+} ; \mathbb{R}^{N n}\right) .
$$

\subsubsection{Smoothing}

The purpose of this section is to build up a family of regularized vector fields

$$
a_{h}: \overline{Q_{1 / 2}^{+}} \times \mathbb{R}^{N n} \rightarrow \mathbb{R}^{N n}, \quad|h| \in(0,1 / 2],
$$

starting from the one in (5.1). The construction of the vector fields $a_{h}$ is as follows. We first note that without loss of generality we can assume that the vector field $a$ is defined on

$$
a: \overline{B_{1}^{+}} \times \Lambda_{1} \times \mathbb{R}^{N n} \rightarrow \mathbb{R}^{N n}
$$


fulfilling the same assumptions (1.2) and (1.3) as $a$. Next, we extend $a$ to a vector field defined on $Q_{1} \times \mathbb{R}^{N n}-$ still denoted by $a$ - by an even reflection at the hyperplane $x_{n}=0$ as follows:

$$
a\left(x^{\prime}, x_{n}, t, w\right) \equiv \begin{cases}a\left(x^{\prime}, x_{n}, t, w\right) & \text { if } x_{n} \geqslant 0 \\ a\left(x^{\prime},-x_{n}, t, w\right) & \text { if } x_{n}<0 .\end{cases}
$$

It is easy to check that the extended vector field fulfills the assumptions (1.2) and (1.3) on $Q_{1}$ (with a possibly larger constant $2 L$ instead of $L$ ).

Now, having done the extension of the vector field $a$, we can explain the smoothing procedure. We fix a smooth, radially symmetric convolution kernel $\phi \in C_{0}^{\infty}\left(B_{1}\right)$ satisfying $\int_{B_{1}} \phi d x=1$ and a smooth, even convolution kernel $\psi \in C_{0}^{\infty}\left(\Lambda_{1}\right)$ with $\int_{\Lambda_{1}} \psi d t=1$. For $0<|h| \leqslant 1 / 2$ we then define the smooth vector field $a_{h}$ by

$$
\begin{aligned}
a_{h}(x, t, w) & \equiv \int_{\Lambda_{1}} \int_{B_{1}} a\left(x+|h| y, t+|h|^{2} \tau, w\right) \phi(y) \psi(\tau) d y d \tau \\
& =\frac{1}{|h|^{n+2}} \int_{\Lambda_{|h|^{2}}(t)} \int_{B_{|h|}(x)} a(y, \tau, w) \phi\left(\frac{y-x}{|h|}\right) \psi\left(\frac{\tau-t}{|h|^{2}}\right) d y d \tau,
\end{aligned}
$$

where $z=(x, t) \in Q_{1-|h|}$ and $w \in \mathbb{R}^{N n}$. Similarly to (3.16) in [15] we infer the following properties of the vector field $a_{h}$ :

$$
\left\{\begin{array}{l}
\left|a_{h}(z, w)\right|+(1+|w|)\left|\partial_{w} a_{h}(z, w)\right| \leqslant L(1+|w|), \\
\left\langle\partial_{w} a_{h}(z, w) \tilde{w},\left.\tilde{w}|\geqslant v| \tilde{w}\right|^{2},\right. \\
v|w-\tilde{w}|^{2} \leqslant\left\langle a_{h}(z, \tilde{w})-a_{h}(z, w), \tilde{w}-w\right\rangle, \\
\frac{v}{2}|w|^{2}-L^{2} / v \leqslant\left\langle a_{h}(z, w), w\right\rangle, \\
\left|a_{h}(z, w)-a(z, w)\right| \leqslant L \tilde{\theta}(|h|)(1+|w|), \\
\left|a_{h}(x, t, w)-a_{h}\left(x_{0}, t, w\right)\right| \leqslant c L \frac{\tilde{\theta}(|h|)}{|h|}\left|x-x_{0}\right|(1+|w|), \\
\left|a_{h}(x, t, w)-a_{h}\left(x, t_{0}, w\right)\right| \leqslant c L\left(\frac{\tilde{\theta}\left(|h|^{2}\right)}{|h|^{2}}\right)^{\frac{1+\vartheta}{2}}\left|t-t_{0}\right|^{\frac{1+\vartheta}{2}}(1+|w|),
\end{array}\right.
$$

for any $z, \tilde{z},\left(x_{0}, t\right),\left(x, t_{0}\right) \in Q_{1-|h|}, w, \tilde{w} \in \mathbb{R}^{N n}$ and $\vartheta \in(0,1)$, where the $c=c(n)$ is independent of $h$. The proof follows along the lines of [15]. The only difference occurs in the proof of (5.4) 7 where we argue as follows: We first compute

$$
\begin{aligned}
\left|\partial_{t} a_{h}(x, t, w)\right| & =\left|-\frac{1}{|h|^{n+4}} \int_{\Lambda_{|h|^{2}(t)}} \int_{B_{|h|}(x)} a(y, \tau, w) \phi\left(\frac{y-x}{|h|}\right) \psi^{\prime}\left(\frac{\tau-t}{|h|^{2}}\right) d y d \tau\right| \\
& =\left|\frac{1}{|h|^{2}} \int_{\Lambda_{1}} \int_{B_{1}} a\left(x+|h| y, t+|h|^{2} \tau, w\right) \phi(y) \psi^{\prime}(\tau) d y d \tau\right| \\
& =\left|\frac{1}{|h|^{2}} \int_{\Lambda_{1}} \int_{B_{1}}\left(a\left(x+|h| y, t+|h|^{2} \tau, w\right)-a(x+|h| y, t, w)\right) \phi(y) \psi^{\prime}(\tau) d y d \tau\right| \\
& \leqslant \frac{L}{|h|^{2}} \sup _{\Lambda_{1}}\left|\psi^{\prime}\right| \int_{-1}^{1} \int_{B_{1}} \tilde{\theta}\left(|h|^{2} \tau\right) \phi(y)(1+|w|) d y d \tau \leqslant c L \frac{\tilde{\theta}\left(|h|^{2}\right)}{|h|^{2}}(1+|w|),
\end{aligned}
$$

where we have used (1.2) 4 . This implies in particular that

$$
\left|a_{h}(x, t, w)-a_{h}\left(x, t_{0}, w\right)\right| \leqslant \sup _{s \in[0,1]}\left|\partial_{t} a_{h}\left(x, t+s\left(t_{0}-t\right), w\right)\right|\left|t-t_{0}\right| \leqslant c L \frac{\tilde{\theta}\left(|h|^{2}\right)}{|h|^{2}}\left|t-t_{0}\right|(1+|w|) .
$$


The case $|h|^{-2} \tilde{\theta}\left(|h|^{2}\right)\left|t-t_{0}\right| \leqslant 1$ leads us to (note that $\frac{1+\vartheta}{2}<1$ )

$$
\left|a_{h}(x, t, w)-a_{h}\left(x, t_{0}, w\right)\right| \leqslant c L\left(\frac{\tilde{\theta}\left(|h|^{2}\right)}{|h|^{2}}\left|t-t_{0}\right|\right)^{\frac{1+\vartheta}{2}}(1+|w|),
$$

while in the other case $|h|^{-2} \tilde{\theta}\left(|h|^{2}\right)\left|t-t_{0}\right| \geqslant 1$ we obtain

$$
\left|a_{h}(x, t, w)-a_{h}\left(x, t_{0}, w\right)\right| \leqslant 2 L(1+|w|) \leqslant 2 L\left(\frac{\tilde{\theta}\left(|h|^{2}\right)}{|h|^{2}}\left|t-t_{0}\right|\right)^{\frac{1+\vartheta}{2}}(1+|w|) .
$$

All together this proves $(5.4)_{7}$.

\subsubsection{A comparison estimate}

Here we shall derive an energy and a comparison estimate that will be crucial in the proof of the singular set estimate. By $u \in L^{2}\left(\Lambda_{1} ; W^{1,2}\left(B_{1}^{+} ; \mathbb{R}^{N}\right)\right)$ we denote a fixed solution of the non-linear parabolic system (5.1) satisfying $u=0$ on $\Gamma_{1}$. We shall keep on assuming that the vector field $a$ fulfills the assumptions (1.2) and (1.3).

For $0<R \leqslant 1 / 2$ we define $u_{h} \in L^{2}\left(\Lambda_{R^{2}} ; W^{1,2}\left(B_{R}^{+} ; \mathbb{R}^{N}\right)\right)$ to be the unique solution of the following CauchyDirichlet problem

$$
\begin{cases}\partial_{t} u_{h}-\operatorname{div} a_{h}\left(x, t, D u_{h}\right)=g_{t} & \text { in } Q_{R}^{+}, \\ u_{h}=u & \text { on } \partial_{\mathcal{P}} Q_{R}^{+},\end{cases}
$$

where $\left\{a_{h}\right\}, 0<|h| \leqslant 1 / 2$, denotes the family of vector fields constructed in (5.3). Then the following energy estimate holds

$$
\int_{Q_{R}^{+}}\left|D u_{h}\right|^{2} d z \leqslant c(L / v) \int_{Q_{R}^{+}}\left(1+|D u|^{2}\right) d z .
$$

Moreover, we have the following comparison estimate

$$
\sup _{t \in \Lambda_{R^{2}}} \int_{B_{R}^{+}}\left|\left(u_{h}-u\right)(\cdot, t)\right|^{2} d x+\int_{Q_{R}^{+}}\left|D u_{h}-D u\right|^{2} d z \leqslant c(L / v) \tilde{\theta}^{2}(|h|) \int_{Q_{R}^{+}}\left(1+|D u|^{2}\right) d z .
$$

In order to prove (5.6) and (5.7) we define $\chi_{\varepsilon} \in W^{1, \infty}(\mathbb{R})$ for $t \in \Lambda_{R^{2}}$ and $\varepsilon>0$ as follows: $\chi_{\varepsilon} \equiv 1$ on $(-\infty, t]$, $\chi_{\varepsilon} \equiv 0$ on $[t+\varepsilon, \infty)$ and $\chi_{\varepsilon}(\tau)=1-(\tau-t) / \varepsilon$ on $(t, t+\varepsilon)$. Now we proceed formally by testing the parabolic systems for $u$, respectively $u_{h}$ with $\varphi(x, t)=\chi_{\varepsilon}(t)\left(u_{h}(x, t)-u(x, t)\right)$ and then letting $\varepsilon \downarrow 0$. The argument can be justified by the use of Steklov averages. Note that the test-function $\varphi$ is admissible in (5.1) and (5.5) since $u_{h}$ and $u$ agree on the parabolic boundary $\partial_{\mathcal{P}} Q_{R}^{+}$. Now, testing (5.1) and (5.5) as described before and then taking the difference we obtain for a.e. $t \in \Lambda_{R^{2}}$

$$
\frac{1}{2} \int_{B_{R}^{+}}\left|\left(u_{h}-u\right)(\cdot, t)\right|^{2} d x+\int_{-R^{2}}^{t} \int_{B_{R}^{+}}\left\langle a_{h}\left(\cdot, D u_{h}\right)-a(\cdot, D u), D u_{h}-D u\right\rangle d x d \tau=0 .
$$

To prove the energy estimate we let $t \uparrow R^{2}$. Noting that the first term on the left-hand side is non-negative and also using (5.4) 4 , we find that

$$
\begin{aligned}
\int_{Q_{R}^{+}}\left(\frac{1}{2} v\left|D u_{h}\right|^{2}-L^{2} / v\right) d z \leqslant & \int_{Q_{R}^{+}}\left\langle a_{h}\left(\cdot, D u_{h}\right), D u_{h}\right\rangle d z \\
& \leqslant \int_{Q_{R}^{+}}\left\langle a_{h}\left(\cdot, D u_{h}\right), D u\right\rangle d z+\int_{Q_{R}^{+}}\left\langle a_{h}(\cdot, D u), D u_{h}-D u\right\rangle d z=: I+I I,
\end{aligned}
$$


with the obvious meaning of $I$ and $I I$. Using the growth (5.4) $)_{1}$ of $a_{h}$ and Young's inequality (with $\varepsilon>0$ to be chosen later) we find

$$
|I| \leqslant L \int_{Q_{R}^{+}}\left(1+\left|D u_{h}\right|\right)|D u| d z \leqslant \varepsilon \int_{Q_{R}^{+}}\left|D u_{h}\right|^{2} d z+\left(L+L^{2} \varepsilon^{-1}\right) \int_{Q_{R}^{+}}\left(1+|D u|^{2}\right) d z
$$

as well as

$$
|I I| \leqslant L \int_{Q_{R}^{+}}(1+|D u|)\left(\left|D u_{h}\right|+|D u|\right) d z \leqslant \varepsilon \int_{Q_{R}^{+}}\left|D u_{h}\right|^{2} d z+2\left(L+L^{2} \varepsilon^{-1}\right) \int_{Q_{R}^{+}}\left(1+|D u|^{2}\right) d z .
$$

Inserting the preceding estimates for $I$ and $I I$ above and choosing $\varepsilon>0$ small enough, we obtain the desired energy estimate (5.6) with a constant $c=c(L / v)$. To prove the comparison estimate we first use $(5.4)_{3}$ to obtain

$$
I(t)=\int_{-R^{2}}^{t} \int_{B_{R}^{+}}\left|D u-D u_{h}\right|^{2} d x d \tau \leqslant \frac{1}{v} \int_{-R^{2}}^{t} \int_{B_{R}^{+}}\left\langle a_{h}\left(\cdot, D u_{h}\right)-a_{h}(\cdot, D u), D u_{h}-D u\right\rangle d x d \tau,
$$

for a.e. $t \in \Lambda_{R^{2}}$. Combining this with (5.8), using (5.4) 5 and Young's inequality, we find

$$
\begin{aligned}
\frac{1}{2} \int_{B_{R}^{+}}\left|\left(u_{h}-u\right)(\cdot, t)\right|^{2} d x+v I(t) & \leqslant \int_{-R^{2}}^{t} \int_{B_{R}^{+}}\left\langle a(\cdot, D u)-a_{h}(\cdot, D u), D u_{h}-D u\right\rangle d x d \tau \\
& \leqslant L \tilde{\theta}(|h|) \int_{-R^{2}}^{t} \int_{B_{R}^{+}}(1+|D u|)\left|D u_{h}-D u\right| d x d \tau \\
& \leqslant \frac{v}{2} I(t)+L^{2} v^{-1} \tilde{\theta}^{2}(|h|) \int_{Q_{R}^{+}}\left(1+|D u|^{2}\right) d z .
\end{aligned}
$$

We absorb $\frac{v}{2} I(t)$ on the left-hand side, then take the supremum over $t \in \Lambda_{R^{2}}$ in the first term and finally let $t \uparrow R^{2}$ in the second one. This procedure gives the desired comparison estimate (5.7).

\subsubsection{Fractional estimates}

In this section we will establish higher fractional differentiability of $D u$ with respect to the spatial directions and the time direction up to the lateral boundary. This will be achieved by a comparison argument, which utilizes the up to the boundary higher differentiability of the associated regularized problem.

Lemma 5.1. Let $u \in L^{2}\left(\Lambda_{1} ; W^{1,2}\left(B_{1}^{+} ; \mathbb{R}^{N}\right)\right)$ be a weak solution of the non-linear parabolic system (5.1) in $Q_{1}^{+}$with $u=0$ on $\Gamma_{1}$ where the vector field a satisfies the hypotheses (1.2) and $g_{t} \in \mathcal{N}^{0, \xi ; 2}\left(Q_{1}^{+} ; \mathbb{R}^{N}\right)$ for some $\xi \in(0,1)$. Then for any $0<r \leqslant 1 / 16$ there holds

$$
\iint_{\Lambda_{r^{2}}} \int_{B_{r}^{+}} \frac{|D u(x, t)-D u(y, t)|^{2}}{|x-y|^{n+2 s}} d x d y d t<\infty, \quad \forall s \in(0, \beta) .
$$

Proof. First, we will derive an estimate for the integral of the finite differences $\tau_{h}^{\alpha}[D u], \alpha=1, \ldots, n$, in terms of the increment $|h|$. For this we fix $h \in \mathbb{R}$ such that $0<|h| \leqslant r$ where we take $h>0$ when dealing with the case $\alpha=n$. By

$$
u_{h} \in L^{2}\left(\Lambda_{(1 / 2)^{2}} ; W^{1,2}\left(B_{1 / 2}^{+} ; \mathbb{R}^{N}\right)\right)
$$

we denote the unique solution of the Cauchy-Dirichlet problem (5.5) in $Q_{1 / 2}^{+}$. Moreover, for $s \in(0, \beta)$ we fix $0<\vartheta<$ $\min \left\{\frac{\beta-s}{1-\beta}, \xi\right\}$. We note that due to $(5.4)_{1},(5.4)_{2},(5.4)_{6},(5.4)_{7}$ and the assumption $\tilde{\theta}(|h|) \leqslant|h|^{\beta}$, the vector fields $a_{h}$ 
are of the type of the vector fields considered in (4.1). In particular, the assumptions (4.2) 3 and (4.2) 4 are satisfied with

$$
\gamma=\gamma(h)=c(n)\left[\frac{\tilde{\theta}(|h|)}{|h|}+\left(\frac{\tilde{\theta}\left(|h|^{2}\right)}{|h|^{2}}\right)^{\frac{1+\vartheta}{2}}\right] \leqslant c(n)|h|^{(\beta-1)(1+\vartheta)} .
$$

Therefore, the a priori estimate from Theorem 4.2 applies to $u_{h}$ such that we have $D^{2} u_{h} \in L^{2}\left(Q_{4 r}^{+} ; \mathbb{R}^{N n^{2}}\right)$ with the estimate

$$
\begin{aligned}
\int_{Q_{4 r}^{+}}\left|D^{2} u_{h}\right|^{2} d z & \leqslant c\left(r^{-2}+\gamma^{2}\right) \int_{Q_{8 r}^{+}}\left(1+\left|D u_{h}\right|^{2}\right) d z+c\left\|g_{t}\right\|_{\mathcal{N}^{0, \xi ; 2}\left(Q_{8 r}^{+}\right)}^{2} \\
& \leqslant c\left(r^{-2}+|h|^{2(\beta-1)(1+\vartheta)}\right) \int_{Q_{8 r}^{+}}\left(1+|D u|^{2}\right) d z+c\left\|g_{t}\right\|_{\mathcal{N}^{0, \xi ; 2}\left(Q_{8 r}^{+}\right)}^{2},
\end{aligned}
$$

where in the last line we have used the energy estimate (5.6) and (5.9). Note that $c=c(n, N, L / v, \vartheta)$ and $c \rightarrow \infty$ when $\vartheta \downarrow 0$. With the help of (2.7), this estimate yields for the finite differences $\tau_{h}^{\alpha}[D u], \alpha=1, \ldots, n$, that

$$
\int_{Q_{2 r}^{+}}\left|\tau_{h}^{\alpha}\left[D u_{h}\right]\right|^{2} d z \leqslant c(n)|h|^{2} \int_{Q_{4 r}^{+}}\left|D^{2} u_{h}\right|^{2} d z \leqslant c|h|^{2 \beta-2 \vartheta(1-\beta)}\left[r^{-2} \int_{Q_{8 r}^{+}}\left(1+|D u|^{2}\right) d z+\left\|g_{t}\right\|_{\mathcal{N}^{0, \xi ; 2}\left(Q_{8 r}^{+}\right)}^{2}\right] .
$$

In order to derive a similar estimate for $\tau_{h}^{\alpha}[D u]$ we use the following comparison argument:

$$
\begin{aligned}
\int_{Q_{2 r}^{+}}\left|\tau_{h}^{\alpha}[D u]\right|^{2} d z \leqslant & 3 \int_{Q_{2 r}^{+}}\left|\tau_{h}^{\alpha}\left[D u_{h}\right]\right|^{2} d z+3 \int_{Q_{2 r}^{+}}\left|D u_{h}\left(x+h e_{\alpha}, t\right)-D u\left(x+h e_{\alpha}, t\right)\right|^{2} d z \\
& +3 \int_{Q_{2 r}^{+}}\left|D u_{h}(x, t)-D u(x, t)\right|^{2} d z .
\end{aligned}
$$

Taking into account that $|h| \leqslant r$ and using the comparison estimate (5.7) we can control the last two integrals by

$$
\int_{Q_{4 r}^{+}}\left|D u_{h}-D u\right|^{2} d z \leqslant c(n, L / v)|h|^{2 \beta} \int_{Q_{8 r}^{+}}\left(1+|D u|^{2}\right) d z
$$

Combining this with the preceding estimate for $\tau_{h}^{\alpha}\left[D u_{h}\right]$ we finally arrive at

$$
\int_{Q_{2 r}^{+}}\left|\tau_{h}^{\alpha}[D u]\right|^{2} d z \leqslant c|h|^{2 \beta-2 \vartheta(1-\beta)}\left[r^{-2} \int_{Q_{8 r}^{+}}\left(1+|D u|^{2}\right) d z+\left\|g_{t}\right\|_{\mathcal{N}^{0, \xi ; 2}\left(Q_{8 r}^{+}\right)}^{2}\right],
$$

where $c=c(n, N, L / v, \vartheta)$. It is worth mentioning that $c \rightarrow \infty$ when $\vartheta \downarrow 0$, i.e. when $\xi \downarrow 0$ or $s \uparrow \beta$. Note that we have taken $h>0$ in the case $\alpha=n$. Since $\beta-\vartheta(1-\beta)>s$ by our choice of $\vartheta$ the application of Lemma 2.5(ii) now yields $D u \in W^{s, 0,2}\left(Q_{r}^{+} ; \mathbb{R}^{N n}\right)$. Recalling that $s \in(0, \beta)$ was arbitrary this proves the assertion of the lemma.

Similarly we can show fractional differentiability of $D u$ with respect to time.

Lemma 5.2. Let $u \in L^{2}\left(\Lambda_{1} ; W^{1,2}\left(B_{1}^{+} ; \mathbb{R}^{N}\right)\right)$ be a weak solution of the non-linear parabolic system (5.1) in $Q_{1}^{+}$with $u=0$ on $\Gamma_{1}$ where the vector field a satisfies the hypotheses $(1.2)$ and $g_{t} \in \mathcal{N}^{0, \xi ; 2}\left(Q_{1}^{+} ; \mathbb{R}^{N}\right)$ for some $\xi \in(0,1)$. Then for any $0<r \leqslant 1 / 8$ we have

$$
\iint_{\Lambda_{r^{2}}} \int_{\Lambda_{r^{2}}} \int_{B_{r}^{+}} \frac{|D u(x, t)-D u(x, \tau)|^{2}}{|t-\tau|^{1+2 s}} d t d \tau d x<\infty, \quad \forall s \in\left(0, \frac{\beta}{2}\right) .
$$


Proof. We fix $\lambda \in \mathbb{R}$ such that $0<|\lambda| \leqslant r^{2}$ and define $h=|\lambda|^{\frac{1}{2}}$. Again we denote by

$$
u_{h} \in L^{2}\left(\Lambda_{(1 / 2)^{2}} ; W^{1,2}\left(B_{1 / 2}^{+} ; \mathbb{R}^{N}\right)\right)
$$

the unique solution of the Cauchy-Dirichlet problem (5.5) on $Q_{1 / 2}^{+}$and for $s \in(0, \beta)$ we fix $0<\vartheta<\min \left\{\frac{\beta-s}{1-\beta}, \xi\right\}$. From the proof of Lemma 5.1 we recall that the vector fields $\left\{a_{h}\right\}$ are of the type of the vector fields considered in (4.1) with $\gamma=\gamma(h) \leqslant c(n) h^{(\beta-1)(1+\vartheta)}=c(n)|\lambda|^{\frac{\beta-1}{2}(1+\vartheta)}$ (see (5.9)). Therefore, we can apply Corollary 4.9 to $u_{h}$ to infer

$$
\begin{aligned}
\int_{Q_{2 r}^{+}}\left|\tau_{\lambda}\left[D u_{h}\right]\right|^{2} d z & \leqslant c|\lambda|\left[\left(r^{-2}+\gamma^{2}\right) \int_{Q_{4 r}^{+}}\left(1+\left|D u_{h}\right|^{2}\right) d z+\left\|g_{t}\right\|_{\mathcal{N}^{0, \xi ; 2}\left(Q_{4 r}^{+}\right)}^{2}\right] \\
& \leqslant c|\lambda|^{\beta-\vartheta(1-\beta)}\left[r^{-2} \int_{Q_{4 r}^{+}}\left(1+|D u|^{2}\right) d z+\left\|g_{t}\right\|_{\mathcal{N}^{0, \xi ; 2}\left(Q_{4 r}^{+}\right)}^{2}\right],
\end{aligned}
$$

where we have used again in the last line the energy estimate (5.6) and $c=c(n, N, L / v, \vartheta)$ with $c \rightarrow \infty$ when $\vartheta \downarrow 0$. In order to derive a similar estimate for $\tau_{\lambda}[D u]$ we once again use a comparison argument:

$$
\begin{aligned}
\int_{Q_{r}^{+}}\left|\tau_{\lambda}[D u]\right|^{2} d z \leqslant & 3 \int_{Q_{r}^{+}}\left|\tau_{\lambda}\left[D u_{h}\right]\right|^{2} d z+3 \int_{Q_{r}^{+}}\left|D u_{h}(x, t+\lambda)-D u(x, t+\lambda)\right|^{2} d z \\
& +3 \int_{Q_{r}^{+}}\left|D u_{h}(x, t)-D u(x, t)\right|^{2} d z .
\end{aligned}
$$

Taking into account that $|\lambda| \leqslant r^{2}$ and using again the comparison estimate (5.7) we can control the last two integrals by

$$
\int_{Q_{2 r}^{+}}\left|D u_{h}-D u\right|^{2} d z \leqslant c(n, N, L / v)|\lambda|^{\beta} \int_{Q_{4 r}^{+}}\left(1+|D u|^{2}\right) d z .
$$

Combining this with the estimate for $\tau_{\lambda}\left[D u_{h}\right]$ we finally arrive at

$$
\int_{Q_{r}^{+}}\left|\tau_{\lambda}[D u]\right|^{2} d z \leqslant c|\lambda|^{\beta-\vartheta(1-\beta)}\left[r^{-2} \int_{Q_{4 r}^{+}}\left(1+|D u|^{2}\right) d z+\left\|g_{t}\right\|_{\mathcal{N}^{0, \xi ; 2}\left(Q_{4 r}^{+}\right)}^{2}\right],
$$

where $c=c(n, N, L / v, \vartheta)$. As before, we mention that $c \rightarrow \infty$ when $\vartheta \downarrow 0$, i.e. when $\xi \downarrow 0$ or $s \uparrow \beta$. By our choice of $\vartheta$ the application of Lemma 2.5(i) is possible and yields $D u \in W^{0, s, 2}\left(Q_{r}^{+} ; \mathbb{R}^{N n}\right)$, proving the assertion of the lemma.

At the end of this section we briefly summarize on the achievements so far. Combining Lemmas 5.1 and 5.2 with their interior analogue (see [16, Lemmas 9.4 and 9.5]) via the flattening of the boundary procedure described in Section 2.1, we arrive at the following global differentiability result:

Theorem 5.3. Let $u \in L^{2}\left(0, T ; W^{1,2}\left(\Omega ; \mathbb{R}^{N}\right)\right)$ be a weak solution of the non-linear parabolic system (1.1) under the assumptions (1.2) where $u=g$ on the lateral part $\partial_{\mathrm{lat}} \Omega_{T}$ of the parabolic boundary and $g_{t} \in \mathcal{N}^{0, \xi ; 2}\left(Q_{1}^{+} ; \mathbb{R}^{N}\right)$ for some $\xi \in(0,1)$. Then for any $d \in(0, T / 2)$ and any $s \in(0, \beta)$ there holds

$$
D u \in W^{s, \frac{s}{2}, 2}\left(\Omega \times(d, T-d) ; \mathbb{R}^{N n}\right), \quad \forall s<\beta .
$$

\subsubsection{Proof of Theorem 1.3}

Now, we are in the position to prove the existence of regular points on the lateral boundary. The first assertion of Theorem 1.3 is an immediate consequence of Theorem 5.3, i.e. the fact that $D u \in W^{s, s / 2,2}$ for any $s \in(0, \beta)$. 
The gain of the $\delta$-improvement in (1.7) will be slightly more involved. Here we will use a higher integrability argument to replace in several estimates the $L^{2}$-norm by an $L^{\sigma}$-norm for some $\sigma>2$, such that we will come up with $D u \in W^{s, s / 2, \sigma}$.

Proof of Theorem 1.3. Here, we will show that

$$
\operatorname{dim}_{\mathcal{P}}\left(\partial_{\text {lat }} \Omega_{T} \backslash \operatorname{Reg}_{\mathcal{P}} u\right)<n+1
$$

which implies that $\mathcal{H}_{\mathcal{P}}^{n+1}$-almost every lateral boundary point is a regular point of $D u$. By the flattening of the boundary procedure introduced in Section 2.1 we can cover $\partial_{\text {lat }} \Omega_{T}$ by a finite number of cylinders $Q_{\varrho}\left(z_{i}\right), i=1, \ldots, \mathcal{M}$, such that it suffices to consider the model problem (2.2) on $Q_{1}^{+}$and to establish the estimate for the dimension of the singular set $\Gamma_{1 / 2} \backslash \operatorname{Reg}_{\mathcal{P}} u$; to be more precise that $\operatorname{dim}_{\mathcal{P}}\left(\Gamma_{1 / 2} \backslash \operatorname{Reg}_{\mathcal{P}} u\right)<n+1$. From the characterization of regular points in Theorem 1.2 (transformed to the model situation (2.2)) we already know that $\Gamma_{1 / 2} \backslash \operatorname{Reg}_{\mathcal{P}} u \subset$ $\Gamma_{1 / 2} \cap\left(\Sigma^{1} \cup \Sigma^{2}\right)$. We now apply Theorem 5.3 to infer that $D u \in W^{s, s / 2,2}\left(Q_{1 / 2}^{+} ; \mathbb{R}^{N n}\right)$ for any $s \in(0, \beta)$. Therefore, Lemma 2.6 yields

$$
\operatorname{dim}_{\mathcal{P}}\left(\Gamma_{1 / 2} \cap\left(\Sigma^{1} \cup \Sigma^{2}\right)\right) \leqslant n+2-2 \beta<n+1,
$$

whenever $\beta>\frac{1}{2}$. This proves the first assertion of Theorem 1.3.

In the following we shall improve this bound. The strategy here is to improve the comparison estimate (5.7) and the fractional differentiability properties from Theorem 5.3, in the sense that in the $L^{2}$-norm is replaced by the $L^{\sigma}$-norm for some small values of $\sigma>2$. With this respect we can assume that $g_{t} \in \mathcal{N}^{0, \xi ; \sigma_{1}}\left(Q_{1}^{+} ; \mathbb{R}^{N}\right)$ for some $\xi \in(0,1)$ and some $\sigma_{1}>2$. Our aim now is to determine $\delta>0$ such that $\beta>1 / 2-\delta$ implies that

$$
\operatorname{dim}_{\mathcal{P}}\left(\Gamma_{r} \cap\left(\Sigma^{1} \cup \Sigma^{2}\right)\right)<n+1,
$$

for some $r>0$ to be determined later but such that the pull back of the $Q_{r}$ 's via the flattening of procedure covers $\partial_{\text {lat }} \Omega_{T}$. From the up to the lateral boundary higher integrability in Lemma 3.1 we infer that there exists $\sigma_{2}=\sigma_{2}(n, L / \nu) \in\left(2, \sigma_{1}\right]$ such that

$$
D u \in L^{\sigma_{2}}\left(Q_{1 / 2}^{+} ; \mathbb{R}^{N n}\right) .
$$

Now, we let $\left\{a_{h}\right\}$ be the vector fields defined in Section 5.1 .1 and $u_{h} \in L^{2}\left(\Lambda_{(1 / 2)^{2}} ; W^{1,2}\left(B_{1 / 2}^{+} ; \mathbb{R}^{N}\right)\right)$ the unique solution of the initial Cauchy-Dirichlet problem (5.5) in $Q_{1 / 2}^{+}$. Taking the difference of systems (5.1) and (5.5) we infer

$$
\partial_{t}\left(u_{h}-u\right)-\operatorname{div}\left(a_{h}\left(z, D u_{h}\right)-a_{h}(z, D u)\right)=\operatorname{div}\left(a_{h}(z, D u)-a(z, D u)\right)
$$

on $Q_{1 / 2}^{+}$. We now set

$$
\tilde{\mathcal{A}}(h)(z):=\int_{0}^{1} \partial_{w} a_{h}\left(z, D u+s\left(D u_{h}-D u\right)\right) d s,
$$

and observe that $a_{h}\left(z, D u_{h}\right)-a_{h}(z, D u)=\tilde{\mathcal{A}}(h)\left(D u_{h}-D u\right)$. Therefore, we find that $u_{h}-u$ is a weak solution of the following linear initial Cauchy-Dirichlet problem:

$$
\begin{cases}\partial_{t}\left(u_{h}-u\right)-\operatorname{div}\left(\tilde{\mathcal{A}}(h) D\left(u_{h}-u\right)\right)=\operatorname{div}\left(a_{h}(\cdot, D u)-a(\cdot, D u)\right) & \text { in } Q_{1 / 2}^{+}, \\ u_{h}-u=0 & \text { on } \partial_{\mathcal{P}} Q_{1 / 2}^{+} .\end{cases}
$$

From our assumptions (5.4) $)_{1}$ and (5.4) $)_{2}$ we know that $\langle\tilde{\mathcal{A}}(h)(z) w, w\rangle \geqslant v|w|^{2}$ and also $|\tilde{\mathcal{A}}(h)(z)| \leqslant L$ for any choice of $w \in \mathbb{R}^{N n}$ and $z \in Q_{1 / 2}^{+}$. Therefore, we can apply Lemma 3.1 with the right-hand side $b(z)=a_{h}(z, D u)-a(z, D u) \in$ $L^{\sigma_{2}}\left(Q_{1 / 2}^{+} ; \mathbb{R}^{N n}\right)$ (see $(5.4)_{1},(1.2)_{1}$ and (5.10)) to infer the existence of $\sigma_{3}=\sigma_{3}(n, L / v) \in\left(2, \sigma_{2}\right]$ such that $D u_{h}-$ $D u \in L^{\sigma_{3}}\left(Q_{1 / 4}^{+} ; \mathbb{R}^{N n}\right)$. Moreover, for any choice of $\sigma \in\left(2, \sigma_{3}\right]$ holds 


$$
\int_{Q_{1 / 4}^{+}}\left|D u_{h}-D u\right|^{\sigma} d z \leqslant c\left(\int_{Q_{1 / 2}^{+}}\left|D u_{h}-D u\right|^{2} d z\right)^{\frac{\sigma}{2}}+c \int_{Q_{1 / 2}^{+}}\left|a_{h}(z, D u)-a(z, D u)\right|^{\sigma} d z,
$$

where $c=c(n, N, L / v)$. In the following we shall estimate the integrals appearing on the right-hand side of the preceding inequality. For the first we use the comparison estimate (5.7) and Hölder's inequality to infer

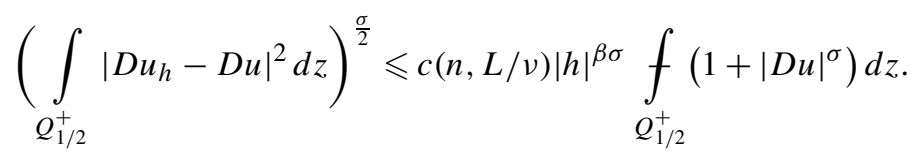

The second integral can be treated using the pointwise estimate $(5.4)_{5}$ to obtain

$$
\int_{Q_{1 / 2}^{+}}\left|a_{h}(\cdot, D u)-a(\cdot, D u)\right|^{\sigma} d z \leqslant c(n, L / v)|h|^{\beta \sigma} \int_{Q_{1 / 2}^{+}}\left(1+|D u|^{\sigma}\right) d z .
$$

Inserting this above we arrive at

$$
\int_{Q_{1 / 4}^{+}}\left|D u_{h}-D u\right|^{\sigma} d z \leqslant c(n, L / v)|h|^{\beta \sigma} \int_{Q_{1 / 2}^{+}}\left(1+|D u|^{\sigma}\right) d z,
$$

which is the desired improvement of the comparison estimate (5.7) we were looking for. At this stage we also note that the last inequality implies the energy bound:

$$
\int_{Q_{1 / 4}^{+}}\left|D u_{h}\right|^{\sigma} d z \leqslant c(n, L / v) \int_{Q_{1 / 2}^{+}}\left(1+|D u|^{\sigma}\right) d z .
$$

Next, we are going to improve the $W^{s, \frac{s}{2}, 2}$-estimate from Theorem 5.3, exploiting the higher integrability of $D^{2} u_{h}$. We first fix $s \in(0, \beta)$ and $0<\vartheta<\min \left\{\frac{\beta-s}{1-\beta}, \xi\right\}$. Applying Proposition 4.15, we infer the existence of $\sigma_{4}=\sigma_{4}(n, L / \nu) \in$ $\left(2, \sigma_{3}\right]$, such that $\left|D^{2} u_{h}\right| \in L^{\sigma_{4}}\left(Q_{1 / 4}^{+}\right)$. Moreover, for any choice of $\sigma \in\left(2, \sigma_{4}\right]$ we have

$$
\begin{aligned}
\int_{Q_{1 / 8}^{+}}\left|D^{2} u_{h}\right|^{\sigma} d z & \leqslant c\left(1+\gamma^{\sigma}\right) \int_{Q_{1 / 4}^{+}}\left(1+\left|D u_{h}\right|^{\sigma}\right) d z+c\left\|g_{t}\right\|_{\mathcal{N}^{0, \xi ; \sigma}\left(Q_{1 / 4}^{+}\right)}^{\sigma} \\
& \leqslant c|h|^{\sigma(\beta-1)(1+\vartheta)} \int_{Q_{1 / 2}^{+}}\left(1+|D u|^{\sigma}\right) d z+c\left\|g_{t}\right\|_{\mathcal{N}^{0, \xi ; \sigma}\left(Q_{1 / 2}^{+}\right)}^{\sigma},
\end{aligned}
$$

where we have used in the last line the energy bound (5.13) and $\gamma \leqslant c(n)|h|^{(\beta-1)(1+\vartheta)}$ from (5.9). Note that $c=$ $c(n, N, L / \nu, \vartheta)$ with $c \rightarrow \infty$ when $\vartheta \downarrow 0$. This yields for $\alpha=1, \ldots, n$ and $|h| \leqslant 1 / 16$ with $h>0$ when $\alpha=n$ that

$$
\int_{Q_{1 / 16}^{+}}\left|\tau_{h}^{\alpha}\left[D u_{h}\right]\right|^{\sigma} d z \leqslant c|h|^{\sigma[\beta-\vartheta(1-\beta)]}\left[\int_{Q_{1 / 2}^{+}}\left(1+|D u|^{\sigma}\right) d z+\left\|g_{t}\right\|_{\mathcal{N}^{0, \xi ; \sigma}\left(Q_{1 / 2}^{+}\right)}^{\sigma}\right] .
$$

This estimate can now be carried over to $D u$ by the comparison argument we used in the proof of Lemma 5.1. Indeed, combining (5.12) and the preceding estimate we obtain

$$
\begin{aligned}
\int_{Q_{1 / 16}^{+}}\left|\tau_{h}^{\alpha}[D u]\right|^{\sigma} d z \leqslant & 3^{\sigma-1}\left[\int_{Q_{1 / 16}^{+}}\left|\tau_{h}^{\alpha}\left[D u_{h}\right]\right|^{\sigma} d z+\int_{Q_{1 / 16}^{+}}\left|D u_{h}-D u\right|^{\sigma} d z\right. \\
& \left.+\int_{Q_{1 / 16}^{+}}\left|D u_{h}\left(x+h e_{\alpha}, t\right)-D u\left(x+h e_{\alpha}, t\right)\right|^{\sigma} d z\right]
\end{aligned}
$$




$$
\leqslant c|h|^{\sigma[\beta-\vartheta(1-\beta)]}\left[\int_{Q_{1 / 2}^{+}}\left(1+|D u|^{\sigma}\right) d z+\left\|g_{t}\right\|_{\mathcal{N}^{0, \xi ; \sigma}\left(Q_{1 / 2}^{+}\right)}^{\sigma}\right] .
$$

Finally, we consider the time-direction. We will attain an estimate for the $L^{\sigma}$-norm of $\left|\tau_{\lambda}[D u]\right|$ for $0<|\lambda| \leqslant(1 / 8)^{2}$ with the help of the comparison map $u_{h}$ with the choice $h=|\lambda|^{\frac{1}{2}}$. From Corollary 4.16 and Hölder's inequality we infer the existence of $\sigma_{5}=\sigma_{5}(n, L / \nu) \in\left(2, \sigma_{4}\right]$ such that for any $\sigma \in\left(2, \sigma_{5}\right]$ there holds

$$
\begin{aligned}
\int_{Q_{1 / 8}^{+}}\left|\tau_{\lambda}\left[D u_{h}\right]\right|^{\sigma} d z & \leqslant c|\lambda|^{\frac{\sigma}{2}}\left[\left(1+\gamma^{\sigma}\right) \int_{Q_{1 / 4}^{+}}\left(1+\left|D u_{h}\right|^{\sigma}\right) d z+\left\|g_{t}\right\|_{\mathcal{N}^{0, \xi ; \sigma}\left(Q_{1 / 4}^{+}\right)}^{\sigma}\right] \\
& \leqslant c|\lambda|^{\frac{\sigma}{2}(\beta-\vartheta(1-\beta))}\left[\int_{Q_{1 / 2}^{+}}\left(1+|D u|^{\sigma}\right) d z+\left\|g_{t}\right\|_{\mathcal{N}^{0, \xi ; \sigma}\left(Q_{1 / 2}^{+}\right)}^{\sigma}\right],
\end{aligned}
$$

where in the last line we have used again the energy bound (5.13) and (5.9) in the form $\gamma \leqslant c(n) h^{(\beta-1)(1+\vartheta)}=$ $c(n)|\lambda|^{\frac{\beta-1}{2}(1+\vartheta)}$.

Joining the comparison estimate (5.12) with the preceding estimate we obtain, completely similar to the proof of (5.15), that

$$
\begin{aligned}
& \int_{Q_{1 / 16}^{+}}\left|\tau_{\lambda}[D u]\right|^{\sigma} d z \leqslant 3^{\sigma-1}\left[\int_{Q_{1 / 16}^{+}}\left|\tau_{\lambda}\left[D u_{h}\right]\right|^{\sigma} d z+\int_{Q_{1 / 16}^{+}}\left|D u_{h}-D u\right|^{\sigma} d z\right. \\
&\left.+\int_{Q_{1 / 16}^{+}}\left|D u_{h}(x, t+\lambda)-D u(x, t+\lambda)\right|^{\sigma} d z\right] \\
& \leqslant c|\lambda|^{\frac{\sigma}{2}(\beta-\vartheta(1-\beta))}\left[\int_{Q_{1 / 2}^{+}}\left(1+|D u|^{\sigma}\right) d z+\left\|g_{t}\right\|_{\mathcal{N}^{0, \xi ; \sigma}\left(Q_{1 / 2}^{+}\right)}^{\sigma}\right]
\end{aligned}
$$

where $c=c(n, N, L / v, \vartheta)$. Combining (5.15) and (5.16) and recalling that $\vartheta<\frac{\beta-s}{1-\beta}$, we infer from Lemma 2.5 that $D u \in W^{s, s / 2, \sigma}\left(Q_{1 / 32}^{+} ; \mathbb{R}^{N n}\right)$. Since $s \in(0, \beta)$ was arbitrary we conclude that $D u \in W^{s, s / 2, \sigma}\left(Q_{1 / 32}^{+} ; \mathbb{R}^{N n}\right)$ for any $s \in(0, \beta)$ and $\sigma \in\left(2, \sigma_{5}\right]$.

At this stage we perform the choice of the constants. We first choose $\sigma=\sigma_{5}=\sigma_{5}(n, L / v)$. Next, we choose $\delta$ in (1.7) such that

$$
\delta \equiv \frac{1}{4}-\frac{1}{2 \sigma}>0 .
$$

Note that the dependencies of $\delta$ from the structural parameters are the same as for $\sigma$. Since $\beta>1 / 2-\delta$ we now choose $s \in(1 / 2-\delta, \beta)$. Applying Lemma 2.6(i) we infer that $\operatorname{dim}_{\mathcal{P}}\left(\Gamma_{1 / 32} \cap\left(\Sigma^{1} \cup \Sigma^{2}\right)\right)<n+2-s \sigma<n+1$. The last inequality follows from the fact that $s \sigma>(1 / 2-\delta) \sigma=\sigma / 4+1 / 2>1$. This finishes the proof of Theorem 1.3.

With the proof of Theorem 1.3 we have completed our considerations concerning the regularity properties of weak solutions to non-linear parabolic systems and their corresponding singular sets at the lateral boundary. Therefore we finally turn our attention to the dimension estimate for the singular set at

\subsection{The initial boundary: Proof of Theorem 1.5}

In the following we shall consider a weak solution $u \in L^{2}\left(0, T ; W^{1,2}\left(\Omega, \mathbb{R}^{N}\right)\right)$ of the following non-linear parabolic system

$$
u_{t}-\operatorname{div} a(z, D u)=0 \quad \text { on } \Omega_{T},
$$

where $u(\cdot, 0)=0$ on $\Omega$ and the vector field $a: \Omega_{T} \times \mathbb{R}^{N n} \rightarrow \mathbb{R}^{N n}$ satisfies the assumptions (1.2) $1-(1.2)_{3}$ and (1.3). 


\subsubsection{Smoothing}

As in the lateral boundary situation we build up a family of regularized vector fields. In contrast to Section 5.1.1 the smoothing will now be performed only with respect to the space variable $x$. The precise construction is as follows: We fix a point $z_{0} \in \Omega_{0}$, a parabolic cylinder $Q_{2 \varrho}^{0}\left(z_{0}\right) \subset \Omega_{T}$ and a smooth, radially symmetric, non-negative convolution kernel $\phi \in C_{0}^{\infty}\left(B_{1}\right)$ satisfying $\int_{B_{1}} \phi d x=1$. For $0<|h| \leqslant \min \{\varrho, 1\}$ we then define the regularized vector field $a_{h}$ by

$$
a_{h}(x, t, w) \equiv \int_{B_{1}} a(x+|h| y, t, w) \phi(y) d y=\frac{1}{|h|^{n}} \int_{B_{|h|}(x)} a(y, \tau, w) \phi\left(\frac{y-x}{|h|}\right) d y,
$$

where $z=(x, t) \in Q_{2 \varrho-|h|}^{0}$ and $w \in \mathbb{R}^{N n}$. Then, the vector field $a_{h}$ satisfies the properties (4.2) $1-(4.2)_{6}$.

\subsubsection{A comparison estimate}

Here we shall state the energy and comparison estimate for the initial boundary situation. We still let $u, z_{0}=\left(x_{0}, 0\right)$ and $Q_{\varrho}^{0}\left(z_{0}\right)$ be as above and define $u_{h} \in L^{2}\left(\Lambda_{\varrho^{2}}^{0} ; W^{1,2}\left(B_{\varrho}\left(x_{0}\right) ; \mathbb{R}^{N}\right)\right)$ to be the unique solution of the following Cauchy-Dirichlet problem

$$
\begin{cases}\partial_{t} u_{h}-\operatorname{div} a_{h}\left(x, t, D u_{h}\right)=0 & \text { in } Q_{\varrho}^{0}\left(z_{0}\right), \\ u_{h}=u & \text { on } \partial_{\mathcal{P}} Q_{\varrho}^{0}\left(z_{0}\right),\end{cases}
$$

where $\left\{a_{h}\right\}, 0<|h| \leqslant \min \{\varrho, 1\}$, denotes the family of vector fields constructed in (5.18). Then, the following energy estimate holds

$$
\int_{Q_{\varrho}^{0}\left(z_{0}\right)}\left|D u_{h}\right|^{2} d z \leqslant c(n, L / v) \int_{Q_{\varrho}^{0}\left(z_{0}\right)}\left(1+|D u|^{2}\right) d z .
$$

Moreover, we have the following comparison estimate

$$
\int_{Q_{\varrho}^{0}\left(z_{0}\right)}\left|D u_{h}-D u\right|^{2} d z \leqslant c(n, L / v) \tilde{\theta}^{2}(|h|) \int_{Q_{\varrho}^{0}\left(z_{0}\right)}\left(1+|D u|^{2}\right) d z .
$$

The proof is exactly the same as the one for the lateral boundary situation provided in Section 5.1.2 and therefore we shall not repeat it. We only have to replace $Q_{R}^{+}$by $Q_{\varrho}^{0}\left(z_{0}\right)$. Note also that we did not use the assumption $(5.4)_{7}$ in Section 5.1.2 which is not available now.

\subsubsection{Fractional estimates}

The next lemma provides the higher fractional differentiability of $D u$ up to the initial boundary with respect to the spatial directions and the time direction.

Lemma 5.4. Suppose that $u \in L^{2}\left(0, T ; W^{1,2}\left(\Omega ; \mathbb{R}^{N}\right)\right)$ with $u(\cdot, 0)=0$ on $\Omega$ is a weak solution of (2.3) where the structure conditions $(1.2)_{1}-(1.2)_{3}$ are in force and let $t_{1} \in(0, T)$ and $\tilde{\Omega} \Subset \Omega$. Then, we have

$$
\int_{0}^{t_{1}} \int_{\tilde{\Omega}} \int_{\tilde{\Omega}} \frac{|D u(x, t)-D u(y, t)|^{2}}{|x-y|^{n+2 s}} d x d y d t<\infty, \quad \forall s \in(0, \beta),
$$

and

$$
\int_{\tilde{\Omega}} \int_{0}^{t_{1}} \int_{0}^{t_{1}} \frac{|D u(x, \tau)-D u(x, \tilde{\tau})|^{2}}{|\tilde{\tau}-\tilde{\tau}|^{1+2 s}} d \tau d \tilde{\tau} d x<\infty, \quad \forall s \in\left(0, \frac{\beta}{2}\right) .
$$

Proof. As in the proofs of Lemmas 5.1 and 5.2 we will derive an estimate for the integral of the finite differences $\tau_{h}^{\alpha}[D u], \alpha=1, \ldots, n$ and $\tau_{h}[D u]$, in terms of the increment $|h|$. For this we fix $z_{0} \in \Omega_{0}$ and $Q_{2 \varrho}^{0}\left(z_{0}\right) \subset \Omega_{T}$ with $\varrho \leqslant 1$ and $0<|h| \leqslant \varrho / 4$, where we take $h>0$ when considering finite differences in time. By 


$$
u_{h} \in L^{2}\left(\Lambda_{\varrho^{2}}^{0} ; W^{1,2}\left(B_{\varrho}\left(x_{0}\right) ; \mathbb{R}^{N}\right)\right)
$$

we denote the unique solution of the Cauchy-Dirichlet problem (5.19) in $Q_{\varrho}^{0}\left(z_{0}\right)$. We note that due to (5.4) 1 , $(5.4)_{2},(5.4)_{6}$ and the assumption $\tilde{\theta}(|h|) \leqslant|h|^{\beta}$, the vector fields $a_{h}$ are of the type of the vector fields considered in (4.1) of Section 4.2 (note that we did not assume (4.2) 4 there). In particular, the assumption (4.2) 3 is satisfied with

$$
\gamma=\gamma(h)=c(n) \frac{\tilde{\theta}(|h|)}{|h|} \leqslant c(n)|h|^{\beta-1} .
$$

Therefore, the a priori estimate from Proposition 4.18 with $\sigma=2$ applies to $u_{h}$ such that we have $D^{2} u_{h} \in$ $L^{2}\left(Q_{\varrho / 2}^{0}\left(z_{0}\right) ; \mathbb{R}^{N n^{2}}\right)$ with the estimate

$$
\int_{Q_{\varrho / 2}^{0}\left(z_{0}\right)}\left|D^{2} u_{h}\right|^{2} d z \leqslant c\left(\varrho^{-2}+\gamma^{2}\right) \int_{Q_{\varrho}^{0}\left(z_{0}\right)}\left(1+\left|D u_{h}\right|^{2}\right) d z \leqslant c\left(\varrho^{-2}+|h|^{2 \beta-2}\right) \int_{Q_{\varrho}^{0}\left(z_{0}\right)}\left(1+|D u|^{2}\right) d z .
$$

In the last line we have used the energy estimate (5.20). Note that $c=c(n, N, L / v)$. With the help of (2.7), this estimate yields for the finite differences $\tau_{h}^{\alpha}[D u], \alpha=1, \ldots, n$, that

$$
\int_{Q_{\varrho / 4}^{0}\left(z_{0}\right)}\left|\tau_{h}^{\alpha}\left[D u_{h}\right]\right|^{2} d z \leqslant c(n)|h|^{2} \int_{Q_{\varrho / 2}^{0}\left(z_{0}\right)}\left|D^{2} u_{h}\right|^{2} d z \leqslant c|h|^{2 \beta} \varrho^{-2} \int_{Q_{\varrho}^{0}\left(z_{0}\right)}\left(1+|D u|^{2}\right) d z .
$$

Now, we employ the comparison estimate (5.21) in completely the same way as we did in the proof of Lemma 5.1 (with $\vartheta=0$ ) in order to derive a similar estimate for $\tau_{h}^{\alpha}[D u]$. Proceeding this way we end up with

$$
\int_{Q_{\varrho / 4}^{0}\left(z_{0}\right)}\left|\tau_{h}^{\alpha}[D u]\right|^{2} d z \leqslant c|h|^{2 \beta} \varrho^{-2} \int_{Q_{\varrho}^{0}\left(z_{0}\right)}\left(1+|D u|^{2}\right) d z
$$

where $c=c(n, N, L / v)$. At this stage we note that the preceding estimate holds true for interior cylinders as well (see [16, Lemma 9.4]). An application of Lemma 2.5(ii) now yields the assertion concerning the spatial fractional differentiability.

We now come to the time-direction. We let $z_{0}, \varrho, h, a_{h}, \gamma$ and $u_{h}$ still be as above. We consider $0<\lambda \leqslant(\varrho / 2)^{2}$ and set $h=\lambda^{\frac{1}{2}}$. Applying Proposition 4.20 to $u_{h}$ we infer

$$
\int_{Q_{\varrho / 2}^{0}}\left|\tau_{\lambda}\left[D u_{h}\right]\right|^{2} d z \leqslant c \lambda\left(\varrho^{-2}+\gamma^{2}\right) \int_{Q_{\varrho}^{0}}\left(1+\left|D u_{h}\right|^{2}\right) d z \leqslant c \lambda^{\beta} \varrho^{-2} \int_{Q_{\varrho}^{0}}\left(1+|D u|^{2}\right) d z,
$$

where we have also used the energy estimate (5.20) and $c=c(n, N, L / v)$. To derive a similar estimate for $\tau_{\lambda}[D u]$ we proceed as in the proof of Lemma 5.2, i.e. we use the comparison estimate (5.21) in order to pass over the preceding estimate to $\tau_{\lambda}[D u]$. This leads us to

$$
\int_{Q_{\varrho / 2}^{0}\left(z_{0}\right)}\left|\tau_{\lambda}[D u]\right|^{2} d z \leqslant c \lambda^{\beta} \varrho^{-2} \int_{Q_{\varrho}^{0}\left(z_{0}\right)}\left(1+|D u|^{2}\right) d z,
$$

where $c=c(n, N, L / \nu)$. We note that the preceding estimate also holds in the interior situation (see [16, Lemma 9.5]). Lemma 2.5(i) now yields the fractional differentiability of $D u$ with respect to time and this finishes the proof of the lemma.

Finally, we briefly summarize, what we have achieved up to now. Combining the fractional differentiability with respect to space and time from Lemma 5.4, we arrive at the following fractional differentiability result: 
Theorem 5.5. Let $u \in L^{2}\left(0, T ; W^{1,2}\left(\Omega ; \mathbb{R}^{N}\right)\right)$ be a weak solution of the non-linear parabolic system (1.1) under the assumptions $(1.2)_{1}-(1.2)_{3}$ and $(1.4)_{2}$ where $u=g$ on the initial boundary $\Omega_{0}$. Then for any $\tilde{\Omega} \subset \Omega, t_{1} \in(0, T)$ and $s \in(0, \beta)$ there holds

$$
D u \in W^{s, \frac{s}{2}, 2}\left(\tilde{\Omega} \times\left(0, t_{1}\right) ; \mathbb{R}^{N n}\right), \quad \forall s<\beta .
$$

\subsubsection{Proof of Theorem 1.5}

At this stage, we mention that - contrary to the lateral boundary situation - Theorem 5.5, stating that $D u \in W^{s, s / 2,2}$ for any $s \in(0, \beta)$, does not immediately imply the existence of at least one regular initial boundary point. Indeed, the application of Lemma 2.6 yields $\operatorname{dim}_{\mathcal{P}}\left(\Omega_{0} \cap \Sigma\right)<n+2-2 \beta$. Since $\beta \in(0,1)$ this quantity is still larger than $n$; note that the parabolic Hausdorff-dimension of the initial boundary $\Omega_{0}$ is $n$. Hence, in order to prove the existence of regular initial boundary points we need to establish a higher integrability improvement of Theorem 5.5, i.e. that $D u \in W^{s, s / 2, \sigma}$ for some $\sigma>2$ independent of $\beta$. This will be the main effort in the

Proof of Theorem 1.5. Our aim is to show that

$$
\operatorname{dim}_{\mathcal{P}}\left(\Omega_{0} \backslash \operatorname{Reg}_{\mathcal{P}} u\right)<n
$$

which implies that $\mathcal{H}_{\mathcal{P}}^{n}$-almost every initial boundary point is a regular point of $D u$. By the transformation explained in Section 2.1 we can assume that we have zero initial boundary values. Now, we consider a fixed cylinder $Q_{\varrho}^{0}\left(z_{0}\right) \subset \Omega_{T}$ with $z_{0} \in \Omega_{0}$. From the characterization of regular boundary points in Theorem 1.2 we already know that $D_{\varrho}\left(z_{0}\right) \backslash$ $\operatorname{Reg}_{\mathcal{P}} u \subset D_{\varrho}\left(z_{0}\right) \cap\left(\Sigma^{1} \cup \Sigma^{2}\right)$. In the following we will show that $D u \in W^{s, s / 2, \sigma}\left(Q_{\varrho / 16}^{0} ; \mathbb{R}^{N n}\right)$ for some $\sigma>2$ by improving the comparison estimate (5.21) and the fractional differentiability properties of the comparison function $u_{h}$ (from the proof of Lemma 5.4).

Hence, our aim is to determine $\delta>0$ such that $\beta>1-\delta$ implies that

$$
\operatorname{dim}_{\mathcal{P}}\left(D_{\varrho / 16}\left(z_{0}\right) \cap \Sigma\right)<n .
$$

By an up to the initial boundary higher integrability result as for instance provided by [8,31] we infer the existence of $\sigma_{1}=\sigma_{1}(n, N, L / v)>2$ such that

$$
D u \in L^{\sigma_{1}}\left(Q_{\varrho}^{0}\left(z_{0}\right) ; \mathbb{R}^{N n}\right) .
$$

Now, we denote by $\left\{a_{h}\right\}$ the vector fields constructed in Section 5.2.1 and let $u_{h} \in L^{2}\left(\Lambda_{\varrho^{2}}^{0} ; W^{1,2}\left(B_{\varrho}\left(x_{0}\right) ; \mathbb{R}^{N}\right)\right)$ be the unique solution of the initial Cauchy-Dirichlet problem (5.19) in $Q_{\varrho}^{0}\left(z_{0}\right)$. Taking the difference of the systems (5.17) and (5.19) we see (as in the proof of Theorem 1.3) that $u_{h}-u$ is a weak solution of the following linear initial Cauchy-Dirichlet problem:

$$
\begin{cases}\partial_{t}\left(u_{h}-u\right)-\operatorname{div}\left(\tilde{\mathcal{A}}(h) D\left(u_{h}-u\right)\right)=\operatorname{div}\left(a_{h}(\cdot, D u)-a(\cdot, D u)\right) & \text { in } Q_{\varrho}^{0}\left(z_{0}\right), \\ u_{h}-u=0 & \text { on } \partial_{\mathcal{P}} Q_{\varrho}^{0}\left(z_{0}\right),\end{cases}
$$

where $\tilde{\mathcal{A}}(h)(z)=\int_{0}^{1} \partial_{w} a_{h}\left(z, D u+s\left(D u_{h}-D u\right)\right) d s$ are the coefficients defined in (5.11). From our assumptions $(5.4)_{1}$ and (5.4) 2 we know that $\langle\tilde{\mathcal{A}}(h)(z) w, w\rangle \geqslant v|w|^{2}$ and also $|\tilde{\mathcal{A}}(h)(z)| \leqslant L$ for any choice of $w \in \mathbb{R}^{N n}$ and $z \in Q_{\varrho}^{0}\left(z_{0}\right)$. Therefore, we can once again use the up to the initial boundary higher integrability from [8,31] (note that the right-hand side is $b(z) \equiv a_{h}(z, D u)-a(z, D u) \in L^{\sigma_{1}}\left(Q_{\varrho}^{0}\left(z_{0}\right) ; \mathbb{R}^{N n}\right)$; see (5.4) $1,(1.2)_{1}$ and (5.24)) to infer the existence of $\sigma_{2}=\sigma_{2}(n, N, L / v) \in\left(2, \sigma_{1}\right]$ such that $D u_{h}-D u \in L^{\sigma_{2}}\left(Q_{\varrho / 2}^{0}\left(z_{0}\right) ; \mathbb{R}^{N n}\right)$. Moreover, for any choice of $\sigma \in\left(2, \sigma_{2}\right]$ holds

$$
\int_{Q_{\varrho / 2}^{0}\left(z_{0}\right)}\left|D u_{h}-D u\right|^{\sigma} d z \leqslant c\left(\int_{Q_{\varrho}^{0}\left(z_{0}\right)}\left|D u_{h}-D u\right|^{2} d z\right)^{\frac{\sigma}{2}}+c \int_{Q_{\varrho}^{0}\left(z_{0}\right)}\left|a_{h}(z, D u)-a(z, D u)\right|^{\sigma} d z,
$$

where $c=c(n, N, L / v)$. In the following we shall estimate the integrals appearing on the right-hand side of the preceding inequality. For the first one we use the comparison estimate (5.21) and Hölder's inequality to infer 


$$
\left(\int_{Q_{\varrho}^{0}\left(z_{0}\right)}\left|D u_{h}-D u\right|^{2} d z\right)^{\frac{\sigma}{2}} \leqslant c(n, L / v)|h|^{\beta \sigma} f_{Q_{\varrho}^{0}\left(z_{0}\right)}\left(1+|D u|^{\sigma}\right) d z .
$$

The second integral can be treated using the pointwise estimate $(5.4)_{5}$ to obtain

$$
\int_{Q_{\varrho}^{0}\left(z_{0}\right)}\left|a_{h}(\cdot, D u)-a(\cdot, D u)\right|^{\sigma} d z \leqslant c(n, L / \nu)|h|^{\beta \sigma} \int_{Q_{\varrho}^{0}\left(z_{0}\right)}\left(1+|D u|^{\sigma}\right) d z .
$$

Inserting this above we arrive at

$$
\int_{Q_{\varrho / 2}^{0}\left(z_{0}\right)}\left|D u_{h}-D u\right|^{\sigma} d z \leqslant c(n, N, L / v)|h|^{\beta \sigma} \int_{Q_{\varrho}^{0}\left(z_{0}\right)}\left(1+|D u|^{\sigma}\right) d z,
$$

which is the desired improvement of the comparison estimate (5.21) we were looking for. We note that the last inequality implies the energy bound:

$$
\int_{Q_{\varrho / 2}^{0}\left(z_{0}\right)}\left|D u_{h}\right|^{\sigma} d z \leqslant c(n, L / v) \int_{Q_{\varrho}^{0}\left(z_{0}\right)}\left(1+|D u|^{\sigma}\right) d z .
$$

Next, we are going to improve the $W^{s, \frac{s}{2}, 2}$-estimate from Theorem 5.5, utilizing the higher integrability of $D^{2} u_{h}$. We first fix $s \in(0, \beta)$. Applying Proposition 4.18, we infer the existence of $\sigma_{3}=\sigma_{3}(n, N, L / v) \in\left(2, \sigma_{2}\right]$, such that $\left|D^{2} u_{h}\right| \in L^{\sigma_{3}}\left(Q_{\varrho / 2}^{0}\left(z_{0}\right)\right)$. Moreover, for any choice of $\sigma \in\left(2, \sigma_{3}\right]$ we have

$$
\int_{Q_{\varrho / 4}^{0}\left(z_{0}\right)}\left|D^{2} u_{h}\right|^{\sigma} d z \leqslant c\left(\varrho^{-\sigma}+\gamma^{\sigma}\right) \int_{Q_{\varrho / 2}^{0}\left(z_{0}\right)}\left(1+\left|D u_{h}\right|^{\sigma}\right) d z \leqslant c|h|^{\sigma(\beta-1)} \varrho^{-\sigma} \int_{Q_{\varrho}^{0}\left(z_{0}\right)}\left(1+|D u|^{\sigma}\right) d z
$$

where we have used in the last line (5.26) and $\gamma=c(n)|h|^{1-\beta}$ from (5.22). Note that $c=c(n, N, L / v)$. This yields for $\alpha=1, \ldots, n$ and $|h| \leqslant \varrho / 8$ that

$$
\int_{Q_{\varrho / 8}^{0}\left(z_{0}\right)}\left|\tau_{h}^{\alpha}\left[D u_{h}\right]\right|^{\sigma} d z \leqslant c|h|^{\sigma \beta} \varrho^{-\sigma} \int_{Q_{\varrho}^{0}\left(z_{0}\right)}\left(1+|D u|^{\sigma}\right) d z .
$$

This estimate can now be carried over to $D u$ via (5.25) by the comparison argument we used several times before (see for instance the proof of Theorem 1.3). This leads us to the following improvement of (5.23):

$$
\int_{Q_{\varrho / 8}^{0}\left(z_{0}\right)}\left|\tau_{h}^{\alpha}[D u]\right|^{\sigma} d z \leqslant c|h|^{\sigma \beta} \varrho^{-\sigma} \int_{Q_{\varrho}^{0}\left(z_{0}\right)}\left(1+|D u|^{\sigma}\right) d z .
$$

Finally, we consider the time-direction. We will attain an estimate for the $L^{\sigma}$-norm of $\left|\tau_{\lambda}[D u]\right|$ with the help of the comparison map $u_{h}$ with the choice $h=\lambda^{\frac{1}{2}}$ and $0<\lambda \leqslant(\varrho / 4)^{2}$. From Proposition 4.20 and Hölder's inequality we infer the existence of $\sigma_{4}=\sigma_{4}(n, N, L / v) \in\left(2, \sigma_{3}\right]$ such that for any $\sigma \in\left(2, \sigma_{4}\right]$ there holds

$$
\int_{Q_{\varrho / 4}^{0}\left(z_{0}\right)}\left|\tau_{\lambda}\left[D u_{h}\right]\right|^{\sigma} d z \leqslant c \lambda^{\frac{\sigma}{2}}\left(\varrho^{-\sigma}+\gamma^{\sigma}\right) \int_{Q_{\varrho / 2}^{0}\left(z_{0}\right)}\left(1+\left|D u_{h}\right|^{\sigma}\right) d z \leqslant c \lambda^{\frac{\sigma}{2} \beta} \varrho^{-\sigma} \int_{Q_{\varrho}^{0}\left(z_{0}\right)}\left(1+|D u|^{\sigma}\right) d z,
$$

where in the last line we have used (5.26) and $\gamma=c(n) h^{\beta-1}=c(n) \lambda^{\frac{\beta-1}{2}}$. This estimate is carried over to $D u$ by joining (5.25) with the preceding estimate (see for instance the proof of Theorem 1.3). We obtain

$$
\int_{Q_{\varrho / 8}^{0}\left(z_{0}\right)}\left|\tau_{\lambda}[D u]\right|^{\sigma} d z \leqslant c|\lambda|^{\frac{\sigma}{2} \beta} \varrho^{-\sigma} \int_{Q_{\varrho}^{0}\left(z_{0}\right)}\left(1+|D u|^{\sigma}\right) d z
$$


where $c=c(n, N, L / v)$. Combining (5.28) and (5.29), we infer from Lemma 2.5 that $D u \in W^{s, s / 2, \sigma}\left(Q_{\varrho / 16}^{0}\left(z_{0}\right) ; \mathbb{R}^{N n}\right)$. Since $s \in(0, \beta)$ was arbitrary we conclude that $D u \in W^{s, s / 2, \sigma}\left(Q_{\varrho / 16}^{0}\left(z_{0}\right) ; \mathbb{R}^{N n}\right)$ for any $s \in(0, \beta)$ and $\sigma \in\left(2, \sigma_{4}\right]$. At this stage we perform the choice of the constants. We first choose $\sigma=\sigma_{4}=\sigma_{4}(n, N, L / v)$. Then, $\sigma=\sigma(n, N, L / v)$ does not depend on $\beta$. Next, we choose $\delta$ in (1.9) such that

$$
\delta \equiv \frac{1}{2}-\frac{1}{\sigma}>0
$$

Note here that the dependencies of $\delta$ from the structural parameters are the same as for $\sigma$. Since $\beta>1-\delta$ we can choose $s \in(1-\delta, \beta)$. Applying Lemma 2.6(i) we infer that $\operatorname{dim}_{\mathcal{P}}\left(D_{\varrho / 16}\left(z_{0}\right)\right) \cap \Sigma<n+2-s \sigma<n$. The last inequality follows from the fact that $s \sigma>(1-\delta) \sigma=\sigma / 2+1>2$. Since $z_{0}$ was an arbitrary point on the initial boundary $\Omega_{0}$ the assertion of Theorem 1.5 follows by a standard covering argument.

\section{Acknowledgements}

This work is supported by the ERC grant 207573 "Vectorial Problems".

\section{References}

[1] E. Acerbi, G. Mingione, Gradient estimates for a class of parabolic systems, Duke Math. J. 136 (2007) 285-320.

[2] E. Acerbi, G. Mingione, G.A. Seregin, Regularity results for parabolic systems related to a class of non-newtonian fluids, Ann. Inst. H. Poincaré Anal. Non Linéaire 21 (1) (2004) 25-60.

[3] A.A. Arkhipova, On a partial regularity up to the boundary of weak solutions to quasilinear parabolic systems with quadratic growth, Zap. Nauchn. Sem. S.-Peterburg. Otdel. Mat. Inst. Steklov. (POMI) 249 (5) (1997) 20-39.

[4] L. Beck, Partial regularity for weak solutions of nonlinear elliptic systems: The subquadratic case, Manuscripta Math. 123 (4) (2007) $453-491$.

[5] C. Bennett, R. Sharpley, Interpolation of Operators, Academic Press, Boston, 1988.

[6] V. Bögelein, Partial regularity and singular sets of solutions of higher order parabolic systems, Ann. Mat. Pura Appl. 188 (2009) 61-122.

[7] V. Bögelein, F. Duzaar, G. Mingione, The boundary regularity of non-linear parabolic systems I, Ann. Inst. H. Poincaré Anal. Non Linéaire 27 (1) (2010) 201-255 (in this issue).

[8] V. Bögelein, M. Parviainen, Self-improving property of nonlinear higher order parabolic systems near the boundary, NoDEA Nonlinear Differential Equations Appl., doi:10.1007/s00030-009-0038-5.

[9] B. Bojarski, T. Iwaniec, Analytical foundations of the theory of quasiconformal mappings in $\mathbb{R}^{n}$, Ann. Acad. Sci. Fenn. Ser. A I 8 (1983) 257-324.

[10] E. DiBenedetto, Degenerate Parabolic Equations, Universitext, Springer-Verlag, New York, 1993.

[11] Y.Z. Chen, E. DiBenedetto, Boundary estimates for solutions of nonlinear degenerate parabolic systems, J. Reine Angew. Math. 395 (1989) $102-131$.

[12] A. Domokos, Differentiability of solutions for the non-degenerate $p$-Laplacian in the Heisenberg group, J. Differential Equations 204 (2004) 439-470.

[13] F. Duzaar, J.F. Grotowski, Optimal interior partial regularity for nonlinear elliptic systems: The method of $a$-harmonic approximation, Manuscripta Math. 103 (2000) 267-298.

[14] F. Duzaar, J.F. Grotowski, M. Kronz, Partial and full boundary regularity for minimizers of functionals with nonquadratic growth, J. Convex Anal. 11 (2004) 437-476.

[15] F. Duzaar, J. Kristensen, G. Mingione, The existence of regular boundary points for non-linear elliptic systems, J. Reine Angew. Math. (Crelles J.) 602 (2007) 17-58.

[16] F. Duzaar, G. Mingione, Second order parabolic systems, optimal regularity, and singular sets of solutions, Ann. Inst. H. Poincaré Anal. Non Linéaire 22 (2005) 705-751.

[17] F. Duzaar, G. Mingione, Harmonic type approximation lemmas, J. Math. Anal. Appl. 352 (2009) 301-335.

[18] F. Duzaar, G. Mingione, K. Steffen, Parabolic systems with polynomial growth and regularity, Mem. Amer. Math. Soc., in press.

[19] F.G. Duzaar, K. Steffen, Optimal interior and boundary regularity for almost minimizers to elliptic variational integrals, J. Reine Angew. Math. 546 (2002).

[20] C. Fefferman, E.M. Stein, $\mathrm{H}^{p}$ spaces of several variables, Acta Math. 129 (1972) 137-193.

[21] M. Giaquinta, A counter-example to the boundary regularity of solutions to quasilinear systems, Manuscripta Math. 24 (1978) 217-220.

[22] M. Giaquinta, Multiple Integrals in the Calculus of Variations and Nonlinear Elliptic Systems, Princeton Univ. Press, Princeton, NJ, 1983.

[23] E. Giusti, Direct Methods in the Calculus of Variations, World Scientific Publishing Company, Singapore, 2003.

[24] T. Iwaniec, On $L^{p}$-integrability in PDE's and quasiregular mappings for large exponents, Ann. Acad. Sci. Fenn. Ser. A I 7 (2) (1982) $301-322$.

[25] J. Kristensen, G. Mingione, The singular set of minima of integral functionals, Arch. Ration. Mech. Anal. 180 (2006) 331-398.

[26] J. Kristensen, G. Mingione, Boundary regularity in variational problems, in press.

[27] J. Kristensen, G. Mingione, Boundary regularity of minima, Rend. Lincei Mat. Appl. 19 (2008) 265-277.

[28] G. Mingione, The singular set of solutions to non-differentiable elliptic systems, Arch. Ration. Mech. Anal. 166 (2003) $287-301$. 
[29] G. Mingione, Bounds for the singular set of solutions to non linear elliptic systems, Calc. Var. Partial Differential Equations 18 (2003) $373-$ 400.

[30] G. Mingione, Regularity of minima: An invitation to the dark side of the calculus of variations, Appl. Math. 51 (2006) 355-425.

[31] M. Parviainen, Global gradient estimates for degenerate parabolic equations in nonsmooth domains, Ann. Mat. Pura Appl. 188 (2) (2009) 333-358.

[32] J. Stará, O. John, J. Malý, Counterexamples to the regularity of weak solutions of the quasilinear parabolic system, Comment. Math. Univ. Carolin. 27 (1986) 123-136.

[33] E.W. Stredulinsky, Higher integrability from reverse Hölder inequalities, Indiana Univ. Math. J. 29 (1980) $407-413$.

[34] A. Zygmund, Trigonometric Series I, Cambridge Univ. Press, Cambridge, 1977. 